TRANSACTIONS OF THE

AMERICAN MATHEMATICAL SOCIETY

Volume 348, Number 9, September 1996

\title{
PACKAGE DEAL THEOREMS AND SPLITTING ORDERS IN DIMENSION 1
}

\author{
LAWRENCE S. LEVY AND CHARLES J. ODENTHAL
}

\begin{abstract}
Let $\Lambda$ be a module-finite algebra over a commutative noetherian ring $R$ of Krull dimension 1. We determine when a collection of finitely generated modules over the localizations $\Lambda_{\mathbf{m}}$, at maximal ideals of $R$, is the family of all localizations $M_{\mathbf{m}}$ of a finitely generated $\Lambda$-module $M$. When $R$ is semilocal we also determine which finitely generated modules over the $J(R)$-adic completion of $\Lambda$ are completions of finitely generated $\Lambda$-modules.

If $\Lambda$ is an $R$-order in a semisimple artinian ring, but not contained in a maximal such order, several of the basic tools of integral representation theory behave differently than in the classical situation. The theme of this paper is to develop ways of dealing with this, as in the case of localizations and completions mentioned above. In addition, we introduce a type of order called a "splitting order" of $\Lambda$ that can replace maximal orders in many situations in which maximal orders do not exist.
\end{abstract}

\section{INTRODUCTION}

The original motivation for this paper comes from the companion paper [LO] in which the authors determine which $R$-orders $\Lambda$ in semisimple artinian rings satisfy the Krull-Schmidt theorem for f.g. (finitely generated) modules, and which such $\Lambda$ satisfy the Krull-Schmidt theorem for f.g. torsionfree modules. Since we do not assume that $\Lambda$ is contained in a maximal order, it is necessary to rebuild a number of the basic tools of integral representation theory, finding answers to the questions mentioned in the abstract of this paper. We hope that these tools have some interest in their own right, and we therefore go beyond the requirements of [LO] in a number of places. In any event, clarity of exposition seems to require separating the general tools studied here from those directly related to the Krull-Schmidt problem.

Notation 1.1. The following notation will remain in effect throughout this paper. $R$ denotes a commutative noetherian ring of Krull dimension 1, and $\Lambda$ denotes a module-finite $R$-algebra. $Q=Q(R)$ denotes the (finite) set of minimal prime ideals of $R$, and $R_{Q}$ and $\Lambda_{Q}$ denote the localizations obtained by inverting the elements of $R-\bigcup Q$.

Since $R_{Q}$ is an artinian ring, so is $\Lambda_{Q}$. When $\Lambda$ has no artinian ring direct summands (e.g. in integral representation theory) we think of $\Lambda_{Q}$ as the natural

Received by the editors April 11, 1994 and, in revised form, September 25, 1995.

1991 Mathematics Subject Classification. Primary 16P40, 16P50, 16W60; Secondary 13E05, 13B30, 13J10.

Key words and phrases. Localization, completion, normalization, splitting order, package deal. Levy's research was partially supported by NSF and NSA grants.

(C)1996 American Mathematical Society 
artinian quotient ring of $\Lambda$, because $\Lambda_{Q}=\Lambda_{Q(R)}$ is then independent of the particular ring $R$ over which $\Lambda$ is a module-finite algebra. See Lemma 1.5 and Remark 1.2. Note that the natural map $\Lambda \rightarrow \Lambda_{Q}$ is not necessarily one-to-one. The purpose of $\Lambda_{Q}$ is the same as that of the quotient field of an integral domain: to establish a link, via fraction-formation, between rings of dimension 0 and dimension 1 . The fact that the natural map $\Lambda \rightarrow \Lambda_{Q}$ can fail to be one-to-one is no disadvantage in this regard.

The $\Lambda$-modules that really interest us, in this paper, are always f.g. But we have to look at non-f.g. modules often enough that (unlike in [LO]) we say f.g. when we mean it.

We say that an $R$-module $M$ is $Q$-torsionfree if the elements of $R-\cup Q$ act as non-zero-divisors on $M$, that is, if the natural map $M \rightarrow M_{Q}$ is one-to-one. Some care is required in dealing with this notion, since $R$ itself can fail to be $Q$-torsionfree. This may seem disconcerting at first. But we show that, for any f.g. $R$-module $M$, $M_{\mathbf{m}}$ is $Q$-torsionfree for almost all maximal ideals $\mathbf{m}$ of $R$, a simple fact that turns out to be very useful (see Section 2).

When $R$ has no nilpotent elements, $R$ itself becomes a $Q$-torsionfree $R$-module. In this situation we relax the terminology, and use the more common term "torsionfree module". Whenever we refer to a torsionfree or $Q$-torsionfree $\Lambda$-module $M$, we always mean that $M$ is $Q$-torsionfree as an $R$-module. When $\Lambda$ has no artinian ring direct summands this property of $M$ is independent of the ring $R$ over which $\Lambda$ is a module-finite algebra, because $R_{Q} \otimes_{R} M \cong \Lambda_{Q} \otimes_{\Lambda} M$ and $\Lambda_{Q}$ is independent of $R$.

(1.1.1) We call $\Lambda$ an $R$-order in the semisimple artinian ring $\Lambda_{Q}$ if (in addition to the properties of $R$ and $\Lambda$ mentioned in the first paragraph of Notation 1.1) $R$ has no nilpotent elements $\neq 0$, the canonical map $\Lambda \rightarrow \Lambda_{Q}$ is a monomorphism and the ring $\Lambda_{Q}$ is semisimple artinian.

The hypothesis about no nilpotent elements is no loss of generality, because any nilpotent element of $R \cdot 1_{\Lambda}$ generates a nilpotent ideal of $\Lambda_{Q}$ and therefore equals zero.

Here is a summary of the problems considered in this paper.

- Section 2: Packages of Localizations. Consider a family $\{M(\mathbf{m}) \mid \mathbf{m}$ is a maximal ideal of $R\}$ where each $M(\mathbf{m})$ is a f.g. $\Lambda_{\mathbf{m}}$-module. For which such families is there a f.g. $\Lambda$-module $M$ such that $M_{\mathbf{m}} \cong M(\mathbf{m})$ for every $\mathbf{m}$ ?

When $\Lambda=R$ and is an integral domain the necessary and sufficient conditions are especially easy to state: (i) Almost all (i.e. all but finitely many) of the $M(\mathbf{m})$ must be free $R_{\mathbf{m}}$-modules; and (ii) All of the $R_{Q}$-vector spaces $R_{Q} \otimes_{R} M(\mathbf{m})=M(\mathbf{m})_{Q}$ must have the same dimension. [Recall that when $R$ is an integral domain $R_{Q}$ is the field of quotients of $R$.]

In order to state the general result, it is easiest to first specify the f.g. $\Lambda_{Q}$-module $X$ that will be isomorphic to the $Q$-localization of the $M$ that we wish to build. As a first approximation to $M$, let $N$ be any f.g. $\Lambda$-moldule such that $N_{Q} \cong X$. (For example, let $N$ be the $\Lambda$-module generated by some finite set of $\Lambda_{Q}$-generators of $X$.) We prove that it is possible to change the isomorphism classes of any finite set of localizations $N_{\mathbf{m}}$ arbitrarily provided that we do not change $\left(N_{\mathbf{m}}\right)_{Q}$ (hence do not change $X=N_{Q}$ ). The resulting $\Lambda$-modules $M$ yield the most general collection of isomorphism classes $\left\{M_{\mathbf{m}}\right\}$ such that $M_{Q} \cong X$. The basic restriction that governs this whole section is that, although we can change any finite number 
of isomorphism classes of localizations arbitrarily (provided that we do not change their $Q$-localization), we can never change infinitely many of them.

To clarify the spirit of this complicated result, we show how it applies to the special case previously discussed: $R=\Lambda$, an integral domain. Condition (ii) is obviously necessary for the existence of $M$ because $\left(M_{\mathbf{m}}\right)_{Q}=M_{Q}$ in this special situation. Conversely, suppose that conditions (i) and (ii) hold. Then, by condition (ii), the $\Lambda_{Q}$-module $X=M(\mathbf{m})_{Q}$ is independent of our choice of $\mathbf{m}$, and provides the $X$ needed to begin the construction in the general theorem. Let $n$ be the dimension of this $\Lambda_{Q}$-vector space $X$. Then for our first approximation to the desired $M$ we choose $N=\Lambda^{n}$. Almost every $M(\mathbf{m})$ is $\Lambda_{\mathbf{m}}$-free, by condition (i), and its rank is $n$ by condition (ii). Thus we have $N_{\mathbf{m}} \cong M(\mathbf{m})$ for almost all $\mathbf{m}$. Our ability to arbitrarily change any finite number of localizations of $N$ enables us to correct the finitely many remainimg localizations, and complete the proof that $M$ exists. Moreover, the fact that we can never change infinitely many localizations (without changing $M_{Q}=X$ ) shows that condition (i) is also a necessary condition.

But what about the infinitely many isomorphism classes of localizations that we cannot change, when we are not in this special situation? We give a complete answer when $\Lambda$ has no nilpotent ideals. In the commutative case $R=\Lambda$ (reduced, but not necessarily a domain) almost every $\Lambda_{\mathbf{m}}$ must still be free, but the rank need not be the same for all $\mathbf{m}$. For noncommutative semiprime $\Lambda$ we prove that, for almost all $\mathbf{m}$, there is a canonical "default" projective $\Lambda_{\mathbf{m}}$-module that is determined by its $Q$-localization, and this module replaces the free module in condition (i) of the special case $R=\Lambda$.

An application of this is that indecomposability is a semilocal property. That is, if a f.g. $\Lambda$-module $M$ is indecomposable, then there is a finite collection $\mathcal{M}$ of maximal ideals of $R$ such that the localization $M_{\mathcal{M}}$ is an indecomposable $\Lambda_{\mathcal{M}}$-module, and remains indecomposable if $\mathcal{M}$ is enlarged to any larger finite set of maximal ideals. [Here $M_{\mathcal{M}}=\{m / d \mid m \in M$ and $d \in R-\bigcup \mathcal{M}\}$.] If $\Lambda$ is any of the rings studied in integral representation theory and $M$ is a torsionfree $\Lambda$-module, this is well-known and easy to see: $\Lambda_{\mathbf{m}}$ is a maximal order for almost all $\mathbf{m}$, and so we can take $\mathcal{M}$ to be the remaining maximal ideals. This indecomposability property, in our full generality, is one of the key steps in the reduction of the Krull-Schmidt problem to the case that $R$ is semilocal in $[\mathrm{LO}]$.

- Section 3: Completions. Here $R$ is semilocal and $\hat{\Lambda}$ denotes the $J(R)$-adic completion of $\Lambda$. In order to define a f.g. $\Lambda$-module by specifying its completion, one must know which f.g. $\hat{\Lambda}$-modules $X$ are completions of f.g. $\Lambda$-modules. As the first step in answering this question the main result of this section shows that $X$ is the $J(R)$-adic completion of some $\Lambda$-module if and only if $X_{Q}$ is the $J(R)$-induced completion of some $\Lambda_{Q}$-module. The special case in which $\Lambda$ is an $R$-order in a semisimple artinian ring, $\Lambda$ is contained in a maximal order, and $R$ is a discrete valuation ring, is often attributed to Heller [H '61].

When $\Lambda$ is an $R$-order in a semisimple artinian ring, as defined in (1.1.1), one can give a more complete answer to the question of when $X$ is the completion of some $\Lambda$-module than the main result of the present section gives. We do this in Section 6 using the main result of the present section.

A difficulty in the present section is that the $J(R)$-induced completion $\hat{\Lambda}_{Q}=$ $\hat{R} \otimes_{R} \Lambda_{Q}$ of $\Lambda_{Q}$ is not an "adic" completion of a f.g. $R_{Q}$-module, in the sense 
of commutative algebra. For example, if $\Lambda=R$ and is a discrete valuation ring, then $R_{Q}$ is a field; so all elements of the filtration $\left\{J(R)^{n} R_{Q} \mid n=1,2, \ldots\right\}$ equal $R_{Q}$ itself, and hence do not generate a worthwhile topology. Of course, what we are generalizing is the completion of a field with respect to a discrete valuation. It is customary to call this a $p$-adic completion when it arises in integral representation theory, and a generic formal fiber ring when it arises in current commutative algebra. We call it the $J(R)$-induced completion of $\Lambda_{Q}$, and take some space to develop the basic facts we need but could not find elsewhere.

- Section 4: Normalizations. In the noncommutative part of integral representation theory it is customary to work with maximal orders rather than integral closures for two reasons. First is that integral closures in the total quotient ring usually do not exist in a noncommutative setting. The second difficulty is that, even when an integral closure exists (e.g. in the commutative case) it is not necessarily module-finite over the original ring.

Let $\Lambda$ be an $R$-order in the semisimple artinian ring $\Lambda_{Q}$. In order to deal with the difficulties in the preceding paragraph, a normalization was defined in [GL '89] to be a ring $\Gamma$ that is maximal with respect to the properties that $\Lambda \subseteq \Gamma \subseteq \Lambda_{Q}$ and $\Gamma$ is integral over $R$. The present section briefly presents the basic properties of normalizations that will be needed subsequently. In particular, normalizations provide a crucial link between our theory and integral representation theory because $\Gamma$ turns out to be a direct sum of classical maximal orders over Dedekind domains in simple artinian rings. Therefore $\Gamma$ has all of the familiar ring-theoretical properties of maximal orders; for example, every left ideal is a projective $\Gamma$-module.

- Section 5: Splitting Orders. In this section $R$ is semilocal and $\Lambda$ is an $R$ order in the semisimple artinian ring $\Lambda_{Q}$. One of the fundamental tools in integral representation theory is the use of a conductor ideal to relate the module theory over $\Lambda$ to that of a maximal order containing $\Lambda$. Let $\Gamma$ be a normalization of $\Lambda$. If $\Gamma$ is not module-finite over $R$, there is no common "conductor" ideal $C$ such that both rings $\Lambda / C$ and $\Gamma / C$ are artinian. The main result of this section is the existence of what we call a splitting order $\Omega$ between $\Lambda$ and $\Gamma$. This $\Omega$ is large enough so that: (i) no splitting, ramification, or residue-growth (of maximal ideals) occurs between $\Omega$ and $\Gamma$, (ii) $\Omega$ decomposes like $\Gamma$ (i.e. primitive idempotents of $\Omega$ remain primitive in $\Gamma$ and $\Omega d \cong \Omega e \Longleftrightarrow \Gamma d \cong \Gamma e$ for all idempotents $d, e \in \Omega$ ), and (iii) $\Gamma$ is a centralizing extension of $\Omega$, in fact $\Gamma$ is generated as a ring by $\Omega$ and central elements of $\Gamma$. Since $\Omega$ is a f.g. $R$-module, there exist conductor ideals for $\Lambda$ and $\Omega$. In the proof that splitting orders exist (Theorem 5.2) we make use of the lying-over theorem for prime ideals of centralizing extensions of noncommutative noetherian rings.

We prove that splitting orders have the Krull-Schmidt property for f.g. projective modules. In addition, given any finite set $\mathcal{F}$ of torsionfree $\Lambda$-modules, $\Omega$ can be chosen large enough so that $\Omega M$ is $\Omega$-projective for every $M \in \mathcal{F}$.

- Section 6: Completions of Orders, Artinian Pairs. In this section $R$ is semilocal and $\Lambda$ is an $R$-order in the semisimple artinian ring $\Lambda_{Q}$. Let $X$ be a f.g. module over the $J(R)$-adic completion $\hat{\Lambda}$ of $\Lambda$. We return to the question of when there exists a f.g. $\Lambda$-module $M$ such that $X \cong \hat{M}$, the $J(R)$-adic completion of $M$. Our answers sharpen the package deal theorem of Section 3 in this less general setting (i.e. orders in semisimple artinian rings) and our proofs use the results of Section 3 . 
Let $\Gamma$ be a normalization of $\Lambda$. One difference between our situation and the familiar classical one is that, when $\Gamma$ is not module-finite over $R, \hat{R}$ has nilpotent elements and therefore $\hat{\Lambda}=\hat{R} \otimes_{R} \Lambda$ has nilpotent ideals.

A basic necessary condition for the existence of $M$ is that $X_{Q}$ be a projective $\Lambda_{Q}$-module. This holds because the $\operatorname{ring} \Lambda_{Q}$ is semisimple artinian, hence the $\Lambda_{Q}$-module $M_{Q}$ must be projective; and this implies that $\hat{M}_{Q}=\hat{R} \otimes_{R} M_{Q}$ is a projective module over $\hat{\Lambda}_{Q}=\hat{R} \otimes_{R} \Lambda_{Q}$. We give two answers to the question of when $M$ exists.

The first answer focuses on the ring $\ddot{\Gamma}=\hat{R} \otimes_{R} \Gamma$. The notation is chosen to emphasize that $\ddot{\Gamma}$ is not an "adic" completion of $\Gamma$ when $\Gamma$ is not module-finite over $R$. Nor is it noetherian. (We are grateful to W. Heinzer for showing us this latter fact in the commutative case.) However we show that $\ddot{\Gamma}$ is semiperfect: idempotents can be lifted modulo $J(\ddot{\Gamma})$, and $\ddot{\Gamma} / J(\ddot{\Gamma})$ is a semisimple artinian ring. Moreover, $\ddot{\Gamma}_{Q}=\hat{\Lambda}_{Q}$ since $\Gamma_{Q}=\Lambda_{Q}$; and $\hat{\Lambda}_{Q}$ is therefore the natural artinian quotient ring of both $\ddot{\Gamma}$ and $\hat{\Lambda}$ when $\Lambda$ has no artinian ring direct summands.

Let the indecomposable ring direct summands of $\Gamma$ be $\Gamma_{k}$ (where $k$ runs through some finite index set). Thus $\Gamma=\bigoplus_{k} \Gamma_{k}$. As already mentioned in the discussion of normalizations, each $\Gamma_{k}$ is a classical maximal order over a Dedekind domain in a simple artinian ring. Since $R$ is semilocal, $\Gamma_{k}$ is a full matrix ring over a (possibly noncommutative) principal ideal domain. Therefore $\Gamma_{k}$ has, up to isomorphism, only one indecomposable f.g. projective left module. This module is isomorphic to $\Gamma_{k} e_{k}$, where $e_{k}$ is a primitive idempotent of $\Gamma_{k}$. The image $\bar{e}_{k}$ of $e_{k}$ in $\bar{\Gamma}_{k}=$ $\Gamma_{k} / J\left(\Gamma_{k}\right)$ is again idempotent, but not necessarily primitive. Since the ring $\bar{\Gamma}_{k}$ is semisimple artinian, we have a decomposition $\bar{\Gamma}_{k} \bar{e}_{k} \cong \bigoplus_{i}\left(S_{k i}\right)^{s_{k i}}$ where each $S_{k i}$ is a simple $\bar{\Gamma}_{k}$-module generated by a primitive idempotent of $\bar{\Gamma}_{k}$. We call these multiplicities $s_{k i}$ the splitting numbers of $\Gamma$.

Let $\ddot{\Gamma}_{k}=\hat{R} \otimes_{R} \Gamma_{k}$. It turns out that $\ddot{\Gamma}_{k} / J\left(\ddot{\Gamma}_{k}\right)=\bar{\Gamma}_{k}$. Since the ring $\ddot{\Gamma}_{k}$ is semiperfect, and hence idempotents lift from $\bar{\Gamma}_{k}$ to $\ddot{\Gamma}_{k}$, each $S_{k i}$ is the image modulo $J\left(\ddot{\Gamma}_{k}\right)$ of a unique indecomposable f.g. projective $\ddot{\Gamma}_{k}$-module $\ddot{U}_{k i}$ and every indecomposable f.g. projective $\ddot{\Gamma}$-module is isomorphic to some $\ddot{U}_{k i}$. Moreover, the $Q(R)$-localizations $\left(\ddot{U}_{k i}\right)_{Q}$ form a set of representatives of the isomorphism classes of indecomposable f.g. projective $\hat{\Lambda}_{Q}=\ddot{\Gamma}_{Q}$-modules. (The proof of this last fact takes more work than one might expect.) In particular, assuming our necessary condition that $X_{Q}$ is $\Lambda_{Q}$-projective, there is a decomposition $X_{Q} \cong \bigoplus_{k i}\left[\left(\ddot{U}_{k i}\right)_{Q}\right]^{\rho_{k i}}$. We call $\left(\left(\rho_{k i}\right)\right)$ the array of ranks of $X$.

Our package deal theorem for completions of orders then states that $M$ exists if and only if each row $\left(\rho_{k 1}, \rho_{k 2}, \ldots\right)$ of the array of ranks of $X$ is an integer multiple of the corresponding row $\left(s_{k 1}, s_{k 2}, \ldots\right)$ of the array of splitting numbers of $\Gamma$. Conceptually, this states that $X_{Q} \cong \hat{R} \otimes_{R} P_{Q}$ for some f.g. projective $\Gamma$-module $P$, exactly as in the case of classical orders!

Our other answer to the question about the existence of $M$ avoids mention of the non-noetherian ring $\ddot{\Gamma}$, replacing it by $\hat{\Omega}=\hat{R} \otimes \Omega$, the $J(R)$-adic completion of any convenient splitting $R$-order $\Omega \subseteq \Gamma$. Since completions of orders are semiperfect, each simple $\bar{\Gamma}_{k}$-module $S_{k i}$ lifts to a unique indecomposable f.g. projective $\hat{\Omega}$-module $U_{k i}$, and the localizations $\left(U_{k i}\right)_{Q}$ again paramaterize the isomorphism classes of indecomposable f.g. projective $\hat{\Lambda}_{Q}$-modules. Thus any f.g. $\hat{\Lambda}$-module $X$ such that $X_{Q}$ is $\Lambda_{Q}$-projective yields the same array of ranks as before, via the 
decomposition $X_{Q} \cong \bigoplus_{k i}\left[\left(U_{k i}\right)_{Q}\right]^{\rho_{k i}}$, and the package deal theorem in the previous paragraph again applies. As before, this is equivalent to saying that $M$ exists if and only if $X_{Q} \cong \hat{P}_{Q}$ (the $Q$-localization of the completion of $P$ ) for some f.g. projective $\Omega$-module.

The approach to the package deal theorem, via splitting orders, is needed in the proof of the version that involves $\ddot{\Gamma}$, and the splitting-order version is applied extensively in our analysis of the Krull-Schmidt problem in [LO].

An interesting fact about the mysterious ring $\ddot{\Gamma}$ deserves to be mentioned here: Modulo its nilradical it becomes noetherian and also a maximal order in its semisimple artinian quotient ring.

Artinian pairs. When looking only at f.g. torsionfree $\Lambda$-modules $M$ there is a much easier tool than completions that one can use to study direct-sum relations of $\Lambda$-modules. One can associate with $M$ a "module" $\Phi(M)$ over the pair of artinian rings $\Lambda / C$ and $\Omega / C$, where $\Omega$ is a splitting order such that $\Omega M$ is $\Omega$-projective, and $C$ is a conductor ideal for $\Lambda$ and $\Omega$. $[\Phi(M)$ consists of a pair of actual modules, one over each of the rings $\Lambda / C$ and $\Omega / C$.] As with completions, the two critical properties hold: (i) The Krull-Schmidt theorem holds for f.g. modules over artinian pairs; and (ii) $\Phi(M) \cong \Phi(N)$ if and only if $M \cong N$. Ultimately, one looks at the same array of ranks, whether using completions or artinian pairs, as we show at the end of Section 6.

We use artinian pairs whenever possible in [LO]. But when dealing with nontorsionfree modules, there does not seem to be any alternative to the more powerful (but more difficult to use) technique of completions.

Remark 1.2 (Artinian ring direct summands). Much of this paper does not work smoothly if the ring $\Lambda$ [equivalently, its center $Z(\Lambda)$ ] has artinian ring direct summands.

For example, let $\Lambda=\mathbf{Z} \oplus \mathbf{Z} / 5 \mathbf{Z}$ with $\mathbf{Z}$ the ring of integers. If $\Lambda$ is considered as an algebra over $R=\mathbf{Z}$ then $\Lambda_{Q}=\mathbf{Q}$, the rational numbers. However, if $\Lambda$ is considered as an algebra over $R=\Lambda$, then $\Lambda_{Q}=\mathbf{Q} \oplus \mathbf{Z} / 5 \mathbf{Z}$.

Moreover, this paper has nothing new to say about artinian rings. The way we deal with this is to note that since $R$ [usually $=Z(\Lambda)$ ] is noetherian it has a decomposition

$$
R=R^{\prime} \oplus A
$$

in which $A$ is artinian and $R^{\prime}$ has no artinian direct summands $\neq 0$. If $M$ is any $R$-module, this decomposition of $R$ induces a decomposition $M=M^{\prime} \oplus B$ where $M^{\prime}$ and $B$ are modules over $R^{\prime}$ and $A$ respectively. Whatever we are proving is usually trivially true or trivially false for $B$. We therefore assume that $R=R^{\prime}$ for the rest of the proof, adding the hypothesis "if $R$ has no artinian ring direct summands" when appropriate.

For semiprime rings (rings without nilpotent ideals) this dichotomy works in the noncommutative as well as in the commutative case. The following is proved in [GL '89, (1.4.3)]:

(1.2.1) Every semiprime module-finite $R$-algebra $\Lambda^{\prime}$ is a direct sum of two $R$-algebras $\Lambda^{\prime}=\Lambda \oplus A$, where $A$ is an artinian ring, $\Lambda$ is an $R \cdot 1_{\Lambda}$-order in a semisimple artinian ring, and $\Lambda$ has no nonzero $R$-submodules of finite length and no nonzero $\Lambda$-submodules of finite length. 
The (known) facts about commutative rings that make the situation $R=R^{\prime}$ work more smoothly are listed in the following lemma, in the form in which we shall need them. $J(R)$ denotes the Jacobson radical of $R$.

Lemma 1.3. Suppose that $R$ has no artinian ring direct summands $\neq 0$. Then:

(i) No minimal prime ideal of $R$ is maximal; and

(ii) $\mathbf{m} \neq \mathbf{m}^{2}$ for every maximal ideal $\mathbf{m}$ of $R$.

(iii) Suppose that $R$ is semilocal. Then $J(R)_{Q}=R_{Q}$; that is, $J(R)-\bigcup Q$ is nonempty. [Recall that if $R$ has no nilpotent elements, $R-\bigcup Q$ is the set of regular elements (non-zero-divisors) of $R$.]

Proof. Since idempotents can be lifted modulo nil ideals, it suffices to prove (i) and (ii) for $R / N$, where $N$ is the nilradical of $R$. So we can suppose that $R$ has no nilpotent elements.

(i). Since $R$ has no nonzero nilpotent elements, the intersection of the minimal primes is zero. If some minimal prime $\mathbf{p}$ were also maximal, we would therefore have $R=I \oplus \mathbf{p}$ where $I$ is the intersection of the minimal primes $\neq \mathbf{p}$. But then $I$ would be a minimal ideal of $R$, a contradiction since $R$ has no artinian ring direct summands.

(ii). Let $\mathbf{m}$ be a maximal ideal of $R$ such that $\mathbf{m}=\mathbf{m}^{2}$. Since $R$ is noetherian, Krull's intersection theorem yields $\mathbf{m}=R d$ for some idempotent element $d$. Thus $\mathbf{m}$ is a direct summand of $R$. The complementary summand is a minimal ideal, a contradiction since $R$ has no artinian direct summands.

(iii). If (iii) were false then $J(R)$ would be contained in the union of the minimal primes of $R$, hence in some minimal prime $\mathbf{p}$. Therefore some maximal ideal $\mathbf{m}$ would be contained in $\mathbf{p}$; hence $\mathbf{m}$ would be a minimal prime, contrary to (i).

The following lemma describes the type of ideal we use for a conductor ideal in both this paper and [LO]. Recall that " $R$-order" was defined in (1.1.1).

Lemma 1.4. Let $R$ be semilocal and let $\Lambda$ and $\Omega$ be $R$-orders in the semisimple artinian ring $\Lambda_{Q}$ such that $\Lambda \subseteq \Omega \subseteq \Lambda_{Q}$. Assume that $Z(\Lambda)$ has no artinian ring direct summands. Then $\Lambda$ and $\Omega$ have a common ideal $C$ such that

(i) $\Lambda / C$ and $\Omega / C$ are $R$-modules of finite length (hence artinian rings);

(ii) $C \subseteq J(\Lambda)$ [equivalently, $C \subseteq J(\Omega)$ ], where $J(\ldots)$ denotes the Jacobson radical; and

(iii) $C_{Q}=\Lambda_{Q}\left(=\Omega_{Q}\right)$ (so $C$ contains at least one central unit of $\Lambda_{Q}$ ).

We call any common ideal $C$ of $\Lambda$ and $\Omega$ satisfying (i) a conductor ideal for $\Lambda$ and $\Omega$. We usually choose $C$ to take advantage of properties (ii) and (iii). Incidentally, (ii) and (iii) become false if $Z(\Lambda)$ is allowed to have artinian ring direct summands, as is easily seen.

Proof. We may assume that $R=Z(\Lambda)$. In particular, $R$ has no nilpotent elements since any such element would generate a nilpotent ideal of $\Lambda$. By Lemma 1.3 there is an element $c \in J(R)-\bigcup Q$. Since $R$ has no nilpotent elements, $c$ is a regular element in $R$.

Since $\Lambda_{Q}=\Omega_{Q}$ and $\Omega$ is a finitely generated $R$-module, there is an element $d \in R-\bigcup Q$ such that $\Omega d \subseteq \Lambda$. Let $C=\Omega c d$. Then $C$ is a common ideal of $\Lambda$ and $\Omega$, and (iii) holds since $C$ contains the unit $c d$ of $R_{Q}$, hence of $\Lambda_{Q}$. Since $R$ has Krull dimension 1 and $c d$ is outside of every minimal prime of $R$, the ring $R / R c d$ is artinian. So (i) holds, by module-finiteness of $\Lambda$ and $\Omega$ over $R$. 
Statement (ii) holds because $J(R) \Lambda \subseteq J(\Lambda)$. The parenthetical statement in (ii) holds because of the quasi-regularity property of the radical.

Lemma 1.5. If $\Lambda$ [equivalently $Z(\Lambda)$ ] has no artinian ring direct summands then $\Lambda_{Q(R)}=\Lambda_{Q(Z(\Lambda))}$ [i.e. both localizations invert the same set of elements of $\left.Z(\Lambda)\right]$.

Proof. Let $S=Z(\Lambda)$. It suffices to prove that $S_{Q(R)}=S_{Q(S)}$. In order to state this more precisely, let $\nu: R \rightarrow S$ denote the natural map $\nu(r)=r \cdot 1_{S}$. We want to show that inverting the elements of the subset $\nu[R-Q(R)]$ of $S$ yields the same localization as inverting the elements of $S-Q(S)$; that is, both localizations ultimately invert the same elements of $S$. Since the proof is more subtle than one might expect, and the truth of the lemma depends upon on the fact that $R$ has Krull dimension 1, we begin by reviewing some basic facts.

Let $H$ be a multiplicatively closed subset of $S$, and $P$ a prime ideal of $S$. We say " $P$ survives in $S_{H}$ " if $P_{H} \neq S_{H}$. This happens if and only if $P \cap H=\varnothing$; and when $P$ survives, $P_{H}$ is a prime ideal of $S_{H}$. The precise set of elements of $S$ that become units in $S_{H}$ is the complement of the union of the set of prime ideals of $S$ that survive in $S_{H}$. Therefore if $K$ is another multiplicatively closed subset of $S$, we have $S_{H}=S_{K}$ if and only if the same prime ideals of $S$ survive in both localizations.

Since $S$ has no artinian direct summands, no maximal ideal of $S$ is a minimal prime ideal [Lemma 1.3].

We now prove that a prime ideal of $S$ survives in $S_{Q(R)}$ if and only if it is a minimal prime ideal. Consider first a minimal prime ideal $\mathbf{p}$ of $S$. Since $\mathbf{p}$ is not a maximal ideal we have a proper inclusion $\mathbf{p} \subset \mathbf{m}$ for some maximal ideal m of $S$. Since $S$ is module-finite over $R$, it is integral over $\nu(R)$. Therefore, by the incomparability theorem for prime ideals we have $\mathbf{p} \cap \nu(R) \subset \mathbf{m} \cap \nu(R)$. Let $\mathbf{p}^{\prime}=\nu^{-1}[\mathbf{p} \cap \nu(R)]$ and $\mathbf{m}^{\prime}=\nu^{-1}[\mathbf{m} \cap \nu(R)]$. Then $\mathbf{p}^{\prime} \subset \mathbf{m}^{\prime}$. Since $R$ has Krull dimension 1 the prime ideal $\mathbf{p}^{\prime}$ is therefore a minimal prime. Now let $x$ be any element of $R-Q(R)$. If $\nu(x) \in \mathbf{p}$ then $x \in \mathbf{p}^{\prime}$, which is impossible since $\mathbf{p}^{\prime} \in Q(R)$. Therefore $\mathbf{p}$ survives in $S_{Q(R)}$.

Now let $\mathbf{m}$ be a maximal ideal of $S$. We show that $\mathbf{m}$ does not survive in $S_{Q(R)}$. By choosing a minimal prime ideal $\mathbf{p} \subset \mathbf{m}$ we show, as before, that $\mathbf{m}^{\prime}=$ $\nu^{-1}[\mathbf{m} \cap \nu(R)]$ is a maximal ideal of $R$ and $\mathbf{m}^{\prime}$ is not a minimal prime ideal. The maximal ideal $\mathbf{m}^{\prime}$ cannot be contained the union of the minimal prime ideals of $R$, because $\mathbf{m}^{\prime}$ would then be contained in some minimal prime, and hence be minimal. Therefore there exists an element $x \in \mathbf{m}^{\prime}-\bigcup Q(R)$. But then $\nu(x) \in \mathbf{m}$ shows that m does not survive in $S_{Q(R)}$, as desired.

Finally, the case $R=S$ yields the well-known fact that the prime ideals of $S$ that survive in $S_{Q(S)}$ are precisely the minimal primes, completing the proof of our lemma.

Lemma 1.6. Let $\Lambda$ be any module-finite $R$-algebra, as in Notation 1.1. Then

(i) The correspondence $S \mapsto M=\operatorname{ann}_{\Lambda} S$ (the annihilator of $S$ in $\Lambda$ ) is a bijection between the set of isomorphism classes of simple (left) $\Lambda$-modules $S$ and the set of maximal (2-sided) ideals $M$ of $\Lambda$. In particular, $J(\Lambda)$ equals the intersection of the set of maximal ideals of $\Lambda$.

(ii) For every maximal ideal $M$ of $\Lambda$, the simple ring $\Lambda / M$ is artinian.

(iii) Every $\Lambda$-module of finite length has finite length as an $R$-module. 
(iv) Suppose that $\Lambda$ has no artinian ring direct summands. Then a f.g. $\Lambda$-module $U$ has finite length if and only if $U_{Q}=0$.

Proof. (i) and (ii). It suffices to show that an ideal $M$ of $\Lambda$ is primitive (i.e. the annihilator of a simple left module) if and only it is maximal. For the nontrivial implication suppose that $M$ is primitive. Then $\Lambda / M$ is a primitive ring that is module finite over a commutative ring, and it therefore satisfies a polynomial identity [MR '87, (13.1.13)]. By Kaplansky's theorem [MR '87, (13.3.8)] $\Lambda / M$ is therefore a simple artinian ring.

(iii). It suffices to show that every simple $\Lambda$-module $S$ has finite length as an $R$-module. Let $M=\operatorname{ann}_{\Lambda} S$. Then $\Lambda / M$ is a simple artinian ring by statements (i) and (ii), and its simple module $S$ is therefore isomorphic to a submodule of $\Lambda / M$. Since $\Lambda / M$ is module-finite over $R$, so therefore is $S$.

(iv). We may suppose that $R=Z(\Lambda)$, by Lemma 1.5. Then $R$ has no artinian ring direct summands. We claim that $S_{Q}=0$ for every simple $R$-module $S$.

We have $S=R / \mathbf{m}$ for some maximal ideal $\mathbf{m}$; and $\mathbf{m}$ is not a minimal prime ideal by Lemma 1.3. Hence $\mathbf{m}$ is not contained in the union of the minimal prime ideals of $R$; and it follows that $\mathbf{m}_{Q}=R_{Q}$, and therefore $(R / \mathbf{m})_{Q}=0$, as claimed.

Now suppose that the $\Lambda$-module $U$ has finite length. Then it has finite length as an $R$-module, by statement (iii). To show that $U_{Q}=0$ it suffices to show that $S_{Q}=0$ for every composition factor $S$ of the $R$-module $U$. This was done in the previous claim.

Conversely, suppose $U_{Q}=0$. Then $d U=0$ for some $d \in R-\bigcup Q$. Therefore $U$ is a f.g. module over the ring $R / R d$, which is artinian by our dimension 1 hypothesis. Consequently, $U$ has finite length as an $R$-module, and hence as a $\Lambda$-module. (This half of the proof did not require the hypothesis about no artinian direct summands.)

\section{PaCkages of Localizations}

Consider a family $\{X(\mathbf{m}) \mid \mathbf{m} \in \operatorname{maxspec}(R)\}$, with each $X(\mathbf{m})$ a f.g. $\Lambda_{\mathbf{m}^{-}}$ module. In this section we consider the question of whether there is a f.g. $\Lambda$-module $M$ such that $M_{\mathbf{m}} \cong X(\mathbf{m})$ for all $\mathbf{m}$, tht is, whether $\{X(\mathbf{m})\}$ is the "package" of all localizations of some f.g. $\Lambda$-module at the maximal ideals $\mathbf{m}$ of $R$. Our proof forces us to answer a related question for submodules of a given module $M$ : Given a $\Lambda_{\mathbf{m}}$-submodule $X(\mathbf{m}) \subseteq M_{\mathbf{m}}$ for each $\mathbf{m}$, when is there a submodule $X \subseteq M$ such that every $X(\mathbf{m})$ equals $M_{\mathbf{m}}$ ? In both situations the spirit of the answer is that, subject to some obvious restrictions, one can specify any finite set of localizations. Moreover, if this finite set is chosen large enough, then the structure of the remaining $\mathbf{m}$-localizations is determined by their $Q$-localization.

As an application we show that indecomposability of any f.g. $\Lambda$-module is semilocally determined.

Notation 2.1. The letter $\mathbf{m}$ always denotes a maximal ideal of $R$, and (a $\forall \mathbf{m}$ ) means "for almost all maximal ideals $\mathbf{m}$ of $R$ ", that is, for all but finitely many m. We say that a set $\mathcal{M}$ of maximal ideals of $R$ covers $\operatorname{minspec}(R)$ if for every minimal prime ideal $\mathbf{p}$ of $R$ there is a maximal ideal $\mathbf{m} \in \mathcal{M}$ such that $\mathbf{m} \supseteq \mathbf{p}$.

The following lemma will be used frequently, sometimes without explicit acknowledgement. 
Lemma 2.2. (i) $R_{Q} \cong \bigoplus_{\mathbf{p} \in \operatorname{minspec}(R)} R_{\mathbf{p}}$. Therefore $M_{Q} \cong \bigoplus_{\mathbf{p} \in \operatorname{minspec}(R)} M_{\mathbf{p}}$ for every $\Lambda$-module $M$.

(ii) $R_{\mathbf{m}} \otimes_{R} R_{\mathbf{p}}=0$ whenever $\mathbf{m} \in \operatorname{maxspec}(R), \mathbf{p} \in \operatorname{minspec}(R)$, and $\mathbf{m} \nsupseteq \mathbf{p}$.

Proof. (i) holds since every commutative artinian ring is the direct sum of its localizations at its finite number of prime (necessarily maximal) ideals.

(ii) Take any $x \in \mathbf{p}-\mathbf{m}$. Then $x$ becomes a unit in $R_{\mathbf{m}}$ and becomes nilpotent in the artinian ring $R_{\mathbf{p}}$. But $x^{n} / 1=0$ implies that $1 \otimes 1=\left(1 / x^{n}\right) \otimes\left(x^{n} / 1\right)=0$.

Lemma 2.3. (i) $R-\bigcup Q$ localizes. In other words, $\left(R_{Q}\right)_{\mathbf{m}}=\left(R_{\mathbf{m}}\right)_{Q\left(R_{\mathbf{m}}\right)}$.

(ii) Let $X$ be an $R_{\mathbf{m}}$-module, for some $\mathbf{m}$. Then $X$ is $Q(R)$-torsionfree if and only $X$ is $Q\left(R_{\mathbf{m}}\right)$-torsionfree. (Hence $X$ can unambiguously be called $Q$-torsionfree.)

Proof. (i) We have $R_{Q} \cong \bigoplus_{\mathbf{p} \in \operatorname{minspec}(R)} R_{\mathbf{p}}$ by Lemma 2.2. Tensoring with $R_{\mathbf{m}}$ and using Lemma 2.2(ii) yields $\left(R_{Q}\right)_{\mathbf{m}}=\bigoplus_{\mathbf{p} \subseteq \mathbf{m}} R_{\mathbf{p}} \otimes_{R} R_{\mathbf{m}}$. Then note that $R_{\mathbf{p}} \otimes_{R} R_{\mathbf{m}}=R_{\mathbf{p}}$ when $\mathbf{p} \subseteq \mathbf{m}$.

(ii) This is an immediate consequence of (i).

The fact that $R_{Q}$ is an artinian ring imposes the following basic finiteness restrictions on localizations.

Lemma 2.4. Let $L, M, N$ be f.g. $\Lambda$-modules.

(i) If $N \subseteq M$ and $M_{Q}=N_{Q}$, then $M_{\mathbf{m}}=N_{\mathbf{m}}(\mathrm{a} \forall \mathbf{m})$.

(ii) If $L_{Q} \cong M_{Q}$, then $L_{\mathbf{m}} \cong M_{\mathbf{m}}(\mathrm{a} \forall \mathbf{m})$.

(iii) If $L_{Q} \mid M_{Q}$ (i.e. $L_{Q}$ is isomorphic to a direct summand of $M_{Q}$ ) then $L_{\mathbf{m}} \mid M_{\mathbf{m}}$ $(\mathrm{a} \forall \mathbf{m})$.

(iv) $M_{\mathbf{m}}$ is $Q$-torsionfree [equivalently, $Q\left(R_{\mathbf{m}}\right)$-torsionfree] ( $\left.\mathbf{a} \forall \mathbf{m}\right)$.

(v) There is a finite set $\mathcal{M}$ of maximal ideals of $R$ such that the natural map $M \rightarrow M_{\mathcal{M}}$ is one-to one, and remains one-to-one if $\mathcal{M}$ is replaced by any larger finite set of maximal ideals.

Proof. (i) Since $M$ is a finitely generated $\Lambda$-module and $M_{Q}=N_{Q}$ we have $d M \subseteq N$ for some $d \in R-\bigcup Q$. Since $d$ is outside of all minimal primes of our ring $R$ of dimension 1 , the ring $R / R d$ is artinian, and so only finitely many maximal ideals of $R$ contain $d$. For any other maximal ideal $\mathbf{m}, d / 1$ is a unit in $R_{\mathbf{m}}$, hence $M_{\mathbf{m}}=N_{\mathbf{m}}$.

(ii) and (iii) See [GL '89, 2.1(i) and (iii)].

(iv) Let $K=\operatorname{ker}\left(M \rightarrow M_{Q}\right)$. Then $K_{Q}=\operatorname{ker}\left(M_{Q} \rightarrow M_{Q}\right)=0$. Therefore $K_{\mathbf{m}}=0(\mathrm{a} \forall \mathbf{m})$, by (i). But $K_{\mathbf{m}}=\operatorname{ker}\left(M_{\mathbf{m}} \rightarrow\left(M_{\mathbf{m}}\right)_{Q}\right)$, and $\left(M_{\mathbf{m}}\right)_{Q}$ can be identified with $\left(M_{\mathbf{m}}\right)_{Q\left(R_{\mathbf{m}}\right)}$ by Lemma 2.3 .

(v) Let $\mathcal{M}$ be the finite set of maximal ideals such that $M_{\mathbf{m}}$ is not $Q$-torsionfree, and enlarge $\mathcal{M}$, if neccessary, so that it covers $\operatorname{minspec}(R)$. To see that the natural map $M \rightarrow M_{\mathcal{M}}$ is one-to-one, take $m \in M$ such that $(m / 1)_{\mathbf{m}}=0$ for all $\mathbf{m} \in \mathcal{M}$. It suffices to show that $(m / 1)_{\mathbf{m}}=0$ for all $\mathbf{m} \notin \mathcal{M}$. Since $\mathcal{M}$ covers $\operatorname{minspec}(R)$ we have $(m / 1)_{Q}=0$. Therefore $(m / 1)_{\mathbf{m}}=0$ whenever $M_{\mathbf{m}}$ is $Q$-torsionfree. This includes all $\mathbf{m} \notin \mathcal{M}$.

Now consider a f.g. $\Lambda$-module $M$. We wish to change a single localization $M_{\mathbf{n}}$ to some specified $R_{\mathbf{n}}$-submodule of it, without changing any other $M_{\mathbf{m}}$. What restrictions apply? The possibly surprising answer is that there are no restrictions other than the obvious one: do not change $\left(M_{\mathbf{n}}\right)_{Q}$, as we show in the next lemma. 
Lemma 2.5. Let $M$ be a f.g. $\Lambda$-module, $\mathbf{n} \in \operatorname{maxspec}(R)$, and $X$ a $\Lambda_{\mathbf{n}}$-submodule of $M_{\mathbf{n}}$ such that $X_{Q}=\left(M_{\mathbf{n}}\right)_{Q}$. Then there is a $\Lambda$-submodule $N \subseteq M$ such that $N_{\mathbf{n}}=X$ and $N_{\mathbf{m}}=M_{\mathbf{m}}$ for all other maximal ideals $\mathbf{m}$.

Proof. We may assume that $X \neq M_{\mathbf{n}}$. We find $N$ by means of two approximations. First we find a submodule $L \subseteq M$ such that

$$
L_{\mathbf{n}}=X \quad \text { and } \quad L_{Q}=M_{Q}
$$

Then, since $L_{Q}=M_{Q}$ we have $L_{\mathbf{m}}=M_{\mathbf{m}}(\mathrm{a} \forall \mathbf{m})$, by Lemma 2.4. Let $\mathcal{F}$ be the finite set of maximal ideals $\mathbf{m} \neq \mathbf{n}$ such that $L_{\mathbf{m}} \neq M_{\mathbf{m}}$. We find an element $e \in R$ such that

$$
e M_{\mathbf{n}} \subseteq L_{\mathbf{n}}(=X) \quad \text { and } \quad e M_{\mathbf{m}}=M_{\mathbf{m}} \quad(\forall \mathbf{m} \in \mathcal{F}) .
$$

Then $N=L+e M$ is the desired submodule of $M$.

Since $X_{Q}=\left(M_{\mathbf{n}}\right)_{Q}$, finite generation of $M_{\mathbf{n}}$ yields $b \in R-\bigcup Q$ such that $b M_{\mathbf{n}} \subseteq X$. Since $X \neq M_{\mathbf{n}},(b / 1)_{\mathbf{n}}$ is a nonunit in the local ring $R_{\mathbf{n}}$, that is, $(b / 1)_{\mathbf{n}} \in \mathbf{n}_{\mathbf{n}}$. Nilpotence of the radical of the artinian ring $R_{\mathbf{n}} / R_{\mathbf{n}} b$ then yields a positive integer $s$ such that $\mathbf{n}_{\mathbf{n}}^{s} \subseteq R_{\mathbf{n}} b$. Thus

$$
b M_{\mathbf{n}} \subseteq X \quad \text { and } \quad \mathbf{n}_{\mathbf{n}}^{s} \subseteq R_{\mathbf{n}} b, \quad \text { hence } \quad \mathbf{n}^{s} M_{\mathbf{n}} \subseteq X .
$$

Let $I=\bigcap\{\mathbf{p} \in \operatorname{minspec}(R) \mid \mathbf{p} \nsubseteq \mathbb{n}\}$. Then $\mathbf{n}^{s}+I=R$, so the Chinese Remainder Theorem yields $d \in R$ such that

$$
d \equiv b \quad\left(\bmod \mathbf{n}^{s}\right) \quad \text { and } \quad d \equiv 1 \quad(\bmod I) .
$$

Let $L^{\prime}$ be the $\Lambda$-submodule of $M$ generated by the numerators of some finite set of $\Lambda_{\mathbf{n}}$-generators of $X$. We claim that $L=L^{\prime}+d M$ is as required in (2.5.1).

To see that $L_{\mathbf{n}}=X$ it suffices to note that $d M_{\mathbf{n}} \subseteq X$. To see that $L_{Q}=M_{Q}$ it suffices to check that $L_{\mathbf{p}}=M_{\mathbf{p}}(\forall \mathbf{p} \in \operatorname{minspec}(R))$. If $\mathbf{p} \subseteq \mathbf{n}$ we have

$$
L_{\mathbf{p}}=\left(L_{\mathbf{n}}\right)_{\mathbf{p}}=X_{\mathbf{p}}=\left(X_{Q}\right)_{\mathbf{p}}=\left(\left(M_{\mathbf{n}}\right)_{Q}\right)_{\mathbf{p}}=M_{\mathbf{p}}
$$

Suppose therefore that $\mathbf{p} \nsubseteq \mathbf{n}$. Then $d$ becomes a unit in $R_{\mathbf{p}}$ and so we again have $L_{\mathbf{p}}=M_{\mathbf{p}}$. This completes the proof of (2.5.1).

Now we find $e$ such that (2.5.2) holds. Let $b$ again be as in (2.5.3). Then the Chinese Remainder Theorem yields $e \in R$ such that $e \equiv b\left(\bmod \mathbf{n}^{s}\right)$ and $e \equiv 1$ $(\bmod \mathbf{m})(\forall \mathbf{m} \in \mathcal{F})$, completing the proof.

Lemma 2.4 together with repeated use of Lemma 2.5 immediately yields:

Package Deal Theorem 2.6 (Localizations of Submodules). Let $M$ be a f.g. $\Lambda$ module, and for each $\mathbf{m} \in \operatorname{maxspec}(R)$ let $X(\mathbf{m})$ be a f.g. $\Lambda_{\mathbf{m}}$-submodule of $M_{\mathbf{m}}$ such that $X(\mathbf{m})_{Q}=\left(M_{\mathbf{m}}\right)_{Q}$. Then there is a $\Lambda$-submodule $N \subseteq M$ such that $N_{\mathbf{m}}=X(\mathbf{m})(\forall \mathbf{m})$ if and only if $(\mathrm{a} \forall \mathbf{m}) X(\mathbf{m})=M_{\mathbf{m}}$.

The previous theorem can be sharpened if $M$ is $Q$-torsionfree. In that situation every $M_{\mathbf{m}} \subseteq M_{Q}$, so one can try to replace some of the submodules $M_{\mathbf{m}}$ by f.g. $\Lambda_{\mathbf{m}}$-submodules $X(\mathbf{m}) \subseteq M_{Q}$ that are not contained in $M_{\mathbf{m}}$. The following result does this, extending [GL '88, 2.6] which was proved under the assumption that $\Lambda$ is an order in a semisimple artinian ring.

Corollary 2.7. Let $M$ be a f.g. Q-torsionfree $\Lambda$-module, and for each maximal ideal $\mathbf{m} \in \operatorname{maxspec}(R)$ let $X(\mathbf{m})$ be a f.g. $\Lambda_{\mathbf{m}}$-submodule of $M_{Q}$ such that $X(\mathbf{m})_{Q}=$ $\left(M_{\mathbf{m}}\right)_{Q}$. Then there is a f.g. $\Lambda$-submodule $N \subseteq M_{Q}$ such that $N_{\mathbf{m}}=X(\mathbf{m})(\forall \mathbf{m})$, if and only if $(\mathrm{a} \forall \mathbf{m}) X(\mathbf{m})=M_{\mathbf{m}}$. 
Proof. If. There exists $d \in R-\bigcup Q$ such that $(\forall \mathbf{m}) d X(\mathbf{m}) \subseteq M_{\mathbf{m}}$ because of our torsionfree and finite generation hypotheses, and the hypothesis that $X(\mathbf{m})=M_{\mathbf{m}}$ $(\mathrm{a} \forall \mathbf{m})$. Since $M$ is $Q$-torsionfree, $d^{-1} M$ is a well-defined $\Lambda$-submodule of $M_{Q}$. Therefore we can apply the previous theorem to the $\Lambda$-module $M^{\prime}=d^{-1} M$ and the family of $\Lambda_{\mathbf{m}}$-submodules $X(\mathbf{m})$ of $M_{\mathbf{m}}^{\prime}$. Since $d / 1$ is a unit in almost all localizations $R_{\mathbf{m}}$, the hypothesis $X(\mathbf{m})=M_{\mathbf{m}}(\mathrm{a} \forall \mathbf{m})$ shows that $X(\mathbf{m})=\left(M^{\prime}\right)_{\mathbf{m}}$ $(a \forall \mathbf{m})$; and then the previous theorem completes the proof of this half of the corollary.

Only if. Since $\left(M_{\mathbf{m}}\right)_{Q}=\left(N_{\mathbf{m}}\right)_{Q}(\forall \mathbf{m})$ we have $M_{Q}=N_{Q}$. As in the "if" part of the proof we find elements $c, d \in R-\bigcup Q$ such that $c M \subseteq N$ and $d N \subseteq M$. Since $c$ and $d$ become units in almost all localizations $R_{\mathbf{m}}$, we have $M_{\mathbf{m}}=N_{\mathbf{m}}(\mathrm{a} \forall \mathbf{m})$.

Remark 2.8. Let $M$ be a f.g. $\Lambda$-module and $(\forall \mathbf{m}) X(\mathbf{m})$ a $\Lambda_{\mathbf{m}}$-submodule of $M_{\mathbf{m}}$. But do not assume that $X(\mathbf{m})_{Q}=\left(M_{\mathbf{m}}\right)_{Q}$, which was assumed in Theorem 2.6. Is there a $\Lambda$-submodule $N \subseteq M$ such that $(\forall \mathbf{m}) N_{\mathbf{m}}=X(\mathbf{m})$ ? The answer is almost the same as in Theorem 2.6 but more difficult to state. We discuss it briefly here.

There is an obviously necessary $Q$-consistency condition, namely that $X(\mathbf{m})_{\mathbf{p}}=$ $X(\mathbf{n})_{\mathbf{p}}$ whenever $\mathbf{p} \in \operatorname{minspec}(R), \mathbf{m}, \mathbf{n} \in \operatorname{maxspec}(R)$ and $\mathbf{p} \subseteq \mathbf{m} \cap \mathbf{n}$. Assume that this condition is satisfied. For each $\mathbf{p} \in \operatorname{minspec}(R)$ choose a maximal ideal $\mathbf{m}(\mathbf{p}) \supseteq \mathbf{p}$ and define $X(Q)=\bigoplus_{\mathbf{p}} X(\mathbf{m}(\mathbf{p}))_{\mathbf{p}} \subseteq M_{Q}$. Because of $Q$-consistency, the $\Lambda_{Q}$-submodule $X(Q) \subseteq M_{Q}$ is independent of the particular choice of maximal ideals $\mathbf{m}(\mathbf{p})$.

Let $L=\left\{m \in M \mid(m / 1)_{Q} \in X(Q)\right\}$. Then $L_{Q}=X(Q)$, and we can now apply Theorem 2.6, with $L$ in place of $M$. Thus $N$ exists if and only if $X(\mathbf{m})=L_{\mathbf{m}}$ $(\mathrm{a} \forall \mathbf{m})$.

We now shift our attention to localizations of modules themselves, rather than localizations of submodules of a given module. Let $V$ be a f.g. $\Lambda_{Q}$-module. Then $V=L_{Q}$ for some f.g. $\Lambda$-module $L$. If $N$ is any other f.g. $\Lambda$-module such that $N_{Q} \cong L_{Q}$ then Lemma 2.4 shows that $N_{\mathbf{m}} \cong L_{\mathbf{m}}(\mathrm{a} \forall \mathbf{m})$. Our second package deal theorem shows that the hypothesis $N_{Q} \cong L_{Q}$ imposes no other restrictions on $N$.

Package Deal Theorem 2.9. (Localizations of Modules) Let L be a f.g. $\Lambda$-module, and for each $\mathbf{m}$ in some finite subset $\mathcal{M} \subseteq \operatorname{maxspec}(R)$, let $X(\mathbf{m})$ be a f.g. $\Lambda_{\mathbf{m}^{-}}$ module such that $X(\mathbf{m})_{Q} \cong\left(L_{\mathbf{m}}\right)_{Q}$. Then there is a f.g. $\Lambda$-module $N$ such that

(i) $N_{\mathbf{m}} \cong X(\mathbf{m})(\forall \mathbf{m} \in \mathcal{M})$, and

(ii) $N_{\mathbf{m}} \cong L_{\mathbf{m}}$ for all other maximal ideals $\mathbf{m}$.

Proof. We first note that the theorem is true when $R$ is semilocal. See, for example, [W '89, 1.11] or [HL '87, 1.6] for the commutative case $\Lambda=R$. These proofs work without change when $\Lambda$ is any module-finite, noncommutative $R$-algebra. (Ignore the torsionfreeness hypothesis in [HL '87].)

Now consider the situation that $R$ is not semilocal. We can enlarge $\mathcal{M}$ to any larger finite set of maximal ideals by defining $X(\mathbf{m})=L_{\mathbf{m}}$ for the new maximal ideals $\mathbf{m}$. So we can assume that $\mathcal{M}$ covers minspec $(R)$. By Lemma 2.4, we can further enlarge $\mathcal{M}$ so that $L_{\mathbf{m}}$ is $Q$-torsionfree for every $\mathbf{m} \notin \mathcal{M}$. By the semilocal case of the theorem there is a f.g. $\Lambda_{\mathcal{M}}$-module $Y$ such that $Y_{\mathbf{m}} \cong X(\mathbf{m})(\forall \mathbf{m} \in \mathcal{M})$.

We have $\left(Y_{\mathbf{m}}\right)_{Q} \cong X(\mathbf{m})_{Q}(\forall \mathbf{m} \in \mathcal{M})$, which, together with the hypothesis $X(\mathbf{m})_{Q} \cong\left(L_{\mathbf{m}}\right)_{Q}$, shows that $\left(Y_{\mathbf{m}}\right)_{Q} \cong\left(L_{\mathbf{m}}\right)_{Q}$. Since $\mathcal{M}$ covers minspec $(R)$ this 
yields, by Lemma 2.2 :

$$
L_{Q} \cong Y_{Q} .
$$

For a first approximation, let $N$ be the $\Lambda$-submodule of $Y$ generated by the numerators of some finite set of $\Lambda_{\mathcal{M}}$-generators of $Y$. Then $N_{\mathcal{M}}=Y$, and hence $N_{\mathbf{m}} \cong X(\mathbf{m})(\forall \mathbf{m} \in \mathcal{M})$. Since $\mathcal{M}$ covers $\operatorname{minspec}(R)$, we have $\left(L_{\mathcal{M}}\right)_{Q} \cong L_{Q}$ and $\left(N_{\mathcal{M}}\right)_{Q} \cong N_{Q}$. Therefore $N_{Q} \cong Y_{Q}$, which, together with (2.9.1), shows that $N_{Q} \cong L_{Q}$. Therefore $N_{\mathbf{m}} \cong L_{\mathbf{m}}(\mathrm{a} \forall \mathbf{m})$, by Lemma 2.4 .

Choose any one $\mathbf{n}$ of the finitely many maximal ideals such that this last isomorphism does not hold. It now suffices to show that $N_{\mathbf{n}}$ has a $\Lambda_{\mathbf{n}}$-submodule $X$ such that $X \cong L_{\mathbf{n}}$ and $X_{Q}=\left(N_{\mathbf{n}}\right)_{Q}$. For then Lemma 2.5 allows us to replace $N_{\mathbf{n}}$ by $X$ without changing any other localization of $N$.

Since $N_{Q} \cong L_{Q}$ we have $\left(N_{\mathbf{n}}\right)_{Q} \cong\left(L_{\mathbf{n}}\right)_{Q}$. Since $N_{\mathbf{n}}$ and $L_{\mathbf{n}}$ are both $Q$ torsionfree, we can therefore assume that they are both $\Lambda_{\mathbf{n}}$-submodules of $W=$ $\left(N_{\mathbf{n}}\right)_{Q}$. Finite generation of $L_{\mathbf{n}}$ over $\Lambda_{\mathbf{n}}$ shows that there exists $d \in R-\bigcup Q$ such that $d L_{\mathbf{n}} \subseteq N_{\mathbf{n}}$; and we may therefore take $X=d L_{\mathbf{n}}$.

The previous theorem avoids the question of the structure of the infinitely many localizations that we cannot change. Our next objective is to show that, when a sufficiently large finite number of localizations are discarded, the remaining ones "decompose like" their $Q$-localization.

Definitions 2.10. Let $X$ be a f.g. $\Lambda$-module. We say that $X$ decomposes like $X_{Q}$ if $X$ has a decomposition $X=\bigoplus_{i} Y_{i}$ where each $Y_{i}$ is indecomposable, each $\left(Y_{i}\right)_{Q}$ is indecomposable, and $Y_{i} \cong Y_{j}$ whenever $\left(Y_{i}\right)_{Q} \cong\left(Y_{j}\right)_{Q}$. Since many of our arguments involve counting the multiplicities of isomorphism classes of summands, it will be convenient to display these multiciplicites explicitly as follows.

(2.10.1) $\quad X \cong \bigoplus_{i=1}^{m}\left(X_{i}\right)^{e_{i}}$, where the $X_{i}$ are indecomposable pairwise nonisomorphic $\Lambda$-modules whose $Q$-localizations $\left(X_{i}\right)_{Q}$ are indecomposable and pairwise nonisomorphic.

We call the (isomorphism classes of the) $X_{i}$ the root summands of $X$. We will only be interested in these definitions when $R$ is semilocal, namely localizations of our original $R$.

Note 1. Such an $X$ can have indecomposable direct summands $U$ such that $U_{Q}$ is not indecomposable. Moreover, $X$ can have indecomposable direct summands $V$ such that $V_{Q}$ is again indecomposable, yet $V$ is not a root summand of $X$. See Examples 2.12. However, the root summands $X_{i}$ and their multiplicities $e_{i}$ are unique, as we show below.

Note 2. Our definition requires every $\left(X_{i}\right)_{Q}$ in $(2.10 .1)$ to be nonzero, since it is indecomposable. Therefore, if $X$ decomposes like $X_{Q}$, then $X$ is $Q$-torsionfree.

Lemma 2.11. Suppose that $R$ is semilocal and let $X$ be a f.g. $\Lambda$-module that decomposes like $X_{Q}$, with decomposition (2.10.1). Let $X \cong \bigoplus_{i=1}^{n}\left(Y_{i}\right)^{f_{i}}$ be another decomposition of this same form. Then $m=n$ and the $Y_{i}$ can be renumbered in such a way that every $X_{i} \cong Y_{i}$ and every $e_{i}=f_{i}$.

Proof. We shall refer to the decomposition $X \cong \bigoplus_{i}\left(Y_{i}\right)^{f_{i}}$ as decomposition $(2.10 .1)^{\prime}$. By taking the $Q$-localization of these two decompositions and applying the KrullSchmidt theorem for modules of finite length, we see that $m=n$ and, after a suitable renumbering of the $Y_{i}$, every $\left(X_{i}\right)_{Q} \cong\left(Y_{i}\right)_{Q}$ and $e_{i}=f_{i}$. Thus the nontrivial part of this proof is to show that every $X_{i} \cong Y_{i}$. 
Suppose $m=n=1$. In other words, suppose that $\left(X_{1}\right)^{e} \cong\left(Y_{1}\right)^{f}$. Then, as mentioned above, $e=f$. The desired isomorphism $X_{1} \cong Y_{1}$ is often expressed by saying that $e^{\text {th }}$ roots of $\Lambda$-modules are unique, when they exist, when $R$ is semilocal. This is easily proved in the present situation by taking the $J(R)$-adic completion and then applying the Krull-Schmidt theorem. To reduce to this situation, let $E$ be the endomorphism ring of $X$ (endomorphisms written on the right, for the rest of this proof). Then $E$ is a module-finite $R$-algebra. Moreover, the given decompositions (2.10.1) and (2.10.1)' of $X$ induce decompositions of the left $E$ module $E$, as displayed below,

$$
E \cong \bigoplus_{i}\left(E_{i}\right)^{e_{i}} \cong \bigoplus_{i}\left(F_{i}\right)^{e_{i}}
$$

where $E_{i}=\operatorname{Hom}_{\Lambda}\left(X, X_{i}\right)$ and $F_{i}=\operatorname{Hom}_{\Lambda}\left(X, Y_{i}\right)$. Moreover, indecomposability of $X_{i}$ as a $\Lambda$-module implies indecomposability of $E_{i}$ as an $E$-module, $X_{i} \neq X_{j}$ when $i \neq j$ implies that $E_{i} \neq E_{j}$, and the analogous statements hold for the $F_{i}$. If we can prove that $E_{i} \cong F_{i}$ as $E$-modules it will follow that $X_{i} \cong Y_{i}$ as $\Lambda$-modules. (See, e.g., [GL '88, 1.1] for the proofs of these statements.)

Since localization of finitely presented modules commutes with forming Hom groups, taking the $Q$-localization of decompositions (2.11.1) and repeating the reasoning in the previous paragraph yields a pair of decompositions of left $E_{Q}$-modules:

$$
E_{Q} \cong \bigoplus_{i}\left(\left(E_{i}\right)_{Q}\right)^{e_{i}} \cong \bigoplus_{i}\left(\left(F_{i}\right)_{Q}\right)^{e_{i}}
$$

in which each $\left(E_{i}\right)_{Q}$ is indecomposable, $\left(E_{i}\right)_{Q} \not\left(E_{j}\right)_{Q}$ when $i \neq j$, and the analogous statements hold for the $\left(F_{i}\right)_{Q}$.

Identify the decompositions in (2.11.1) and (2.11.2) with internal direct-sum decompositions of $E$ and $E_{Q}$. Then these decompositions arise by writing the identity element of $E$ in two different ways as a sum of primitive orthogonal idempotents. Let $W$ denote the nilradical of $E$. Since our decompositions of $E$ and $E_{Q}$ arise from orthogonal idempotents, they induce direct-sum decompositions of $E / W$ and $E_{Q} / W_{Q}$. Recall that idempotents can be lifted modulo nil ideals, and the left ideals that they generate are isomorphic modules after the lifting if and only if they were isomorphic before the lifting. Therefore, after replacing $E$ and $E_{Q}$ by $E / W$ and $E_{Q} / W_{Q}$ respectively, we can assume that $E$ is semiprime. [Caution. It will unfold that $E_{Q}$ is semiprime, but this is not yet obvious.]

Since $E$ is semiprime and its $Q$-torsion submodule $T=\operatorname{ker}\left(E \rightarrow E_{Q}\right)$ is a 2-sided ideal that has finite length as an $R$-module hence as an $E$-module, $T$ is generated by a central idempotent of $E$ (see the proof of [GL '89, (1.4.3)]). It follows that every indecomposable left ideal of $E$ is either $Q$-torsion (i.e. its $Q$-localization equals zero) or $Q$-torsionfree. Since each $\left(E_{i}\right)_{Q}$ is nonzero, $E_{i}$ must therefore be $Q$-torsionfree, hence $E$ itself is $Q$-torsionfree. Therefore we can regard $E$ as an $R$-subalgebra of $E_{Q}$. It now follows that $E_{Q}$ is semiprime (otherwise the intersection with $E$ of any nilpotent ideal of $E_{Q}$ would be a nilpotent ideal of $E$ ). In other words, $E_{Q}$ is semisimple artinian ring.

Still identifying our first direct-sum decomposition of $E_{Q}$ with an internal directsum decomposition of $E_{Q}$, we see that the terms $\left(\left(E_{i}\right)^{e_{i}}\right)_{Q}$ become the set of minimal (2-sided) ideals of $E_{Q}$, and the terms $\left(E_{i}\right)^{e_{i}}$ are their intersections with $E$. This proves that each $\left(E_{i}\right)^{e_{i}}=\left(F_{i}\right)^{e_{i}}$ after the above-mentioned identification, and therefore $\left(E_{i}\right)^{e_{i}} \cong\left(F_{i}\right)^{e_{i}}$ before the identification. 
The previous discussion of $e^{\text {th }}$ roots now shows that $E_{i} \cong F_{i}$.

Examples 2.12 (Root summands). Suppose that $R$ is semilocal and $X$ is a f.g. $\Lambda$-module that decomposes like $X_{Q}$.

(i) $X$ can have indecomposable direct summands $U$ such that $U_{Q}$ is not indecomposable.

To see this, let $n$ be any positive integer. Then there is a (noncommutative) integral domain $\Lambda$ that is module-finite over a discrete valuation ring and such that $\Lambda^{n}$ can be written as the direct sum of two indecomposable modules [L '89, 2.4]. On the other hand, since $\Lambda$ is an integral domain, $\Lambda^{n}$ is necessarily decomposes like $\Lambda_{Q}$, and its unique root sumand is $\Lambda$.

(ii) $X$ can have an indecomposable direct summand $P$ such that $P_{Q}$ is also indecomposable, but $P$ is not a root summand of $X$ [i.e. $P$ is not isomorphic to any $X_{i}$ in any decomposition of the form (2.10.1)].

We present the example briefly, using the terminology and machinery in [L '89]. Let $\Gamma$ be a (noncommutative) principal ideal domain that is module-finite and torsionfree over a discrete valuation ring, and such that there is a surjective ring homomorphism $f: \Gamma \rightarrow B=\Delta_{2 \times 2}$, the ring of two-by-two matrices over a division ring $\Delta$ with $H:=\operatorname{ker}(f)=\operatorname{rad}(\Gamma)$, as in [L '89, 2.1]. Let $A$ be the subring of $B$ consisting of diagonal matrices, and let $\Lambda$ be the pullback $\Lambda=\{x \in \Gamma \mid f(x) \in A\}$. Note that $A$ has two indecomposable projective modules, namely $X=\Delta \times 0$ and $Y=0 \times \Delta$. Moreover, an arbitrary f.g. projective $A$-module $X^{a} \oplus Y^{b}$ has the form $P / H P$ for some f.g. projective $\Lambda$-module $P$ if and only if $a+b$ is an even number [L '89, 2.2]; and $P \cong P^{\prime}$ for f.g. projective $\Lambda$-modules $P, P^{\prime}$ if and only if $P / H P \cong P^{\prime} / H P^{\prime}[\mathrm{L} ' 89,1.2(\mathrm{i})]$. Note that $\Lambda / H \cong X \oplus Y$.

It now follows that the integral domain $\Lambda$ has projective modules $P_{1}, P_{2}$ such that $P_{1} / H P_{1} \cong X^{2}$ and $P_{2} / H P_{2} \cong Y^{2}$, and $\Lambda^{2} \cong P_{1} \oplus P_{2}$. Thus $\Lambda^{2}$ decomposes like $\left(\Lambda^{2}\right)_{Q}$ and its unique root summand is $\Lambda$. But since $\Lambda_{Q}$ is a division $R$ algebra, the $P_{i}$ are indecomposable and each $\left(P_{i}\right)_{Q}$ is again indecomposable. In fact, $\left(P_{1}\right)_{Q} \cong \Lambda_{Q} \cong\left(P_{2}\right)_{Q}$. And neither $P_{i}$ is isomorphic to $\Lambda$ because these isomorphisms fail modulo $H$.

We return to the general, non-semilocal $R$. Let $M$ be an $R$-module and $\mathbf{m}$ a maximal ideal of $R$. Then, by Lemma 2.3 we have $\left(M_{\mathbf{m}}\right)_{Q}=\left(M_{\mathbf{m}}\right)_{Q\left(R_{\mathbf{m}}\right)}$, where the unadorned $Q$ denotes $Q(R)$. We take advantage of this notational simplification in what follows.

Lemma 2.13. Let $N, L$ be f.g. $\Lambda$-modules such that every indecomposable direct summand of $N_{Q}$ is isomorphic to a direct summand of $L_{Q}$. Then $(\mathrm{a} \forall \mathbf{m}), N_{\mathbf{m}}$ and $L_{\mathbf{m}}$ decompose like $\left(N_{\mathbf{m}}\right)_{Q}$ and $\left(L_{\mathbf{m}}\right)_{Q}$ respectively, and the root summands of $N_{\mathbf{m}}$ are among those of $L_{\mathbf{m}}$.

Proof. By the Krull-Schmidt theorem for modules of finite length we have $N_{Q} \cong$ $\bigoplus_{i}\left(V_{i}\right)^{e_{i}}$ and $L_{Q} \cong \bigoplus_{i}\left(V_{i}\right)^{d_{i}}$, where the $V_{i}$ are non-isomorphic indecomposable f.g. modules over the artinian ring $\Lambda_{Q}$, each $e_{i} \geq 0$ and each $d_{i}>0$. For each $i$ let $X_{i}$ be a finitely generated $\Lambda$-module such that $\left(X_{i}\right)_{Q} \cong V_{i}$, and let $X=\bigoplus_{i}\left(X_{i}\right)^{e_{i}}$ and $W=\bigoplus_{i}\left(X_{i}\right)^{d_{i}}$. Then $X_{Q} \cong N_{Q}$ and $W_{Q} \cong L_{Q}$. Therefore, (a $\left.\forall \mathbf{m}\right), X_{\mathbf{m}} \cong N_{\mathbf{m}}$ and $W_{\mathbf{m}} \cong L_{\mathbf{m}}$ by Lemma 2.4. To complete the proof we show that, except for finitely many $\mathbf{m}$, the $X_{i}$ provide the desired root summands, that is, $\left(X_{i}\right)_{\mathbf{m}}$ is either zero (and can be ignored), or it is indecomposable and $\left(\left(X_{i}\right)_{\mathbf{m}}\right)_{Q} \cong V_{i}$. 
Let $\mathcal{M}$ be the finite (possibly empty) set of maximal ideals $\mathbf{m}$ such that, when $\mathbf{m} \notin \mathcal{M}$, we have $X_{\mathbf{m}} \cong N_{\mathbf{m}}$ and $W_{\mathbf{m}} \cong L_{\mathbf{m}}$ and both of $X_{\mathbf{m}}$ and $W_{\mathbf{m}}$ are $Q$ torsionfree. Fix any $\mathbf{m} \notin \mathcal{M}$, and any $i$.

Since $V_{i}$ is indecomposable we have $V_{i} \cong\left(V_{i}\right)_{\mathbf{p}}$ for some minimal prime $\mathbf{p}$, by Lemma 2.2. We consider two cases.

Case 1: $\mathbf{m} \nsupseteq \mathbf{p}$. Then $\left(V_{i}\right)_{\mathbf{m}} \cong\left(\left(V_{i}\right)_{\mathbf{p}}\right)_{\mathbf{m}}=0$ by Lemma $2.2\left(\right.$ ii). Since $\left(X_{i}\right)_{\mathbf{m}}$ is $Q$-torsionfree we have $\left(X_{i}\right)_{\mathbf{m}} \subseteq\left(\left(X_{i}\right)_{\mathbf{m}}\right)_{Q} \cong\left(V_{i}\right)_{\mathbf{m}}=0$, and we can therefore ignore $\left(X_{i}\right)_{\mathbf{m}}$.

Case 2: $\mathbf{m} \supseteq \mathbf{p}$. Then $\left(\left(X_{i}\right)_{\mathbf{m}}\right)_{Q} \cong\left(\left(X_{i}\right)_{Q}\right)_{\mathbf{m}} \cong\left(V_{i}\right)_{\mathbf{m}} \cong\left(\left(V_{i}\right)_{\mathbf{p}}\right)_{\mathbf{m}} \cong\left(V_{i}\right)_{\mathbf{p}} \cong V_{i}$ as desired. To see that $\left(X_{i}\right)_{\mathbf{m}}$ is indecomposable, suppose that $\left(X_{i}\right)_{\mathbf{m}}=A \oplus B$ with $A \neq 0$. Since $V_{i}$ is indecomposable and $\left(X_{i}\right)_{\mathbf{m}}$ is $Q$-torsionfree, localization at $Q$ shows that $B_{Q}=0$. Since $\left(X_{i}\right)_{\mathbf{m}}$ is $Q$-torsionfree, so is $B$. Therefore $B=0$.

Remark 2.14. We do not know whether the finite, exceptional set of maximal ideals $\mathbf{m}$ in the previous lemma can be chosen large enough so that the KrullSchmidt theorem holds for decompositions of all remaining $N_{\mathbf{m}}$. As shown in Examples 2.12, uniqueness of the root summands and their multiplicities can be a weaker condition than Krull-Schmidt uniqueness.

However, we now have enough information to show that almost all localizations of f.g. projective $\Lambda$-modules behave as well as free modules over commutative integral domains in two important ways. See Definitions 2.10 for terminology.

Theorem 2.15. Let $P, P^{\prime}$ be a f.g. projective $\Lambda$-modules. Then

(i) $(\mathrm{a} \forall \mathbf{m}) \Lambda_{\mathbf{m}}$ and $P_{\mathbf{m}}$ decompose like $\left(\Lambda_{\mathbf{m}}\right)_{Q}$ and $\left(P_{\mathbf{m}}\right)_{Q}$ respectively, and the root summands of $P_{\mathbf{m}}$ are among those of $\Lambda_{\mathbf{m}}$ (up to isomorphism).

(ii) Suppose that $R$ is semilocal and $P$ and $P^{\prime}$ are direct sums of root summands of $\Lambda$. If $P_{Q} \cong P_{Q}^{\prime}$ then $P \cong P^{\prime}$.

Proof. Statement (i) is a special case of Lemma 2.13. To prove statement (ii), write $P$ and $P^{\prime}$ as direct sums of root summands of $\Lambda$, say

$$
P \cong \bigoplus_{i}\left(X_{i}\right)^{d(i)} \text { and } \quad P^{\prime} \cong \bigoplus_{i}\left(X_{i}\right)^{e(i)}
$$

where, as usual, the $X_{i}$ are pairwise non-isomorphic. It suffices to prove that every $d(i)=e(i)$. Take the $Q$-localization of these decompositions, remembering that root summands remain indecomposable after $Q$-localization, and non-isomorphic root summands of $\Lambda$ remain non-isomorphic after this localization. Since $P_{Q} \cong P_{Q}^{\prime}$ we can apply the Krull-Schmidt theorem for modules of finite length to conclude that every $d(i)=e(i)$, as desired.

Definition 2.16. Return to our basic package deal question: For each $\mathbf{m}$ let $X(\mathbf{m})$ be a f.g. $\Lambda_{\mathbf{m}}$-module. Is there a f.g. $\Lambda$-module $N$ such that $(\forall \mathbf{m}) N_{\mathbf{m}} \cong X(\mathbf{m})$ ? Theorem 2.9 answers the question affirmatively if we already know a $\Lambda$-module $L$ whose localizations $L_{\mathbf{m}}$ we can conveniently compare with the $X(\mathbf{m})$. The statement of the next theorem avoids the necessity of such a global comparison when $\Lambda$ has no nilpotent ideals. Our first step is to isolate the following $Q$-consistency condition that is obviously necessary for the existence of $N$.

Consider a family $\{X(\mathbf{m}) \mid \mathbf{m} \in \operatorname{maxspec}(R)\}$ where each $X(\mathbf{m})$ is a f.g. $\Lambda_{\mathbf{m}^{-}}$ module. We call $\{X(\mathbf{m})\}$ a $Q$-consistent family of f.g. $\left\{\Lambda_{\mathbf{m}}\right\}$-modules if (compare with Remark 2.8):

$$
\mathbf{p} \subseteq \mathbf{m} \cap \mathbf{n} \Rightarrow X(\mathbf{m})_{\mathbf{p}} \cong X(\mathbf{n})_{\mathbf{p}}
$$


for $\mathbf{p} \in \operatorname{minspec}(R)$ and $\mathbf{m}, \mathbf{n} \in \operatorname{maxspec}(R)$. For each $\mathbf{p} \in \operatorname{minspec}(R)$ choose a maximal ideal $\mathbf{m}(\mathbf{p}) \supseteq \mathbf{p}$ and then set

$$
X(Q)=\bigoplus_{\mathbf{p} \in \operatorname{minspec}(R)} X(\mathbf{m}(\mathbf{p}))_{\mathbf{p}}
$$

Because of $Q$-consistency, the isomorphism class of the $\Lambda_{Q}$-module $X(Q)$ is independent of the particular choice of maximal ideals $\mathbf{m}(\mathbf{p})$. Moreover, if the desired $\Lambda$-module $N$ exists, we necessarily have $N_{Q} \cong X(Q)$.

We now answer the package deal question for localizations of modules,as posed in Definition 2.16, if certain $\Lambda_{Q}$-modules are projective. This hypothesis is always satisfied if the ring $\Lambda$ is semiprime, for then the ring $\Lambda_{Q}$ is semisimple artinian and therefore all of its modules are projective. See Definitions 2.10 for additional terminology.

Theorem 2.17. Let $\{X(\mathbf{m}): \mathbf{m} \in \operatorname{maxspec}(R)\}$ be a $Q$-consistent family of f.g. $\left\{\Lambda_{\mathbf{m}}\right\}$-modules, and suppose that every $X(\mathbf{m})_{Q}$ is a projective $\Lambda_{Q}$-module. Then the following statements are equivalent.

(i) There is a f.g. $\Lambda$-module $N$ such that $N_{\mathbf{m}} \cong X(\mathbf{m})(\forall \mathbf{m})$.

(ii) $(a \forall \mathbf{m})$ such that $\Lambda_{\mathbf{m}}$ decomposes like $\Lambda_{Q}, X(\mathbf{m})$ is a direct sum of root summands of $\Lambda_{\mathrm{m}}$.

Proof. Both implications use the fact that, since the Krull-Schmidt theorem holds for f.g. modules over the artinian ring $\Lambda_{Q}$, every indecomposable f.g. projective $\Lambda_{Q}$-module is isomorphic to a direct summand of $\Lambda_{Q}$.

(i) $\Rightarrow$ (ii). Let $L=\Lambda$, considered as a left $\Lambda$-module. In view of the preceeding paragraph every indecomposable direct summand of $N_{Q}$ is isomorphic to a direct summand of $L_{Q}$. Therefore the implication (i) $\Rightarrow$ (ii) is a special case of Lemma 2.13 .

(ii) $\Rightarrow$ (i). We first find a module $M$ that has the required localizations almost everywhere, and then change the finite number of remaining localizations. Because of the $Q$-consistency hypothesis we can form the $\Lambda_{Q}$-module $X(Q)$ defined in Definition 2.16. Let $M$ be the $\Lambda$-submodule of $X(Q)$ generated by some finite set of $\Lambda_{Q}$-generators of $X(Q)$. Then $M_{Q} \cong X(Q)$ and $M_{Q}$ is a projective $\Lambda_{Q}$-module.

By the first paragraph of this proof, every indecomposable direct summand of the projective $\Lambda_{Q}$-module $M_{Q}$ is isomorphic to a direct summand of $\Lambda_{Q}$. Therefore we can apply Lemma 2.13 to the pair of modules $M, \Lambda$. We conclude that $(\mathrm{a} \forall \mathbf{m}) M_{\mathbf{m}}$ and $\Lambda_{\mathbf{m}}$ decompose like $\left(M_{\mathbf{m}}\right)_{Q}$ and $\left(\Lambda_{\mathbf{m}}\right)_{Q}$ respectively, and the root summands of $M_{\mathrm{m}}$ are among those of $\Lambda_{\mathrm{m}}$.

We claim that $M_{\mathbf{m}} \cong X(\mathbf{m})(\mathrm{a} \forall \mathbf{m})$. Consider any $\mathbf{m}$ that satisfies the conditions in the final sentence of the previous paragraph. After discarding finitely many more $\mathbf{m}$ we have that $X(\mathbf{m})$ is a direct sum of root summands of $\Lambda_{\mathbf{m}}$. By Theorem 2.15(ii) it now suffices to check that $\left(M_{\mathbf{m}}\right)_{Q} \cong X(\mathbf{m})_{Q}$. Since $M_{Q} \cong X(Q)$ it suffices to prove $X(Q)_{\mathbf{m}} \cong X(\mathbf{m})_{Q}$, which, with the help of Lemma 2.2, follows easily from the definition of $X(Q)$.

Now that the claim is proved, we obtain the desired module $N$ by applying the package deal theorem for localizations of modules 2.9 to change the remaining finite number of localizations $M_{\mathbf{m}}$ to $X(\mathbf{m})$. This is possible since, as already shown, $\left(M_{\mathbf{m}}\right)_{Q} \cong X(\mathbf{m})_{Q}$. 
The following special case of Theorem 2.17 is particularly easy to understand, and extends a result mention in the introduction to this paper.

Corollary 2.18. Assume that $R=\Lambda$ and $\Lambda$ has no nilpotent elements. For every $\mathbf{m} \in \operatorname{maxspec}(R)$ let $X(\mathbf{m})$ be a f.g. $\Lambda_{\mathbf{m}}$-module. Then there exists a f.g. $\Lambda$-module $N$ such that $N_{\mathbf{m}} \cong X(\mathbf{m})(\forall \mathbf{m})$ if and only if

(i) $X(\mathbf{m})$ is a free $\Lambda_{\mathbf{m}}$-module $(\mathbf{a} \forall \mathbf{m})$; and

(ii) $Q$-consistency condition (2.16.1) holds.

Proof. If. By hypothesis (ii) the $X(\mathbf{m})$ form a $Q$-consistent family in the sense of Definition 2.16. By hypothesis (i) $X(\mathbf{m})$ is free for almost all $\mathbf{m}$ such that $\Lambda_{\mathbf{m}}$ decomposes like $\left(\Lambda_{\mathbf{m}}\right)_{Q}$. For such $\mathbf{m}, X(\mathbf{m})$ is clearly a direct sum of root summands of $\Lambda_{\mathbf{m}}$ (namely $\Lambda_{\mathbf{m}}$ itself). Therefore $N$ exists by Theorem 2.17.

Only if. Since $N$ exists, the family $\{X(\mathbf{m})\}$ is $Q$-consistent, that is, condition (ii) holds. By Theorem $2.15 \Lambda_{\mathbf{m}}$ decomposes like $\left(\Lambda_{\mathbf{m}}\right)_{Q}(\mathrm{a} \forall \mathbf{m})$. By Theorem 2.17 $X(\mathbf{m})$ is a direct sum of root summands of $\Lambda_{\mathbf{m}}$ for almost all of the $\mathbf{m}$ in the previous sentence, hence for almost $\mathbf{m}$. In particular, $X(\mathbf{m})$ is projective over the local ring $\Lambda_{\mathrm{m}}=R_{\mathrm{m}}$ and is therefore free, as desired.

Example 2.19. The preceeding corollary has some content even in the following very simple situation. Assume that $R=\Lambda$ is an integral domain with infinitely many maximal ideals $\mathbf{m}$ and no $\Lambda_{\mathbf{m}}$ is a discrete valuation ring. Thus every localization $\Lambda_{\mathbf{m}}$ has nonzero ideals not isomorphic to $\Lambda_{\mathbf{m}}$ as $\Lambda_{\mathbf{m}}$-modules. However, the preceding corollary (with $\Lambda=R$ ) shows that a family $\{X(\mathbf{m}): \mathbf{m} \in \operatorname{maxspec}(R)\}$ of nonzero ideals $X(\mathbf{m})$ of $R_{\mathbf{m}}$ is isomorphic to the set of localizations of a nonzero ideal of $R$ if and only if $X(\mathbf{m}) \cong R_{\mathbf{m}}(\mathrm{a} \forall \mathbf{m})$. (Note that M. Hochster has given an example of such a domain [H '73].)

Our final main result in this section is that indecomposability of $\Lambda$-modules is semilocally determined.

Theorem 2.20. Let $M$ be an indecomposable f.g. $\Lambda$-module. Then there is a finite set $\mathcal{M}$ of maximal ideals of $R$ such that $M_{\mathcal{M}}$ is an indecomposable $\Lambda_{\mathcal{M}}$-module, and remains indecomposable if $\mathcal{M}$ is replaced by any larger finite set of maximal ideals.

Proof. $(a \forall \mathbf{m}) M_{\mathbf{m}}$ decomposes like $\left(M_{\mathbf{m}}\right)_{Q}$, by Lemma 2.13 . Let $\mathcal{M}$ be the finite set of $\mathbf{m}$ for which this is not true, and enlarge $\mathcal{M}$ if necessary (keeping it finite) so that so that $\mathcal{M}$ covers minspec $(R)$, and perhaps contains additional maximal ideals. We claim that, for any such $\mathcal{M}, M_{\mathcal{M}}$ is indecomposable.

Let $X$ be a $\Lambda_{\mathcal{M}}$-module such that $X \mid M_{\mathcal{M}}$ (i.e. $X$ is isomorphic to a direct summand of $M_{\mathcal{M}}$ ). We want to show that $X=0$ or $X=M_{\mathcal{M}}$ (i.e. the image of $X$ in $M_{\mathcal{M}}$ is $M_{\mathcal{M}}$ itself). We have $X \cong N_{\mathcal{M}}$ for some f.g. $\Lambda$-module $N$. Then $N_{\mathbf{m}} \mid M_{\mathbf{m}}(\forall \mathbf{m} \in \mathcal{M})$. We claim that $N_{\mathbf{m}} \mid M_{\mathbf{m}}$ also holds for almost all $\mathbf{m} \notin \mathcal{M}$.

Since $\mathcal{M}$ covers minspec $(R)$, we have $N_{\mathbf{p}} \mid M_{\mathbf{p}}$ for every minimal prime of $R$, and hence, $N_{Q} \mid M_{Q}$ by Lemma 2.2. Therefore $N_{\mathbf{m}} \mid M_{\mathbf{m}}(\mathrm{a} \forall \mathbf{m})$ by Lemma 2.4(iii), as claimed.

We now wish to change the finite number of remaining localizations $N_{\mathbf{m}}$ to achieve $N_{\mathbf{m}} \mid M_{\mathbf{m}}$ there, too. Recall that $M_{\mathbf{m}}$ decomposes like $\left(M_{\mathbf{m}}\right)_{Q}$ at all these localizations. The package deal theorem for localizations of modules 2.9 therefore allows us to make the needed changes: Change $N_{\mathbf{m}}$ by a direct sum of root summands of $M_{\mathbf{m}}$, choosing whatever number of root summands are needed so as not to change the isomorphism class of $\left(N_{\mathbf{m}}\right)_{Q}$. 
[GL '89, 4.1] states: If $N$ and $M$ are f.g. $\Lambda$-modules such that $N_{\mathbf{m}} \mid M_{\mathbf{m}}$ for every maximal ideal $\mathbf{m}$, then $N^{\prime} \mid M$ for some $N^{\prime}$ such that $N_{\mathbf{m}}^{\prime} \cong N_{\mathbf{m}}(\forall \mathbf{m})$. Since $M$ is indecomposable, we have either $N^{\prime}=0$ (in which case $0=N_{\mathcal{M}}^{\prime} \cong N_{\mathcal{M}}=X$ ) or $N^{\prime}=M$ (in which case $X=M_{\mathcal{M}}$ because this is true locally).

\section{PaCkages of Completions}

In this section we determine when a f.g. $\hat{\Lambda}$-module is the completion of some f.g. $\Lambda$-module. We assume that $R$ is semilocal (and, as always, noetherian of dimension 1 ) and $\Lambda$ denotes any module-finite $R$-algebra.

Definitions, Notation 3.1 (Completions). Let $J=J(R)$, the Jacobson radical of $R$. We denote the $J$-adic completion of $R$ by $\hat{R}$ and the $J$-adic completion of any f.g. $R$-module $M$ by $\hat{M}$. Since $M$ is f.g. and $R$ is noetherian and semilocal, the Krull intersection theorem shows that $M \subseteq \hat{M}$ [B '72, Chap. III, §3.3, Prop. 6]. In addition, $\hat{R}$ is a faithfully flat $R$-module, $\hat{M}=\hat{R} M$, and the canonical map $\hat{R} \otimes_{R} M \rightarrow \hat{M}$ is a bijection [B '72, Chap. III, $\S 3.4$, Thm. 3 and Cor. 1, and $\S 3.5$, Prop. 9]. We regard this bijection as equality $\hat{R} \otimes_{R} M=\hat{R} M=\hat{M}$ whenever convenient. In particular, $\hat{M}$ is a f.g. $\hat{R}$-module.

Since $\Lambda$ is a module-finite $R$-algebra the foregoing discussion shows that $\hat{\Lambda}=$ $\hat{R} \otimes_{R} \Lambda$; and if $M$ is any f.g. $\Lambda$-module we have $\hat{M}=\hat{R} \otimes_{R} M$, a f.g. $\hat{\Lambda}$-module.

Caution. $\hat{R}$ has nilpotent elements whenever the integral closure of $R$ in its total quotient ring is not a f.g. $R$-module, even if $R$ itself has no nilpotent elements [Mtl '73, 7.1]. Nevertheless, the crucial properties that make completions useful to us still hold: (i) the Krull-Schmidt theorem holds for f.g. $\hat{\Lambda}$-modules [CR '81, (6.12)]; and (ii) for f.g. $\Lambda$-modules $M$ and $N$ we have $M \cong N$ as $\Lambda$-modules if and only if $\hat{M} \cong \hat{N}$ as $\hat{\Lambda}$-modules. (Statement (ii) is a special case of Grothendieck's theorem on isomorphism by faithfully flat descent over semilocal rings [G '65, 2.5.8]).

Let $\hat{R}_{Q}=\hat{R} \otimes_{R} R_{Q}$; that is, let $\hat{R}_{Q}$ be the $Q(R)$-localization of $\hat{R}$. We also call $\hat{R}_{Q}$ the $J(R)$-induced completion of $R_{Q}$ (but see the Caution statement below). We claim:

$$
\hat{R}_{Q} \text { is an artinian ring. }
$$

$\hat{R}$ is noetherian of Krull dimension 1 [N'62, (17.2)], and its maximal ideals are the ideals $\hat{R} \mathbf{m}$, where $\mathbf{m}$ runs through the maximal ideals of $R$ [Mts '80, p. 173]. Since $\mathbf{m}_{Q}=R_{Q}$ for every maximal ideal $\mathbf{m}$ of $R$ that is not a minimal prime, the preceding sentence shows that all prime ideals of $\hat{R}_{Q}=(\hat{R})_{Q}$ are minimal primes, proving the claim.

Caution. As mentioned in the introduction to this paper, the $J(R)$-induced completion $\hat{R}_{Q}$ is not an "adic" completion of $R_{Q}$ with respect to some ideal of $R_{Q}$. But it is the completion of $R_{Q}$ with respect to a suitable topology determined by $J(R)$, as explainded in Remark 3.6. However, what really interests us is the flatness properties of completions, and these can be obtained more quickly by ignoring the topology and proceeding as explained below.

Since $\hat{R}$ is a faithfully flat $R$-module, it is easy to see that $\hat{R}_{Q}$ is a faithfully flat $R_{Q}$-module.

For a f.g. $\Lambda_{Q}$-module $V$, set $\hat{V}=\hat{R} \otimes_{R} V$ and call it the $J(R)$-induced completion of $V$. Note that, by slight abuse of notation, the notation $\hat{X}$ now has two meanings: the $J(R)$-adic completion $\hat{X}=\hat{R} \otimes_{R} X$ if $X$ is a f.g. $\Lambda$-module, and the $J(R)$-induced 
completion $\hat{X}=\hat{R} \otimes_{R} X$ if $X$ is a f.g. $\Lambda_{Q}$-module. We shall never have any occasion to refer to $\hat{R} \otimes_{R} X$ for an arbitrary non-f.g. $\Lambda$-module $X$.

Note that $\hat{\Lambda}_{Q}=R_{Q} \otimes_{R} \hat{R} \otimes_{R} \Lambda$ is a module-finite $\hat{R}_{Q}$-algebra; and $\hat{V}$ is a f.g. $\hat{\Lambda}_{Q}$-module when $V$ is a f.g. $\Lambda_{Q}$-module. Since $\hat{\Lambda}_{Q}$ is a module-finite $\hat{R}_{Q}$-algebra, statement (3.1.1) shows:

$$
\hat{\Lambda}_{Q} \text { is an artinian ring. }
$$

Since $\hat{R}$ is a flat $R$-module, we have $V \subseteq \hat{V}$ via $v \rightarrow 1 \otimes v$ [B '72, Chap. I, $\S 3.5$, Prop. 9]. We always make this identification. One consequence is that $\hat{R} V=\hat{V}$. Another consequence of this identification is that, if $M$ is a $\Lambda$-submodule of $V$, we can also regard it as a $\Lambda$-submodule of $\hat{V}$. Therefore $\hat{R} M \cap V$ is a well-defined $\Lambda$-submodule of $V$ and clearly contains $M$. Since $\hat{R}$ is a faithfully flat $\hat{R}$-module, we have the following "up-down" result [B '72, Chap. I, §3.5, Prop. 10(ii)].

Lemma 3.2. Let $V$ be a f.g. $\Lambda_{Q}$-module, and $M$ a $\Lambda$-submodule of $V$. Then $(\hat{R} M) \cap V=M$ (intersection in $\hat{R} V=\hat{V})$.

The corresponding "down-up" result is false; and this is the reason that we need a package deal theorem for completions. We begin with the following key situation.

Lemma 3.3. Let $V$ be a f.g. $\Lambda_{Q}$-module, and $W$ a f.g. $\hat{\Lambda}$-submodule of $\hat{V}$ such that $W_{Q}=\hat{V}$. Then $W \cap V$ is a f.g. $\Lambda$-module such that $\hat{R}(W \cap V)=W$ and $(W \cap V)_{Q}=V$. Moreover, $W$ is a $J$-adic completion of $W \cap V$.

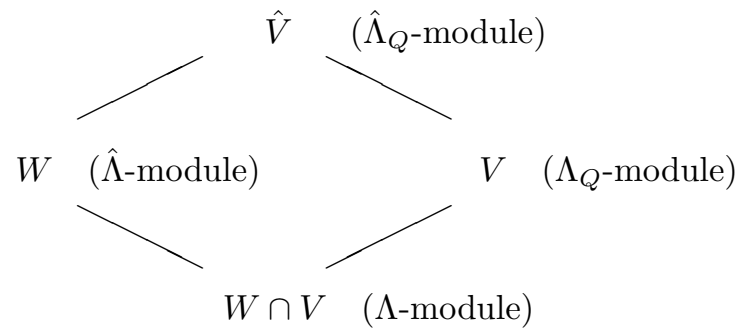

Proof. Let $L$ be the $\Lambda$-module generated by some finite set of $\Lambda_{Q}$ generators of $V$. Then $L_{Q}=V$, and hence $\hat{L}_{Q}=\hat{V}$. Since $\hat{L}$ and $W$ are $\hat{\Lambda}$-f.g. and $\hat{L}_{Q}=\hat{V}=W_{Q}$, there exist $c, d \in R-\bigcup Q$ such that $d W \subseteq \hat{L}$ and $c \hat{L} \subseteq d W$. After replacing $L$ by $d^{-1} L$ we have $c \hat{L} \subseteq W \subseteq \hat{L}$. Using Lemma 3.2 and recalling that $\hat{L}=\hat{R} L$ yields

$$
c L \subseteq W \cap V \subseteq \hat{L} \cap V=L \quad[\subseteq V] .
$$

Since $L$ is a noetherian $\Lambda$-module we see that $W \cap V$ is a f.g. $\Lambda$-module; and localizing (3.3.2) at $Q$ shows that $(W \cap V)_{Q}=V$.

Next we show that $W=\hat{R}(W \cap V)$. But since the $R$-algebra $\hat{R}$ is a faithfully flat $R$-module we have $\hat{R}(W \cap V)=\hat{R} W \cap \hat{R} V=W \cap \hat{V}=W$ [B '72, Chap. I, $\S 3.5$, Prop. 10].

In preparation for the proof of the final assertion of the lemma we note that the following natural map is one-to-one since $\hat{R}$ is a flat $R$-module:

$$
\hat{R} \otimes_{R}(W \cap V) \longrightarrow \hat{R} \otimes_{R} V=\hat{V} .
$$


Now we prove that $W$ is a $J$-adic completion of $W \cap V$. It suffices to prove that the natural surjection $\hat{R} \otimes_{R}(W \cap V) \rightarrow \hat{R}(W \cap V)=W$ is an isomorphism. But any element of the kernel of this map is in the kernel of the monomorphism of the map in (3.3.3), and therefore equals zero as desired.

Package Deal Theorem 3.4 (Completions). The following conditions are equivalent for a f.g. $\hat{\Lambda}$-module $W$.

(i) $W \cong \hat{M}$ for some f.g. $\Lambda$-module $M$.

(ii) $W_{Q} \cong \hat{V}$ for some f.g. $\Lambda_{Q}$-module $V$.

Proof. To prove the nontrivial implication, assume (ii) holds. First we consider the $Q$-torsionfree case [the canonical map $W \rightarrow W_{Q}$ is one-to-one]. Hence we can suppose $W \subseteq W_{Q}=\hat{V}$. Then, by Lemma 3.2 we have that $W$ is a $J$-adic completion of the f.g. $\Lambda$-module $W \cap V$.

Next we consider the $Q$-torsion case, $W_{Q}=0$. In this case $d W=0$ for some $d \in R-\bigcup Q$. So $W$ is a finitely generated module over the artinian $\operatorname{ring} R / d R \cong$ $(R / d R)^{\wedge} \cong \hat{R} / d \hat{R}$. Therefore $W$ has finite length as an $R$-module and hence is the $J$-adic completion of itself.

Now we put the torsion and torsionfree cases together. Consider the short exact sequence

$$
\mathcal{E}: \quad T \hookrightarrow W \rightarrow X
$$

where $X$ is the image of $W$ under the canonical map $W \rightarrow W_{Q}$ and $T=$ $\operatorname{ker}(W \rightarrow X)=\operatorname{ker}\left(W \rightarrow W_{Q}\right)$ is the $Q$-torsion submodule of $W$.

Since $T_{Q}=0$, localizing $\mathcal{E}$ at $Q$ shows that $X_{Q} \cong W_{Q} \cong \hat{V}$, so the torsionfree case shows that $X \cong \hat{N}$ for some f.g. $\Lambda$-module $N$. The torsion case shows that $T=\hat{T}$.

Localizing $\operatorname{Ext}_{\Lambda}^{1}(N, T)$ at $Q$, and using the fact that $T_{Q}=0$ shows that $\operatorname{Ext}_{\Lambda}^{1}(N, T)_{Q}=0$. So $\operatorname{Ext}_{\Lambda}^{1}(N, T)$, like $T$, is an $R$-module of finite length and therefore equals its own $J$-adic completion. Thus, after making the identification $X=\hat{N}$, we have the canonical isomorphisms

$$
\operatorname{Ext}_{\Lambda}^{1}(N, T)=\left[\operatorname{Ext}_{\Lambda}^{1}(N, T)\right]^{\wedge}=\operatorname{Ext}_{\hat{\Lambda}}^{1}(\hat{N}, \hat{T})=\operatorname{Ext}_{\hat{\Lambda}}^{1}(X, T) .
$$

Let $\mathcal{D}$ be the element of $\operatorname{Ext}_{\Lambda}^{1}(N, T)$ that is carried to $\mathcal{E}$ by the isomorphisms in (3.4.1). Then the middle term of $\mathcal{D}$ is a f.g. $\Lambda$-module $M$ such that $\hat{M} \cong W$.

Remark 3.5. (i) Let $W$ be a f.g. $\hat{\Lambda}$-module and let $t(W)=\operatorname{ker}\left(W \rightarrow W_{Q}\right)$, the $Q$ torsion submodule of $W$. An immediate corollary of the preceding theorem is that $W$ is the completion of some $\Lambda$-module if and only if the $Q$-torsionfree $\hat{\Lambda}$-module $W / t(W)$ is the completion of some $\Lambda$-module [since $t(W)_{Q}=0$ ].

(ii) In order to use our package deal theorem we need a convenient way of verifying condition (ii). Thus it would be helpful to have an easily-described family of $\Lambda$-modules $P$ such that, for every $\hat{\Lambda}_{Q}$-module $V, V$ is the completion of some $\Lambda_{Q}$-module if and only if $V \cong \hat{P}_{Q}$ for some $P$ in the set. In integral representation theory, the modules $P$ that play this role are the projective modules over some maximal order containing $\Lambda$. In Section 6 we extend this to orders $\Lambda$ that are not contained in a maximal order, by showing that the f.g. projective modules $P$ over any convenient splitting order, or any normalization of $\Lambda$, can be used for this purpose. 
We limit our investigation of this type of question to the situation that $\Lambda$ is an $R$-order in a semisimple artinian ring, although the preceding package deal theorem does not make such a restriction. See Theorem 6.15.

Remark 3.6 (Topological version of $\hat{V}$ ). Let $V$ be a f.g. $\Lambda_{Q}$-module. It is possible to view the $J(R)$-induced completion $\hat{V}$ as a topological completion as follows. Choose a f.g. $\Lambda$-submodule of $L \subseteq V$ such that $L_{Q}=V$. Then the family of $\Lambda$-submodules $\left\{J(R)^{n} L\right\}$ of $V$ has intersection zero; so forming Cauchy sequences with respect to this filtration yields a Hausdorff topology on $V$. It can be verified that this topology, hence the resulting completion of $V$, is independent of the choice of $L$; and this completion is canonically isomorphic to $\hat{V}=\hat{R} \otimes_{R} V$, as defined in Notation 3.1. See [B '72, III, $\S \S 2.1,2.6]$ for the necessary machinery, but not the actual result. This procedure generalizes the familiar completion of an $F$-vector space with respect to a discrete valuation of the field $F$, when $F=R_{Q}$ for some discrete valuation ring $R$. See [R'75].

We close this section by extending two simple results (needed later, and familiar in the settings of commutative algebra and integral representation theory) to our situation.

Lemma 3.7. Every $\hat{\Lambda}$-module of finite length has finite length as an $R$-module (not just as an $\hat{R}$-module), and its $R$-submodules coincide with its $\hat{R}$-submodules.

Proof. Every $\hat{\Lambda}$-module of finite length has finite length as an $\hat{R}$-module (Lemma 1.6, applied to the $\hat{R}$-algebra $\hat{\Lambda})$. Also, the canonical isomorphism $\hat{R} / J(\hat{R})=R / J$ shows that every $\hat{R}$-module of finite length has the same length as an $R$-module. This proves the first assertion of the lemma.

For the second assertion let $M$ be an $R$-submodule of some $\hat{R}$-module $W$ of finite length. Then we have $M \subseteq \hat{R} M \subseteq W$. By the previous paragraph $W$ has finite $R$-length and we therefore have $\lambda(M) \leq \lambda(\hat{R} M)$, where $\lambda(\ldots)$ denotes $R$-length. It now suffices to prove the opposite inequality. Note that every $R$-module of finite length is its own $J$-adic completion. Since $\hat{M}=\hat{R} \otimes M$ maps onto the submodule $\hat{R} M$ of $X$ we therefore have $\lambda(M)=\lambda(\hat{M}) \geq \lambda(\hat{R} M)$, as desired.

Proposition 3.8. Let $M$ be a f.g. $\Lambda$-module. Then the set of $\Lambda$-submodules $N \subseteq M$ such that $M / N$ has finite length is in one-to-one correspondence with the set of $\hat{\Lambda}$ submodules $X \subseteq \hat{M}$ such that $\hat{M} / X$ has finite $\hat{\Lambda}$-length via

$$
N \rightarrow X=\hat{R} N \quad \text { and } \quad X \rightarrow N=X \cap M .
$$

Moreover, $\hat{M} / X \cong M / N$ as $\Lambda$-modules.

Proof. We have $N=\hat{R} N \cap M$ for all submodules of $M$ by Lemma 3.2. Since $M / N$ has finite $\Lambda$-length, it has finite $R$-length by Lemma 1.6 , and is therefore its own $J$-adic completion. Hence $M / N \cong \hat{R} \otimes M / N \cong \hat{M} / \hat{R} N$, as claimed.

Conversely, suppose that $\hat{M} / X$ has finite $\hat{\Lambda}$-length. We need to prove that $\hat{R}(X \cap M)=X$. By Lemma 3.7 the $\hat{\Lambda}$-module $\hat{M} / X$ of finite length has finite $R$-length, and is therefore annihilated by some power $J^{s}$ of $J=J(R)$. Hence $X \supseteq J^{s} \hat{M}$. As in the previous paragraph the completion functor $M \rightarrow \hat{M}$ induces an isomorphism $M / J^{s} M \cong \hat{M} / J^{s} \hat{M}$, which we regard as equality. Then $X / J^{s} \hat{M}=$ $N / J^{s} M$ for some $\Lambda$-submodule $N\left(J^{s} M \subseteq N \subseteq M\right)$ such that $\hat{R} N=X$. Therefore $N=\hat{R} N \cap M=X \cap M$ by Lemma 3.2. Therefore $\hat{R}(X \cap M)=\hat{R} N=X$, as desired. 


\section{Normalizations}

In this section we assume that $R$ and $Z(\Lambda)$ (not necessarily semilocal) have no artinian ring-direct summands and no nonzero nilpotent ideals; and $\Lambda$ denotes an $R$-order in the semisimple artinian ring $\Lambda_{Q}$, as defined in (1.1.1). In this situation $R_{Q}$ becomes the total quotient ring of $R$, and $R$ itself is a $Q$-torsionfree $R$-module. As mentioned in Notation 1.1, we therefore use the more traditional terminology torsionfree module instead of " $Q$-torsionfree module".

This section surveys the basic properties of noncommutative normalizations and our critical link with integral representation theory, the relation between normalizations of $R$-orders and maximal orders over the normalization of $R$.

Definitions, Notation 4.1. Throughout the rest of this paper $\Gamma$ denotes a normalization of $\Lambda$; that is, any maximal element $\Gamma$ of the family of rings such that $\Lambda \subseteq \Gamma \subseteq \Lambda_{Q}$ and $\Gamma$ is integral over $R$. Such a $\Gamma$ obviously exists, by Zorn's Lemma. Note that the property that $\Gamma$ is a normalization of $\Lambda$ is a ring-theoretic property of the inclusion $\Lambda \subseteq \Gamma$. That is, it does not depend upon the particular ring $R$ over which $\Lambda$ is a module-finite algebra, because an element of $\Gamma$ is integral over $R$ if and only if it is integral over the center $Z(\Lambda)$.

(4.1.1) Every f.g. $R$-subalgebra of $\Gamma$ is a f.g. $R$-module.

Since every element of $\Gamma$ is integral over $R$, this is a special case of Shirshov's theorem [MR '87, 13.8.8].

We now summarize the link with integral representation theory that is provided by normalizations.

Let $\tilde{R}$ denote the integral closure of $R \cdot 1_{\Lambda}$ in $Z\left(\Lambda_{Q}\right)$, which equals the integral closure of $Z(\Lambda)$ in $Z\left(\Lambda_{Q}\right)$. We claim that $\tilde{R}$ is a direct sum of finitely many Dedekind domains $\tilde{R}_{k}$, and none of these Dedekind domains is a field.

Proof. Since $Z(\Lambda)$ has no nilpotent elements, $Z\left(\Lambda_{Q}\right)$ is a finite direct sum of fields $F_{k}$ and the ideals $\mathbf{p}_{k}=\operatorname{ker}\left(Z(\Lambda) \rightarrow F_{k}\right)$ are the minimal prime ideals of $Z(\Lambda)$. Since $Z(\Lambda)$ has no artinian direct summands, Lemma 1.3 shows that no minimal prime ideal of $Z(\Lambda)$ is a maximal ideal. This shows that, for each $k$, the zero ideal of $S_{k}$ is not a maximal ideal. Hence $S_{k}$ has dimension exactly one; in particular it is not a field. Since $S_{k}$ is noetherian of dimension 1, the Krull-Akizuki theorem [N '62, (33.2)] shows that its normalization $\tilde{R}_{k}$ is again noetherian of dimension one, hence a Dedekind domain. Since $\tilde{R}=\bigoplus_{k} \tilde{R}_{k}$, the claim is proved.

(4.1.2) $\tilde{R}=Z(\Gamma)$, and $\Gamma$ is a maximal $\tilde{R}$-order in $\Lambda_{Q}$.

(4.1.3) There is a (finite) decomposition $\Gamma=\bigoplus_{k} \Gamma_{k}$ where each ring $\Gamma_{k}$ is a central maximal order over a Dedekind domain (one of the direct summands of $\tilde{R}$ ) in one of the simple artinian ring-direct summands of $\Gamma_{Q}$.

(4.1.4) Every maximal $\tilde{R}$-order in $\Lambda_{Q}$ containing $\Lambda$ is a normalization of $\Lambda$.

Statements (4.1.2)-(4.1.4) are proved in [GL '89, 2.5]. However, the proof given there omitted mention of Shirshov's theorem (4.1.1).

Note that (4.1.3) guarantees that normalizations have the familiar ring-theoretic properties of maximal orders. For example, all left ideals are projective modules [R '75, (21.4)], and a left ideal $L$ is indecomposable if and only if $L_{Q}$ is a simple $\Lambda_{Q}$-module [R ' $\left.75,(21.5)\right]$. We make frequent use of the following part of statement 
(4.1.2):

$\Gamma$ is a f.g. $\tilde{R}$-module.

(4.1.6) The sets of maximal (2-sided) ideals of the rings $\Gamma$ and $\tilde{R}$ are in one-to-one correspondence via contraction; that is, via $A \rightarrow(A \cap \tilde{R})\left[\mathrm{R}^{\prime} 75,(22.4)\right]$.

(4.1.7) Let $\Gamma^{\prime}$ be any other normalization of $\Lambda$. Then $\Gamma$ and $\Gamma^{\prime}$ are Morita equivalent. Indeed, there is an invertible $\left(\Gamma, \Gamma^{\prime}\right)$-subbimodule $M$ of $\Lambda_{Q}$ such that $\Gamma^{\prime}=$ $H^{-1} \Gamma H$. In particular, if $R$ is semilocal, any two normalizations of $\Lambda$ are conjugate in $\Lambda_{Q}$.

To prove (4.1.7) let $\Lambda_{k}$ be the projection of $\Lambda$ in the simple ring-direct summand $\left(\Gamma_{k}\right)_{Q}$ of $\Lambda_{Q}$. In view of (4.1.2) every normalization of $\Lambda$ is also a normalization of $\bigoplus_{k} \Lambda_{k}$. Therefore, to prove (4.1.7), we can work one coordinate ring $\Lambda_{k}$ at a time. In this situation the desired result is proved in [R '75, (22.21)]. When $R$ is semilocal, so is $\tilde{R}$, by Lemma 4.2 below. Therefore the invertible bimodule $M$ becomes principal (on each side), yielding the claimed conjugacy.

(4.1.8) If $R$ is semilocal, then each $\Gamma_{k}$ is a full matrix ring (of size usually denoted by $n_{k} \times n_{k}$ ) over a semilocal principal ideal domain (possibly noncommutative). Hence the Krull-Schmidt theorem holds for f.g. $\Gamma$-modules.

As already mentioned, $\tilde{R}$ is semilocal when $R$ is. Now see the proofs of [R ${ }^{7} 75$, (18.7) and (21.6)], noting that $N$ in (21.6) is free in the semilocal case.

(4.1.9) (i) Every $\Gamma$-module of finite length has finite length as an $R$-module.

(ii) For every regular element $c$ of $Z(\Lambda), \Gamma / c \Gamma$ has finite length as an $R$-module.

(iii) $J(Z(\Lambda)) \subseteq J(\Gamma)$.

We prove (4.1.9) by reducing to the situation that $\Gamma$ is module-finite over $R$, using the part of our link with integral representation theory that states that $\Gamma$ is a module-finite $\tilde{R}$-algebra [see (4.1.2)].

(i). Since $\Gamma$ is a module-finite $\tilde{R}$-algebra, the given $\Gamma$-module of finite length has finite length as an $\tilde{R}$-module [Lemma 1.6]. It therefore suffices to show that every simple $\tilde{R}$-module $\tilde{R} / A$ has finite length as an $R$-module. If $R$ and hence $\tilde{R}$ are integral domains, this is part of the conclusion of the Krull-Akizuki theorem [N '62, (33.2)]. Since $R$ has no nilpotent elements, the general case is easily reduced to this.

(ii). By statement (i) it suffices to show that $\Gamma / \Gamma c$ is an artinian ring. Since $R$ is now out of the picture, we can redefine $R$ to be $Z(\Gamma)$, so that $\Gamma$ becomes modulefinite over $R$ by (4.1.2). Since $c$ is a regular element of $R$, and $R$ has dimension 1 , the ring $R / c R$ is artinian. Therefore the module-finite $R / c R$-algebra $\Gamma / \Gamma c$ is an artinian ring, as desired.

(iii). We may take $R=Z(\Lambda)$. Since $\tilde{R}=Z(\Gamma)$ is an integral extension of $R$, the lying-over and going-up theorems of commutative algebra [K '70, Theorem 44] show that every maximal ideal of $R$ has a maximal ideal of $\tilde{R}$ lying over it and every maximal ideal of $\tilde{R}$ contracts to a maximal ideal of $R$. Therefore $J(R) \subseteq J(\tilde{R})$. Since $\Gamma$ is module-finite over $\tilde{R}$, we have $J(\tilde{R}) \subseteq J(\Gamma)$ [every simple $\Gamma$-module $S$ is module-finite over $\tilde{R}$, hence $J(\tilde{R}) \cdot S=0$ by Nakayama's Lemma]. Therefore statement (iii) holds.

A special case of the next result is that our assumption that $\Lambda$ has no artinian ring-direct summands carries over to $\Gamma$. 
(4.1.10) $\Gamma$ has no $\Gamma$-submodules of finite length and no $R$-submodules of finite length.

Proof. Let $U \subseteq \Gamma$ be an $R$-submodule of finite length. Since $\Gamma_{Q}=\Lambda_{Q}$ we have $c U \subseteq \Lambda$ for $c \in R-\bigcup Q$. Since $\Lambda$ is an $R$-order in $\Lambda_{Q}$ we have $U \cong c U$ as $\Lambda$-modules. Since $\Lambda$ has no nonzero $R$-submodules of finite length [see (1.2.1)], we have $U=0$ as desired.

The assertion about $\Gamma$-submodules now follows from (4.1.9)(i).

Lemma 4.2. Let $S$ be a ring, integral over $R$, such that $R \subseteq S \subseteq R_{Q}$ (and recall that we are assuming that $R$ has no nilpotent elements $\neq 0$ and no artinian ringdirect summands). Let $\mathcal{M}$ be a finite set of prime ideals of $R$, and $\mathcal{N}$ the (necessarily finite) set of prime ideals $P$ of $S$ such that $P$ lies over some prime ideal in $\mathcal{M}$. Then $(R-\cup \mathcal{M})^{-1} S=S_{\mathcal{N}}$ (i.e. both localizations invert the same set of elements of $S$ ).

Proof. Since $R$ has no nilpotent elements, $R_{Q}$ is the total quotient ring of $R$, hence of $S$. By (a minor extension of) the Krull-Akizuki theorem [N '62, (33.2)] $S$ is therefore noetherian of Krull dimension 1.

Next we prove that $\mathcal{N}$ is finite. Since the noetherian $\operatorname{ring} S$ of Krull dimension 1 has only finitely many minimal primes, it suffices to show that $\mathcal{N}$ contains only finitely many maximal ideals that are not minimal primes. Each such maximal ideal $\mathbf{n} \in \mathcal{N}$ contracts to a maximal ideal of $R$, because $\mathbf{n}$ properly contains a minimal prime $\mathbf{p}$, and $\mathbf{n}$ and $\mathbf{p}$ must contract to distinct primes of $R$ by the incomparability theorem of commutative algebra [K'70, Theorem 44]. Therefore it suffices to fix a maximal ideal $\mathbf{m} \in \mathcal{M}$ and prove that $\mathbf{m}$ is contained in only finitely many maximal ideals of $S$.

Since $R$ has no nilpotent elements, $\bigcup Q(R)$ is the set of zero-divisors of $R$. Since $\mathbf{m}$ is not a minimal prime, it therefore contains a regular element $d$ of $R$. This element $d$ is a unit in $R_{Q}$ and therefore is regular in $S$. Therefore $d$ is outside of all minimal primes of $S$, and therefore the noetherian ring $S / S d$ has dimension 0 and is therefore artinian. It follows that $d$, and therefore $\mathbf{m}$, is contained in only finitely many maximal ideals of $S$, as claimed.

Now we prove equality of the two localizations of $S$ in the statement of the lemma. As in the proof of Lemma 1.5 it suffices to show that the same prime ideals of $S$ survive in both localizations of $S$.

Suppose that a prime ideal $\mathbf{p}$ of $S$ survives in $S_{\mathcal{N}}$. Then $\mathbf{p} \subseteq \mathbf{n}$ for some $\mathbf{n} \in \mathcal{N}$. Since $\mathbf{n} \cap R \in \mathcal{M}$ we see that $\mathbf{p}$ survives in $(R-\bigcup \mathcal{M})^{-1} S$.

Conversely, suppose that $\mathbf{p}$ is a prime ideal of $S$ that survives in $(R-\cup \mathcal{M})^{-1} S$. We consider two cases. Case 1: $\mathbf{p} \cap R \in \mathcal{M}$. In this case $\mathcal{P} \in \mathcal{N}$; and therefore $\mathbf{p}$ survives in $S_{\mathcal{N}}$. Case 2. $\mathbf{p} \cap R \notin \mathcal{M}$. Since $\mathbf{p}$ survives in $(R-\bigcup \mathcal{M})^{-1} S, \mathbf{p}$ (and hence $\mathbf{p} \cap R$ ) must have empty intersection with the multiplicatively closed set $R-\bigcup \mathcal{M}$ with respect to which we are localizing. Therefore there is a prime ideal $\mathbf{m} \in \mathcal{M}$ such that $\mathbf{p} \cap R \subset \mathbf{m}$. Since $S$ is integral over $R$, the going-up theorem of commutative algebra [K' 70 , Theorem 44] yields a prime ideal $\mathbf{n}$ of $S$ that contains $\mathbf{p}$ and lies over $\mathbf{m}$. But then $\mathbf{p} \subset S-\bigcup \mathcal{N}$, and hence $\mathbf{p}$ survives in $S_{\mathcal{N}}$.

Lemma 4.3 (Normalizations Localize). For every finite set $\mathcal{M}$ of maximal ideals of $R$ the localization $\Gamma_{\mathcal{M}}$ is a normalization of the $R_{\mathcal{M}}$-algebra $\Lambda_{\mathcal{M}}\left[\right.$ in $\left.\left(\Lambda_{\mathcal{M}}\right)_{Q}\right]$. 
Note. The notation $\left(\Lambda_{\mathcal{M}}\right)_{Q}$ at the end of the lemma should really be $\left(\Lambda_{\mathcal{M}}\right)_{Q\left(R_{\mathcal{M}}\right)}$. But by a slight modification of Lemma 2.3(i) these two localizations of $\Lambda_{\mathcal{M}}$ are the same, and we therefore use the shorter notation.

Proof. By the note above and Lemma 1.5 we may assume that $R \subseteq \Lambda$. Observe that this lemma generalizes the familiar fact that classical maximal orders localize, and the slight extension of this fact in [GL '88, 2.7], but its proof uses these facts. We give the short proof of these known facts, since it takes less space than explaining the difference between two sets of notations and subtleties. Thus, suppose that $\Gamma$ is a maximal $R$-order in $\Lambda_{Q}$ and $\Gamma_{\mathcal{M}} \subseteq \Upsilon \subseteq\left(\Gamma_{\mathcal{M}}\right)_{Q}$ for some $R_{\mathcal{M}}$-order $\Upsilon$. For each maximal ideal $\mathbf{m}$ of $R$ we define a $\Lambda_{\mathbf{m}}$-submodule $X(\mathbf{m}) \subseteq\left(\Gamma_{\mathbf{m}}\right)_{Q}=\left(\Gamma_{Q}\right)_{\mathbf{m}}$ as follows. If $\mathbf{m} \in \mathcal{M}$ let $X(\mathbf{m})=\Upsilon_{\mathbf{m}}$, but if $\mathbf{m} \notin \mathcal{M}$ let $X(\mathbf{m})=\Gamma_{\mathbf{m}}$. Since $\Gamma_{\mathcal{M}} \subseteq \Upsilon \subseteq\left(\Gamma_{\mathcal{M}}\right)_{Q}$ we have $X(\mathbf{m})_{Q}=\left(\Gamma_{\mathbf{m}}\right)_{Q}$ for all $\mathbf{m}$. And since $\mathcal{M}$ is a finite set we have $X(\mathbf{m})=\Gamma_{\mathbf{m}}$ for all but finitely many $\mathbf{m}$. Therefore Corollary 2.7 to our package deal theorem for localizations of submodules yields a f.g. $\Lambda$-submodule $X \subseteq \Gamma_{Q}$ such that $X_{\mathbf{m}}=X(\mathbf{m})$ for every $\mathbf{m}$. It is easily checked, locally, that $\Gamma \subseteq X, X^{2}=X$ and $1 \in X$. Therefore $X$ is an $R$-suborder of $\Gamma_{Q}=\Lambda_{Q}$ containing $\Gamma$. Maximality of $\Gamma$ then shows that $\Gamma=X$; and localizing at $\mathcal{M}$ then shows that $\Upsilon=\Gamma_{\mathcal{M}}$, proving the lemma when $\Gamma$ is an $R$-order; that is, maximal orders localize to maximal orders.

To extend the lemma to the non-module-finite case, we use our link with integral representation theory. Let $\mathcal{N}$ be the set of maximal ideals $\mathbf{n}$ of $\tilde{R}$ such that $\mathbf{n}$ lies over some maximal ideal of $R$. By Lemma $4.2, \mathcal{N}$ is a finite set. Since maximal orders localize to maximal orders, we therefore have that $\Gamma_{\mathcal{N}}$ is a maximal $\tilde{R}_{\mathcal{N}}$-order in $\left(\Gamma_{\mathcal{N}}\right)_{Q}$. By Lemma 4.2 , localizing $\tilde{R}$ and $\Gamma$ at $\mathcal{N}$ is the same as localizing them at the multiplicatively closed subset $R-\cup \mathcal{M}$. Indicating this latter localization by the subscript $\mathcal{M}$ we have that $\Gamma_{\mathcal{M}}$ is a maximal $\tilde{R}_{\mathcal{M}}$-order in $\left(\Gamma_{\mathcal{M}}\right)_{Q}$. Since, by standard commutative algebra, $\tilde{R}_{\mathcal{M}}$ is the normalization of $R_{\mathcal{M}}$ [in its total quotient ring $\left.\left(R_{\mathcal{M}}\right)_{Q}\right]$, the proof is completed by (4.1.4).

Proposition 4.4. Suppose that $R$ is semilocal and complete in its $J(R)$-adic topology. Then any normalization $\Gamma$ of $\Lambda$ is a f.g. $R$-module, hence is a maximal $R$-order in $\Lambda_{Q}$.

Proof. Since $\Lambda$ is a f.g. $R$-module and $\Gamma_{Q}=\Lambda_{Q}, \Gamma_{Q}$ is a f.g. $R_{Q}$-module, and hence $Z\left(\Gamma_{Q}\right)$ is a finitely generated $R_{Q}$-module. Since $R$ is complete, the integral closure $\tilde{R}$ of $R$ in $Z\left(\Gamma_{Q}\right)$ is a finitely generated $R$-module, by Nagata's theorem [Mts ' 80 , Cor. 2 to Theorem $31 \mathrm{C}$ ]. Since $\Gamma$ is a f.g. $\tilde{R}$-module, by (4.1.2), the proof is complete.

\section{Splitting Orders}

In this section $R$ is semilocal and has no nilpotent elements $\neq 0, R$ and $Z(\Lambda)$ have no artinian ring direct summands, and $\Lambda$ is an $R$-order in a semisimple artinian ring as defined in (1.1.1). $\Gamma$ denotes a fixed normalization of $\Lambda$, and $\tilde{R}$ denotes the integral closure of $R \cdot 1_{\Lambda}$ in $Z\left(\Lambda_{Q}\right)$. Recall that $\tilde{R}=Z(\Gamma)$, by (4.1.5). We make frequent use of the decomposition $\Gamma=\bigoplus_{k} \Gamma_{k}$ in (4.1.3), in which each $\Gamma_{k}$ is a full matrix ring over a semilocal principal ideal domain [see (4.1.8)].

Since $R$ is semilocal, so is $\tilde{R}$ [Lemma 4.2 ], and hence $\Gamma$ has only finitely many maximal ideals [by (4.1.6)]. 
Definition 5.1. A splitting order is an $R$-order $\Omega$ such that $\Omega \subseteq \Gamma$ and $\Omega_{Q}=$ $\Gamma_{Q}\left(=\Lambda_{Q}\right)$, and such that $\Omega$ has the properties (5.1.1)-(5.1.4) below.

$$
\tilde{R} \Omega=\Gamma \text {. }
$$

(5.1.2) The ring $\Omega$ decomposes like $\Gamma$, that is, $\Omega=\bigoplus_{k} \Omega_{k}$ (2-sided decomposition), where $\Omega_{k} \subseteq \Gamma_{k}$ for each $k$ [decomposition of $\Gamma$ as in (4.1.3)] and $\Omega_{k}$ and $\Gamma_{k}$ are full matrix rings of the same size over integral domains.

(5.1.3) $\tilde{R}(A \cap \Omega)=A$ (for any maximal ideal $A$ of $\Gamma$ ) and $\tilde{R}\left(\mathbf{m}^{\prime} \cap Z(\Omega)\right)=\mathbf{m}^{\prime}$ (for any maximal ideal $\mathbf{m}^{\prime}$ of $\tilde{R}$ ); and each maximal ideal of $\Omega$ is the contraction of some maximal ideal of $\Gamma$.

(5.1.4) For every maximal ideal $A$ of $\Gamma, \Omega /(A \cap \Omega)=\Gamma / A$; that is, the natural monomorphism from the left-hand side to the right-hand side is a surjection. (In particular, $A \cap \Omega$ is a maximal ideal of $\Omega$.)

One can obtain an equivalent restatement of (5.1.4) by replacing $\Gamma, \Omega$ by $\Gamma_{k}, \Omega_{k}$ respectively, and adding "for every $k$ ".

Theorem 5.2. (i) Let $F$ be a finite subset of $\Gamma$ and $\mathcal{S}$ a finite set of torsionfree (left) $\Lambda$-modules. Then there exists a splitting order $\Omega(\Lambda \subseteq \Omega \subseteq \Gamma)$ such that $\Omega \supseteq F$ and such that $\Omega L$ is $\Omega$-projective for every $L \in \mathcal{S}$.

(ii) If $\Omega$ is a splitting order and $\Omega^{\prime}$ is an $R$-order such that $\Omega \subseteq \Omega^{\prime} \subseteq \Gamma$, then $\Omega^{\prime}$ is again a splitting order.

(iii) If $\Omega$ and $\Omega^{\prime}$ are splitting orders contained in $\Gamma$, then some splitting order $\Omega^{\prime \prime} \subseteq \Gamma$ contains both $\Omega$ and $\Omega^{\prime}$.

Proof. We may assume that $R=Z(\Lambda)$. Recall that by Shirshov's theorem (4.1.1) every f.g. $R$-subalgebra of $\Gamma$ is a f.g. $R$-module. We construct $\Omega$ by a sequence of successive enlargements of $\Lambda$, keeping $\Omega \subseteq \Gamma$ at each step. At each stage of the enlargement process, $\Omega$ will be a ring that is module-finite over $R$ and therefore an $R$-order in $\Lambda_{Q}=\Gamma_{Q}$.

Property (5.1.1). By (4.1.5) there is a finite generating set $F^{\prime}$ for the $\tilde{R}$-module $\Gamma$. Let $F^{\prime \prime}$ be a finite generating set of the $R$-module $\Lambda$, and then let $\Omega$ be the $R$-algebra generated by $F^{\prime} \cup F^{\prime \prime}$. By Shirshov's theorem, $\Omega$ is a module-finite $R$ algebra, and clearly $\Lambda \subseteq \Omega \subseteq \Gamma$. We now have property (5.1.1), and any further enlargement of $\Omega$ obviously preserves this property. enl Recall that for a ring $S$ and a positive integer $n$, we have $S \cong M_{n}(T)$ for some $\operatorname{ring} T$ if and only if $S$ contains a set of $n \times n$ matrix units; that is, a set of $n^{2}$ elements $e_{i j}(1 \leq i, j \leq n)$ such that $e_{i j} e_{j k}=e_{i k}$ for all $i, j, k, e_{i j} e_{k l}=0$ whenever $j \neq k$, and $\sum_{i} e_{i i}=1$. $T$ can then be taken to be the centralizer of the $e_{i j}$.

Property (5.1.2). Enlarge $\Omega$ so that it contains all of the (finitely many) central idempotents of $\Gamma$, and a set of matrix units (of appropriate size) of each $\Gamma_{k}$. Again, further enlargements of $\Omega$ preserve these properties.

Property (5.1.4). Let $d$ be a regular element of $J(R)$, which exists by Lemma 1.3(iii) since $R=Z(\Lambda)$ has no artinian ring direct summands. Since every maximal ideal of the integral extension $\tilde{R}$ of $R=Z(\Lambda)$ lies over a maximal ideal of $R$ [K 70 , Theorem 44], we have $d \in J(\tilde{R})$. Since $\Gamma$ is a module-finite $\tilde{R}$-algebra [by (4.1.5)], we have $J(\tilde{R}) \cdot \Gamma \subseteq J(\Gamma)$, and therefore $d \in J(\Gamma)$. By the Krull-Akizuki theorem [N '62, (33.2)] the artinian ring $\tilde{R} / \tilde{R} d$ is a f.g. $R$-module. Therefore the modulefinite $\tilde{R} / \tilde{R} d$-algebra $\Gamma / \Gamma d$ is a f.g. $R$-module. Therefore a finite enlargement of $\Omega$ makes the natural map $\Omega \rightarrow \Gamma / \Gamma d$ a surjection. This enlarged $\Omega$ satisfies (5.1.4), and further enlargements preserve this property. 
Property (5.1.3). Each of the finitely many maximal ideals $\mathbf{m}^{\prime}$ of $\tilde{R}$ has a finite, (in fact, 1-element) generating set. Including these generators in $\Omega$ assures us that $\tilde{R}\left(\mathbf{m}^{\prime} \cap Z(\Omega)\right)=\mathbf{m}^{\prime}$.

Similarly we obtain $\tilde{R}(A \cap \Omega)=A$ by including, in $\Omega$, a finite set of $\Gamma$-generators of each of the finitely many of maximal ideals $A$ of $\Gamma$.

The more difficult part of the proof of (5.1.3) is to show that every maximal ideal $B$ of $\Omega$ is the contraction of some maximal ideal $A$ of $\Gamma$. It suffices to show that $\Gamma B \Gamma \neq \Gamma$. For then some maximal ideal $A$ of $\Gamma$ contains $\Gamma B \Gamma$ and therefore $A \cap \Omega \supseteq B$, whence maximality of $B$ shows that $A \cap \Omega=B$. So suppose that $\Gamma B \Gamma=\Gamma$.

Recall that $\Gamma$ is a centralizing extension of $\Omega$ [property (5.1.1)]. In [MR '87, Theorem 10.2.9] a lying-over theorem is proved for finite centralizing extensions, when the rings involved are left noetherian. However, our $\Gamma$ need not be a f.g. $\Omega$-module. Before reducing to the finite case, we recall the relevant definitions. A ring $T$ is a finite centralizing extension of a subring $S$ if there exist finitely many elements $x_{i} \in T$ such that $T=\sum_{i} S x_{i}$ and $x_{i} s=s x_{i}$ for every $s \in S$.

First note that since $\tilde{R} \Omega=\Gamma$ our assumption $\Gamma B \Gamma=\Gamma$ can be rewritten $\tilde{R} B=\Gamma$. This yields an expression of the form $\sum_{i} x_{i} b_{i}=1$ with each $x_{i} \in \tilde{R}$ and $b_{i} \in B$. Let $\Omega^{\prime}$ be the $R$-subalgebra of $\Gamma$ generated by $\Omega$ and the $x_{i}$. By Shirshov's theorem $\Omega^{\prime}$ is a f.g. $R$-module. Also, $\Omega^{\prime} B \Omega^{\prime}=\Omega^{\prime}$. Since $\Omega^{\prime}$ is a finite centralizing extension of $\Omega$, the lying-over theorem for finite centralizing extensions yields a prime ideal $B^{\prime}$ of $\Omega^{\prime}$ such that $B^{\prime} \cap \Omega=B$, and this contradicts the relation $\Omega^{\prime} B \Omega^{\prime}=\Omega^{\prime}$, completing our proof of (5.1.3).

This completes the proof of parts (i) and (ii), except for the statement that we can choose $\Omega$ large enough to make each $\Omega L \Omega$-projective. This is straightforward, and is done in [GL '89, 2.8]. Part (iii) is an immediate consequence of Shirshov's theorem.

Lemma 5.3. Let $\Omega$ be a splitting order. Then

(i) The sets of maximal (2-sided) ideals of all four rings $\Gamma, \Omega, Z(\Gamma), Z(\Omega)$ are in one-to-one correspondence via contraction.

(ii) $J(\Gamma) \cap \Omega=J(\Omega)$ and $\tilde{R} \cdot[J(\Omega)]=J(\Gamma)$.

Proof. (i) The statement about $\Gamma$ and $Z(\Gamma)$ was already noted in (4.1.6). The one-to-one correspondence of maximal ideals between $\Gamma$ and $\Omega$ is part of (5.1.3) and (5.1.4). Most of this correspondence for $\tilde{R}$ and $Z(\Omega)$ was stated in (5.1.3). We need to show that every maximal ideal of $Z(\Omega)$ is the contraction of some maximal ideal of $\tilde{R}$. Since $\tilde{R}$ is integral over $Z(\Omega)$, this follows from the lyingover and incomparability theorems of commutative algebra [K '70, Theorem 44]. Finally, the relation between the maximal ideals of $\Omega$ and its center follows from the situations already considered.

(ii) The first equality follows from statement (i) as soon as we know that the Jacobson radical of each ring is the intersection of its maximal ideals. This follows from Lemma 1.6(i), applied to the $R$-algebra $\Omega$ and the $\tilde{R}$-algebra $\Gamma$.

We define the symmetric product $\mathcal{P}\left(D_{1}, \ldots, D_{n}\right)$ of ideals $D_{1}, \ldots, D_{n}$ of a ring $S$. This consists of the sum of all $n$ ! possible terms, where each term is the product of all $n$ of the ideals $D_{k}$, taken in some order without repetition. Thus $\mathcal{P}\left(D_{1}, D_{2}\right)=$ $D_{1} D_{2}+D_{2} D_{1}$. We claim that, if $S=D_{i}+D_{j}$ whenever $i \neq j$, then: 


$$
\bigcap_{i} D_{i}=\mathcal{P}\left(D_{1}, \ldots, D_{n}\right)
$$

We have $S=\left(D_{1}+D_{2}\right) \cdots\left(D_{1}+D_{n}\right)=D_{1}+\left(D_{2} \cdots D_{n}\right)$. Left-multiplying this by $I=\bigcap_{i} D_{i}$ yields $I=I D_{1}+I\left(D_{2} \cdots D_{n}\right)$. Since $I \subseteq \bigcap_{i>2} D_{i}$, induction shows that $I D_{1} \subseteq \mathcal{P}\left(D_{1}, \ldots, D_{n}\right)$. The term $I\left(D_{2} \cdots D_{n}\right)$ in our expression for $I$ is obviously contained in $\mathcal{P}\left(D_{1}, \ldots, D_{n}\right)$, and this proves the inclusion $(\subseteq)$ in (5.3.1). The opposite inclusion is obvious.

To prove the second equality in statement (ii), let $A_{1}, \ldots, A_{n}$ be the maximal ideals of $\Gamma$, and let $D_{i}=A_{i} \cap \Omega$. Then, by statement (i) the distinct maximal ideals of $\Omega$ are $D_{1}, \ldots, D_{n}$; and $\tilde{R} D_{i}=A_{i}$ by (5.1.3). We have $J(\Omega)=\bigcap_{i} D_{i}$. Therefore (5.3.1) yields

$$
\tilde{R} \cdot J(\Omega)=\tilde{R}\left(\bigcap_{i} D_{i}\right)=\tilde{R} \mathcal{P}\left(D_{1}, \ldots, D_{n}\right)=\mathcal{P}\left(A_{1}, \ldots, A_{n}\right)=J(\Gamma),
$$

completing the proof.

Lemma 5.4. Let $\Omega$ be a splitting order, and let $S$ be a simple (left) $\Gamma$-module. Then:

(i) $S$ is a simple $\Omega$-module, and all simple $\Omega$-modules are of this form.

(ii) Nonisomorphic simple $\Gamma$-modules remain non-isomorphic as $\Omega$-modules.

(iii) $\Gamma \otimes_{\Omega} S \cong S$ as $\Gamma$-modules.

Proof. The annihilator $A$ of $S$ in $\Gamma$ is a maximal ideal of $\Gamma$, by Lemma 1.6 applied to $\Gamma$ as an $\tilde{R}$-order. Since $\Gamma / A$ is a simple artinian ring, we have $\Gamma / A \cap S^{m}$ as $\Gamma$-modules, for some $m$. By (5.1.4) we have $\Omega /(A \cap \Omega) \cong S^{m}$ as $\Omega$-modules, and $S$ is a simple $\Omega$-module. Also, $\Gamma(A \cap \Omega)=A$ by (5.1.3) and (5.1.1). Therefore

$$
\left(\Gamma \otimes_{\Omega} S\right)^{m} \cong \Gamma \otimes_{\Omega} S^{m} \cong \Gamma \otimes_{\Omega} \Omega /(A \cap \Omega) \cap \Gamma / \Gamma(A \cap \Omega)=\Gamma / A \cong S^{m}
$$

Since $S$ is a simple $\Gamma$-module, this implies statement (iii).

To complete the proof of (i), let $T$ be a simple $\Omega$-module. We show that $T \cong S$ for some $S$ as above. By Lemma 1.6, the annihilator $D$ of $S$ in $\Omega$ is a maximal ideal of $\Omega$. Therefore $T$ is the unique simple module over the simple artinian ring $\Omega / D$. By Lemma 5.3 we have $D=A \cap \Omega$ for some maximal ideal $A$ of $\Gamma$. It follows that $T$ is also the unique simple $\Gamma / A$-module, hence a simple $\Gamma$-module.

To prove (ii), note that the annihilators of non-isomorphic simple $\Gamma$-modules $S, S^{\prime}$ are distinct maximal ideals of $\Gamma$, say $A, A^{\prime}$ respectively. The annihilators of $S$ and $S^{\prime}$ in $\Omega$ are $A \cap \Omega$ and $A^{\prime} \cap \Omega$, respectively, and these are distinct maximal ideals of $\Omega$ by Lemma 5.3. Therefore $S$ and $S^{\prime}$ are not isomorphic $\Omega$-modules.

Now we obtain the second main result of this section: the structure of projective $\Omega$-modules is the same as the structure of projective $\Gamma$-modules (equivalently, the same as the structure of projective $\Omega_{Q}$-modules).

Theorem 5.5. Let $\Omega$ be a splitting order, and let $P, P^{\prime}$ be f.g. projective $\Omega$-modules. Then:

(i) $P$ decomposes like $P_{Q}$; that is, $P=\bigoplus_{i} P_{i}$ where each $P_{i}$ is indecomposable, each $\left(P_{i}\right)_{Q}$ is an indecomposable (hence simple) $\Omega_{Q}$-module, and $P_{i} \cong P_{j}$ if and only if $\left(P_{i}\right)_{Q} \cong\left(P_{j}\right)_{Q}$. 
(ii) $P \cong P^{\prime} \Longleftrightarrow P_{Q} \cong P_{Q}^{\prime}$. Moreover, every f.g. (necessarily projective) $\Omega_{Q^{-}}$ module is isomorphic to $P_{Q}$ for some f.g. projective $\Omega$-module $P$.

(iii) The Krull-Schmidt theorem holds for projective $\Omega$-modules.

Proof. By (5.1.2) we may suppose that $\Omega=\Omega_{1}$ and $\Gamma=\Gamma_{1}$; that is, $\Omega$ and $\Gamma$ are $R$-orders in the simple artinian ring $\Omega_{Q}=\Gamma_{Q}$. Then $\Omega$ and $\Gamma$ are full $n \times n$ matrix rings over integral domains. Let $L=\Omega e_{11}$ where $e_{11}$ is the $n \times n$ matrix with 1 in its $(1,1)$-position and 0 elsewhere. If we can show that

$$
P \cong L^{s} \text { for some unique integer } s \geq 0,
$$

then all three assertions of the theorem follow immediately. Uniqueness of $s$ holds because $L$ is a noetherian module.

To show the existence of $s$, first note that $\Gamma \otimes_{\Omega} L \cong \Gamma L=\Gamma e_{11}$. Since $\Gamma \otimes_{\Omega} P$ is $\Gamma$-projective and $\Gamma$ is an $n \times n$ matrix ring over a principal ideal domain, $\Gamma e_{11}$ is the unique indecomposable projective $\Gamma$-module, and we therefore have $\Gamma \otimes P \cong(\Gamma \otimes L)^{s}$ for some $s$. To complete the proof we cancel $\Gamma$ from this isomorphism. Reducing both sides modulo $J(\Gamma)=\tilde{R} J(\Omega)$ [Lemma 5.3] and using right-exactness of $\otimes$ yields

$$
\Gamma \otimes_{\Omega} P /(J(\Omega) \cdot P) \cong \Gamma \otimes_{\Omega}(L / J(\Omega) \cdot L)^{s}
$$

Since $P /(J(\Omega) \cdot P)$ and $(L / J(\Omega) \cdot L)^{s}$ are semisimple $\Omega$-modules, Lemma 5.4 allows us to cancel $\Gamma$ from the isomorphism in (5.5.2). Therefore $P /(J(\Omega) \cdot P) \cong(L / J(\Omega) \cdot L)^{s}$ as $\Omega$-modules. Uniqueness of the projective cover (see, e.g. [LO, 8.8]) then yields $P \cong L^{s}$.

Corollary 5.6. Each prime ring-direct summand $\Omega_{k}$ of $\Omega$ has, up to isomorphism, exactly one indecomposable f.g. projective module-which we call $B_{k}$ from now on.

Proof. Since $\left(\Omega_{k}\right)_{Q}$ is a simple artinian ring, the result follows immediately from the theorem.

\section{Completions of Orders, Artinian Pairs}

Throughout this section $R$ is semilocal and has no nilpotent elements $\neq 0$, and $\Lambda$ is an $R$-order in the semisimple artinian ring $\Lambda_{Q}$, as in (1.1.1). We also make the nontriviality assumption that $\Lambda$ [equivalently, $Z(\Lambda)$ ] has no artinian ring-direct summands. By (4.1.10), $\Gamma$ and $Z(\Gamma)$ likewise have no artinian ring-direct summands.

According to the "abstract" Package Deal Theorem for completions 3.4, a f.g. $\hat{\Lambda}$-module $X$ is the completion of some f.g. $\Lambda$-module if and only if $X_{Q}$ is the completion of some $\Lambda_{Q}$-module. In order to make use of this, we need an easily verifiable criterion for the latter condition to be fulfilled. Let $\Gamma$ denote a normalization of $\Lambda$. The condition is that there exists a f.g. projective module $P$ over $\Gamma$ [alternatively, over some conveniently chosen splitting order $\Omega]$ such that $\hat{R} \otimes_{R} P_{Q} \cong X_{Q}$. The Package Deal Theorem for completions of orders, in this section, proves this and reduces its verification, for a specific $X$, to the comparison of two arrays of nonnegative integers, called "ranks" (which describe the decomposition of $X_{Q}$ ) and "splitting numbers" (which describe how the decomposition of $\Gamma$ is refined by passing to $\ddot{\Gamma}=\hat{R} \otimes_{R} \Gamma$ ).

Caution. As mentioned in the discussion of $\S 6$ in the Introduction to this paper, the notation $\ddot{\Gamma}$ is chosen to emphasize that the ring $\ddot{\Gamma}=\hat{R} \otimes_{R} \Gamma$ is not an "adic" 
completion of $\Gamma$ when $\Gamma$ is not module-finite over $R$. Nor is it noetherian in this situation.

We prepare for the proof of our Package Deal Theorem for completions of orders by giving detailed descriptions of the projective modules over the rings $\ddot{\Gamma}, \hat{\Omega}(\Omega$ any splitting $R$-order contained in $\Gamma$ ), and their localizations $\ddot{\Gamma}_{Q}=\hat{\Omega}_{Q}$. An unexpected technical difficulty is the proof that indecomposable projective $\Omega_{Q^{-}}$and $\Gamma_{Q^{-}}$-modules remain indecomposable after tensoring with $\hat{R}$ (equivalently, primitive idempotents of these rings remain primitive in $\hat{\Omega}_{Q}=\ddot{\Gamma}_{Q}$ ).

When dealing only with torsionfree $\Lambda$-modules, there is an alternative to working with $\hat{\Lambda}$. One can work with a simpler structure involving modules over an associated pair of artinian rings. In the last part of this section we obtain the package deal theorem associated with artinian pairs.

Notation 6.1. The results and proofs of this section require a great deal of technical notation, much of which we collect here. Subscript $Q-$ as in $\Lambda_{Q}$ or $M_{Q}$ always denotes the $Q(R)$-localization, that is, the localization formed by using the denominator set $R-\bigcup Q(R)$. In particular, the notation $\hat{\Lambda}_{Q}$ denotes the localization formed by using the denominator set $R-\bigcup Q(R)$ rather than its counterpart $\hat{R}-\bigcup Q(\hat{R})$ for $\hat{R}$. Recall that the ring $\hat{\Lambda}_{Q}=\hat{R} \otimes_{R} R_{Q} \otimes_{R} \Lambda$ is artinian, by (3.1.2).

Fix a normalization $\Gamma$ of $\Lambda$ for the rest of this section. The notation $\Omega$ always denotes a splitting $R$-order contained in $\Gamma$ (but not always the same splitting order). By (5.1.2) we have 2-sided decompositions:

$$
\Omega=\bigoplus_{k} \Omega_{k} \quad \text { and } \quad \Gamma=\bigoplus_{k} \Gamma_{k}
$$

where each $\Omega_{k} \subseteq \Gamma_{k}$ and $\left(\Omega_{k}\right)_{Q}=\left(\Gamma_{k}\right)_{Q}$, a simple artinian ring.

Simple modules $S_{k i}$ and maximal ideals $M_{k i}^{\prime}$. The four main rings dealt with in this section $-\Gamma, \ddot{\Gamma}, \Omega, \hat{\Omega}$ - all turn out to have the same finite family of simple (left) modules. For our formal definition, we begin with $\Gamma$. For each index $k$ in (6.1.1) let $S_{k 1}, S_{k 2}, \ldots$ be a set of representatives of the isomorphism classes of simple (left) $\Gamma_{k}$-modules. Then the set $\left\{S_{k i}\right\}$ (where both $k$ and $i$ are allowed to vary) is a set of representatives of the isomorphism classes of simple $\Gamma$-modules. Since $R$ is semilocal in this section, $\left\{S_{k i}\right\}$ is a finite set as we observe in the next paragraph.

Since $\Gamma$ is a module-finite $\tilde{R}$-algebra [see (4.1.2)], its set of isomorphism classes of simple left modules $S$ corresponds bijectively with its set of maximal ideals via $S \rightarrow \operatorname{ann}_{\Gamma} S$ [Lemma 1.6]. Moreover, since $R$ is semilocal, $\Gamma$ has only finitely many maximal ideals, as explained immediately before Definition 5.1. Therefore each $\Gamma_{k}$ has only finitely many simple left modules $S_{k i}$, as mentioned above. We index the maximal ideals of each $\Gamma_{k}$ with the same set of ordered pairs $(k, i)$ used for simple modules by setting $M_{k i}^{\prime}=\operatorname{ann}_{\Gamma_{k}} S_{k i}$.

Splitting numbers $s_{k i}$. Each $\Gamma_{k}$ is a full matrix ring over a principal ideal domain, by (4.1.8), and therefore has a unique (up to isomorphism) indecomposable f.g. projective left module $\Gamma_{k} e_{k}$, where $e_{k}$ is an arbitrary primitive idempotent of $\Gamma_{k}$. Since the ring $\Gamma_{k} / M_{k i}^{\prime}$ is simple artinian, its module $\Gamma_{k} e_{k} / M_{k i}^{\prime} e_{k}$ is the direct sum of some number $s_{k i}$ of copies of the simple $\Gamma_{k}$-module annihilated by $M_{k i}^{\prime}$. That is,

$$
\Gamma_{k} e_{k} / M_{k i}^{\prime} e_{k} \cong\left(S_{k i}\right)^{s_{k i}} \quad \text { where } M_{k i}^{\prime}=\operatorname{ann}_{\Gamma_{k}} S_{k i} \text {. }
$$

We call $s_{k i}$ the splitting number of $M_{k i}^{\prime}$, or the $(k, i)$-splitting number of $\Gamma$. We often write $\bar{\Gamma}=\Gamma / J(\Gamma)$ and $\bar{\Gamma}_{k}=\Gamma_{k} / J\left(\Gamma_{k}\right)$. Since $J\left(\Gamma_{k}\right)$ is the intersection of the 
maximal ideals of $\Gamma_{k}$, we can rewrite (6.1.2) in the form

$$
\bar{\Gamma}_{k} \bar{e}_{k} \cong \bigoplus_{i}\left(S_{k i}\right)^{s_{k i}} \text {. }
$$

The simple $\Gamma$-modules coincide with the simple $\Omega$-modules (for every $\Omega$ ). This follows from the relation

$$
\Omega / J(\Omega)=\Gamma / J(\Gamma)=\bar{\Gamma}
$$

where the first equality means that the natural map is a bijection. Relation (6.1.4) follows imediately from (5.1.4) and Lemma 5.3.

Caution. When dealing with artinian pairs, at the end of this section, we use the overbar notation $\bar{\Omega}$ to denote reduction modulo a conductor ideal. Whenever we use the overbar notation we will include reminders of what is intended.

Maximal ideals $M_{k i}$. By Corollary 5.6, $\Omega_{k}$ has a unique indecomposable f.g. projective left module that we call $B_{k}$; and by Lemma 5.3 the maximal ideals of $\Omega_{k}$ are the ideals $M_{k i}=M_{k i}^{\prime} \cap \Omega_{k}$, with distinct subscripts $(k, i)$ corresponding to distinct ideals $M_{k i}$. The following facts refine (6.1.4):

$$
B_{k} / M_{k i} B_{k} \cong\left(S_{k i}\right)^{s_{k i}} \quad \text { where } M_{k i}=\operatorname{ann}_{\Omega_{k}} S_{k i}=M_{k i}^{\prime} \cap \Omega_{k},
$$

and therefore we also refer to $s_{k i}$ as the splitting number of $M_{k i}$.

To prove (6.1.5), note first that the natural monomorphism $\Omega_{k i} / M_{k i} \rightarrow \Gamma_{k} / M_{k i}^{\prime}$ is a bijection by (5.1.4). Therefore the simple $\Gamma_{k}$-modules coincide with the simple $\Omega_{k}$-modules, as claimed. By (5.1.2), $\Omega_{k}$ and $\Gamma_{k}$ are full matrix rings of the same size $n_{k} \times n_{k}$ over integral domains. It follows that $\left(B_{k} / M_{k i} B_{k}\right)^{n_{k}} \cong \Omega_{k} / M_{k i}$ and $\left(\Gamma_{k} e_{k} / M_{k i}^{\prime}\right)^{n_{k}} \cong \Gamma_{k} / M_{k i}^{\prime}$. The natural isomorphism $\Omega_{k i} / M_{k i} \cong \Gamma_{k} / M_{k i}^{\prime}$, together with (6.1.2), now yields (6.1.5).

Matrix size. Let $\Delta$ be an integral domain (perhaps a division ring). We define the matrix size of any ring isomorphic to the full $n \times n$ matrix ring $M_{n}(\Delta)$ to be the integer $n$. In particular, the matrix sizes of the rings $\Gamma_{k}$ and $\Omega_{k}$ will often be denoted by $n_{k}$, as in the preceding paragraph.

The foregoing definitions of splitting numbers were stated in terms of left modules. We claim that using right modules would yield the same splitting numbers. This is an immediate consequence of the following easily derived formula:

$$
s_{k i}=\frac{\text { matrix size of } \Gamma_{k} / M_{k i}^{\prime}}{\text { matrix size of } \Gamma_{k}}
$$

Finally, we note that the set of splitting numbers $s_{k i}$ is independent of which normalization $\Gamma$ we choose because, since $R$ is semilocal, $\Gamma$ is unique up to conjugacy, by (4.1.7).

Rings $\hat{\Gamma}, \ddot{\Gamma}$. The notation $\hat{\Gamma}$ denotes the completion of $\Gamma$ with respect to $J(\tilde{R})$, where, as in (4.1.2), $\tilde{R}=Z(\Gamma)$. Since, in our semilocal situation, $\tilde{R}$ is a direct sum of principal ideal domains and $\Gamma$ is a direct sum of classical maximal orders over the ring-direct summands of $\tilde{R}$ in simple algebras [see (4.1.3)], $\hat{\Gamma}$ is a direct sum of classical maximal orders over discrete valuation rings in simple algebras, and we can make the identification $\hat{\Gamma}=(\tilde{R})^{\wedge} \otimes_{\tilde{R}} \Gamma$ as $(\tilde{R})^{\wedge}$-algebras.

$\hat{\Gamma}$ is also the $J(R)$-adic completion of $\Gamma$, since $J(R) \cdot 1_{\Gamma} \subseteq J(\tilde{R})$ and $J(\tilde{R})$ is nilpotent modulo $J(R) \cdot \tilde{R}$ (see the proof of Lemma 6.2). But this fact does not seem particularly useful in the situations that interest us, because when $\Gamma$ is not module-finite over $R$, the rings $\hat{\Gamma}$ and $\ddot{\Gamma}=\hat{R} \otimes_{R} \Gamma$ are never isomorphic. This 
follows, for example, from the fact that the ring $\ddot{\Gamma}$ is not noetherian in this situation [Lemma 6.7]. Furthermore, it has nilpotent ideals since $\hat{R}$ does.

The ring $\ddot{\Gamma}=\hat{R} \otimes_{R} \Gamma$, despite being non-noetherian, has a number of finiteness conditions that we need [see Lemma 6.7 below], and plays a more central role in this section than $\hat{\Gamma}$ does because it is more directly related to the flatness properties of $\hat{R}$ as an $R$-module.

We shall use the following simple fact often.

(6.1.7) For every $R$-module $U$ of finite length we have $U=\hat{R} \otimes_{R} U$ (that is, the natural map from the left-hand side to the right-hand side is a bijection). In particular, $U$ is an $\hat{R}$-module. Moreover, every $R$-submodule of $U$ is an $\hat{R}$-submodule.

Proof. Since $U$ is f.g. as an $R$-module, $\hat{R} \otimes_{R} U$ is a $J(R)$-adic completion of $U$; and since $U$ has finite length, this can be identified with $U$ itself, proving the first assertion. To prove the last assertion, let $V$ be an $R$-submodule of $U$. Then, by the first assertion, $\hat{R} \otimes_{R} V=V$; and therefore $V$ is an $\hat{R}$-module. Moreover, since $\hat{R}$ is a flat $R$-module, the inclusion $V \subseteq U$ induces an inclusion $\hat{R} \otimes V \hookrightarrow \hat{R} \otimes_{R} U$, showing that $V$ is an $\hat{R}$-submodule of $U$.

Lemma 6.2. The $J(R)$-adic completion of $\Lambda$ equals the $J(Z(\Lambda)$ )-adic completion of $\Lambda$.

Proof. Let $S=Z(\Lambda)$. It suffices to show that the ideals $J(R) \cdot S$ and $J(S)$ generate the same adic topology on $S$. To prove this it suffices to show that each of these ideals contains a power of the other. Since $S$ is a module-finite $R$-algebra, $J(R)$ annihilates every simple $S$-module (as in the proof of Lemma 1.6), and hence $J(R) \cdot S \subseteq J(S)$. On the other hand, $S /(J(R) \cdot S)$ is a module-finite algebra over the artinian ring $R / J(R)$, and its radical is therefore nilpotent. Therefore some power of $J(S)$ is contained in $J(R) \cdot S$.

Lemma 6.3. $Z(\hat{\Lambda})=Z(\Lambda)^{\wedge}$.

Proof. We sketch a proof of this known result because we do not know a reference to it. Let $a_{1}, a_{2}, \ldots$ be a finite set of generators of $\Lambda$ as an $R$-module. Then they also generate $\hat{\Lambda}$ as an $\hat{R}$-module. Let $A_{i}=\left\{x a_{i}-a_{i} x \mid x \in \Lambda\right\}$. Then let

$$
f: \Lambda \rightarrow \bigoplus_{i} A_{i}
$$

be the $R$-homomorphism whose $i^{\text {th }}$ coordinate map is $x \rightarrow x a_{i}-a_{i} x$. The kernel of this map is $Z(\Lambda)$; and since the $a_{i}$ generate $\hat{\Lambda}$ the kernel of the induced map $\hat{f}: \hat{\Lambda} \rightarrow \bigoplus_{i} \hat{A}_{i}$ is $Z(\hat{\Lambda})$. Since $J(R)$-adic completion is accomplished by tensoring with the flat $R$-module $\hat{R}$, the kernel of $\hat{f}=1 \otimes f$ equals $(\operatorname{ker} f)^{\wedge}$, as claimed.

Corollary 6.4. $\hat{\Lambda}$ is an indecomposable ring if and only if $Z(\Lambda)$ is a local ring.

Proof. By Lemma 6.2 we may assume that $R=Z(\Lambda)$. Ring-direct-sum decompositions of $\hat{\Lambda}$ arise from writing its identity element as a sum of orthogonal, central idempotents. Therefore $\hat{\Lambda}$ is an indecomposable ring if and only if $Z(\hat{\Lambda})$ is. Since $Z(\hat{\Lambda})=Z(\Lambda)^{\wedge}$ [Lemma 6.3], our corollary is reduced to a problem in commutative rings. The proof is completed by the fact that if $R=Z(\Lambda)$ is semilocal with, say, $m$ maximal ideals, then $\hat{R}$ is the direct sum of $m$ local rings. [B '72, Chap. III, $\S 2.13$, final Cor.]. 
As in [LO], we call a ring $T$ matrix-local if $T / J(T)$ is a simple artinian ring.

Lemma 6.5. Let $\mathbf{m}_{k i}=M_{k i} \cap Z\left(\Omega_{k}\right)$. Then we have the ring-direct sum

$$
\hat{\Omega}=\bigoplus_{k i}\left(\Omega_{k}\right)^{\wedge(k i)}
$$

where $\left(\Omega_{k}\right)^{\wedge(k i)}$ denotes the $\mathbf{m}_{k i}$-adic completion of (the $\mathbf{m}_{k i}$-localization of) $\Omega_{k}$, and is a matrix-local, hence indecomposable ring with $J\left(\left(\Omega_{k}\right)^{\wedge(k i)}\right)=M_{k i}{ }^{\wedge(k i)}$.

Proof. By Lemma 6.2 we may assume that $R=Z(\Omega)$. Then $R=\bigoplus_{k} R_{k}$ where $R_{k}=Z\left(\Omega_{k}\right)$. Since the sets of maximal ideals of $\Omega$ and its center are in oneto-one correspondence via contraction [Lemma 5.3], $\left\{\mathbf{m}_{k i}\right\}$ is the set of maximal ideals of $R_{k}$. As with all semilocal commutative rings we therefore have $\hat{R}_{k}=$ $\bigoplus_{i} R_{k}{ }^{\wedge(k i)}$ [B '72, Chap. III, §2.13, final Cor.], and this yields decomposition (6.5.1). So it only remains to prove that each term $\left(\Omega_{k}\right)^{\wedge(k i)}$ is matrix-local with the indicated Jacobson radical. After localizaing at $\mathbf{m}_{k i}$ and changing notation, $R=Z(\Omega)$ becomes a local ring and $\Omega$ has only one maximal ideal, namely $J(\Omega)$, and $\Omega$ is matrix-local. What we want to prove is that $J(\hat{\Omega})=J(\Omega)^{\wedge}$ and the ring $\hat{\Omega} / J(\hat{\Omega})$ is simple artinian. This follows from Proposition 3.8.

Theorem 6.6 (Projective $\hat{\Omega}$-modules). $\quad$ (i) For each $(k, i)$ there is a unique indecomposable f.g. projective (left) $\hat{\Omega}_{k}$-module $U_{k i}$ that maps $\hat{\Omega}$-linearly onto $S_{k i}$. Every indecomposable f.g. projective $\hat{\Omega}$-module is isomorphic to some $U_{k i}$.

(ii) We have $\hat{B}_{k} \cong \bigoplus_{i}\left(U_{k i}\right)^{s_{k i}}$, where $B_{k}$ denotes the unique indecomposable f.g. projective $\Omega_{k}$-module; and $\hat{\Omega}_{k} \cong \bigoplus_{i}\left(U_{k i}\right)^{n_{k} s_{k i}}$, where $n_{k}$ is the matrix size of $\Gamma_{k}$ (equivalently, of $\Omega_{k}$ ).

Proof. By Lemma 6.2 we may assume that $R=Z(\Omega)$. We have $B_{k} / M_{k i} B_{k} \cong$ $\left(S_{k i}\right)^{s_{k i}}$ by (6.1.5). Since $\Omega$-modules of finite length have finite $R$-length [Lemma 3.7], they equal their completions, and therefore tensoring with $R^{\wedge(k i)}$ yields

$$
\left(B_{k}\right)^{\wedge(k i)} /\left(M_{k i}\right)^{\wedge(k i)} B_{k}^{\wedge(k i)} \cong\left(S_{k i}\right)^{s_{k i}} .
$$

Since the complete ring $\Omega^{\wedge(k i)}$ is semiperfect, its simple module $S_{k i}$ has a projective cover $U_{k i} \rightarrow S_{k i}$, and this yields a projective cover $\left(U_{k i}\right)^{s_{k i}} \rightarrow\left(S_{k i}\right)^{s_{k i}}$. Since $\left(M_{k i}\right)^{\wedge(k i)}$ is the unique maximal ideal of $\Omega^{\wedge(k i)}$ [Lemma 6.5], the natural homomorphism $\left(B_{k}\right)^{\wedge(k i)} \rightarrow\left(S_{k i}\right)^{s_{k i}}$ given by (6.6.1) is also a projective cover. Therefore uniqueness of the projective cover (see, e.g., [LO, 8.8]) shows that $\left(B_{k}\right)^{\wedge(k i)} \cong$ $\left(U_{k i}\right)^{s_{k i}}$. The rest of the proof is straightforward.

We use the notation $N(T)$ for the sum of all nilpotent ideals of a ring $T$. For all the rings we consider, this turns out to be the nilradical of $T$, in the sense that $N(T)$ is the largest nilpotent ideal of $T$ and $T / N(T)$ has no nilpotent ideals.

Lemma 6.7. (i) The ring $\ddot{\Gamma}$ is neither left nor right noetherian if $\Gamma$ is not a f.g. $R$-module.

(ii) $N(\ddot{\Gamma})$ is a nilpotent ideal, and $J(\ddot{\Gamma})=\ddot{\Gamma} \delta$ where $\delta$ is any element of $\Gamma$ such that $J(\Gamma)=\Gamma \delta$.

(iii) $\ddot{\Gamma} / N(\ddot{\Gamma})$ is a noetherian ring. In fact, it is a maximal $R^{\prime}=\hat{R} / N(\hat{R})$-order in the semisimple artinian ring $(\ddot{\Gamma} / N(\ddot{\Gamma}))_{Q\left(R^{\prime}\right)}=(\ddot{\Gamma} / N(\ddot{\Gamma}))_{Q(R)}$. 
Proof. We may suppose that $R \subseteq \Lambda$. We first establish some facts that will be needed again in the proof of Theorem 6.12. First of all,

$$
J(R) \subseteq J(\ddot{\Gamma}) .
$$

It suffices to show that, for all $c \in J(R)$ and all $x \in \ddot{\Gamma}$, the element $1-x c$ is a unit of $\ddot{\Gamma}$. After writing $x=\sum \hat{r}_{i} \gamma_{i}\left(\hat{r}_{i} \in \hat{R}, \gamma_{i} \in \Gamma\right)$ we see that there is a splitting $R$-order $\Omega \subseteq \Gamma$ such that $x \in \hat{\Omega}$ [Theorem 5.2]. We have $J(R) \subseteq J(\Omega)$ since $\Omega$ is module-finite over $R$; and $J(\Omega) \subseteq J(\hat{\Omega})$ by Proposition 3.8. Therefore $1-x c$ is invertible in the subring $\hat{\Omega}=\hat{R} \otimes_{R} \Omega$ of $\ddot{\Gamma}$ and hence in $\ddot{\Gamma}$ itself, proving (6.7.1).

Next we show:

$$
J(\ddot{\Gamma})=\hat{R} J(\Gamma) .
$$

Throughout this section we are assuming that $Z(\Lambda)$ has no artinian ring-direct summands. Therefore $J(R)$ contains a regular element $c$ of $R$ [Lemma 1.4(iii)]. Since $c$ becomes a unit in $R_{Q}$, hence in $\Gamma_{Q}$, it remains regular in $\Gamma$. Moreover, by (4.1.9), $c \in J(\Gamma)$.

Since $c$ is a regular element of $R, \Gamma / \Gamma c$ has finite length as an $R$-module, by (4.1.9). Therefore $\Gamma / \Gamma c=\ddot{\Gamma} / \ddot{\Gamma} c$, and every $R$-submodule of the right-hand side is an $\hat{R}$-submodule, by (6.1.7). Since $c \in J(\Gamma)$, the radical of the left-hand side, considered as a $\Gamma$-module, is $J(\Gamma) / \Gamma c$. Therefore the radical of the right-hand side, considered as a $\Gamma$-module, is $X=[J(\Gamma)+\ddot{\Gamma} c] / \ddot{\Gamma} c$. Since every $R$-submodule of the right-hand side is an $\hat{R}$-module, $X$ equals the radical of $\ddot{\Gamma} / \ddot{\Gamma} c$ as an $\hat{R} \Gamma=\ddot{\Gamma}$ module, and $X=[\hat{R} J(\Gamma)+\ddot{\Gamma} c] / \ddot{\Gamma} c$. It follows that $J(\ddot{\Gamma})=\hat{R} J(\Gamma)+\ddot{\Gamma} c$. To complete the proof of the claim it therefore suffices to show that $\ddot{\Gamma} c \subseteq \hat{R} J(\Gamma)$. Since $\ddot{\Gamma} c=\hat{R} \Gamma c$ it suffices to show that $\Gamma c \subseteq J(\Gamma)$. Since $\Lambda$ is module-finite over $R$ we have $c \in J(R) \subseteq J(Z(\Lambda))$, and $J(Z(\Lambda)) \subseteq J(\Gamma)$ by (4.1.9). This completes the proof of (6.7.2).

Let $\bar{\Gamma}=\Gamma / J(\Gamma)$. We claim that $\bar{\Gamma}$ has finite length as an $R$-module and

$$
\ddot{\Gamma} / J(\ddot{\Gamma})=\bar{\Gamma} .
$$

Choose $c$ as in the proof of (6.7.2). Then $\Gamma / \Gamma c$ has finite length as an $R$-module, as already observed. Moreover, $\Gamma c \subseteq J(\Gamma)$, as shown near the end of the proof of (6.7.2). Therefore the $R$-algebra $\Gamma / \Gamma c$ can be mapped onto $\bar{\Gamma}$, and therefore $\bar{\Gamma}$ has finite length as claimed. Because of this finite length, (6.1.7) shows that $\bar{\Gamma}=\hat{R} \otimes_{R} \bar{\Gamma}=\ddot{\Gamma} / \hat{R} J(\Gamma)$, and (6.7.2) completes the proof of (6.7.3).

Statement (ii), the assertion about $J(\ddot{\Gamma})$. This follows immediately from (6.7.2) together with the facts that $\ddot{\Gamma}=\hat{R} \Gamma$ and $J(\Gamma)=\Gamma \delta$ [see (4.1.8)].

(i). Since $\Gamma$ is not a f.g. $R$-module but is a f.g. $\tilde{R}$-module, the ring $\tilde{R}$ is not a f.g. $R$-module. We first prove the commutative case of statement (i), that is, $\ddot{R}=\hat{R} \otimes_{R} \tilde{R}$ is not a noetherian ring. Let $J(\tilde{R})=\tilde{R} \pi$.

Claim. $\pi$ is a regular element of $\ddot{R}$; that is, the natural image $1 \otimes \pi$ of $\pi$ in $\ddot{R}$ is regular. Our assumption that $Z(\Lambda)$ has no artinian ring-direct summands, implies that $Z(\Gamma)$ has no artinian ring-direct summands, by (4.1.10). Therefore $J(\tilde{R})$ contains regular elements of $\tilde{R}$ [Lemma 1.3], and therefore its generator $\pi$ is regular. Therefore the multiplication map by $\pi$ on $\tilde{R}$ is one-to-one. By flatness of $\hat{R}$, multiplication by $1 \otimes \pi$ is one-to-one on $\ddot{R}$, as claimed.

By (6.7.2), applied to $\tilde{R}$ in place of $\Gamma$, we have $J(\ddot{R})=\ddot{R} \pi$, and the preceding claim showed that $\pi$ is a regular element of $\ddot{R}$. Therefore, if $\ddot{R}$ were noetherian it 
would follow easily that all of its localizations at maximal ideals are integral domains (in fact, discrete valuation rings), and therefore $\ddot{R}$ has no nilpotent elements. On the other hand, $\ddot{R}$ contains the subring $\hat{R} \otimes R=\hat{R}$, which has nilpotent elements because we are assuming that $\tilde{R}$ is not module-finite over $R$ [Mtl '73, 7.1]. This completes the proof of the commutative case.

Now suppose, by way of contradiction, that $\ddot{\Gamma}$ is noetherian. Since $\Gamma$ is modulefinite over $\tilde{R}$, it follows that $\ddot{\Gamma}$ is module-finite over its central subring $\ddot{R}$, and therefore $\ddot{R}$ is noetherian [MR ' $87,10.1 .11$ ], contrary to what was proved above.

(iii) and the rest of (ii). Tensoring the inclusions $R \subseteq \Omega \subseteq \Gamma \subseteq \Omega_{Q}$ with the flat $R$-module $\hat{R}$ yields

$$
\hat{R} \subseteq \hat{\Omega} \subseteq \ddot{\Gamma} \subseteq \hat{\Omega}_{Q}
$$

Recall that the ring $\hat{\Omega}_{Q}$ is artinian [see (3.1.2)]. It follows from (6.7.4) that $N(\ddot{\Gamma})_{Q}$ is a sum of nilpotent ideals of the artinian ring $\hat{\Omega}_{Q}$, and is therefore nilpotent. Therefore (ii) holds.

Let $R^{\prime}, \Omega^{\prime}, \Gamma^{\prime}$ be the natural images of $\hat{R}, \hat{\Omega}, \ddot{\Gamma}$ respectively, in the semisimple artinian ring $A^{\prime}=\hat{\Omega}_{Q} / N\left(\hat{\Omega}_{Q}\right)$. Then the inclusions in (6.7.4) yield:

$$
R^{\prime} \subseteq \Omega^{\prime} \subseteq \Gamma^{\prime} \subseteq A^{\prime} \quad \text { and } \quad\left(\Omega^{\prime}\right)_{Q}=\left(\Gamma^{\prime}\right)_{Q}=A^{\prime} .
$$

We claim that none of these rings has nilpotent ideals $\neq 0$, that is, $R^{\prime}=\hat{R} / N(\hat{R})$, $\Omega^{\prime}=\hat{\Omega} / N(\hat{\Omega})$, and $\Gamma^{\prime}=\ddot{\Gamma} / N(\ddot{\Gamma})$. For any nilpotent ideal $X$ of either $\Omega^{\prime}$ or $\Gamma^{\prime}$, the localization $X_{Q}$ is a nilpotent ideal of $A^{\prime}$ and therefore equals 0 . Hence $X=0$. On the other hand, if $x$ is any nilpotent element of $R^{\prime}$, then centrality of $x$ in $\Omega^{\prime}$ shows that $x A^{\prime}$ is a nilpotent ideal of $A^{\prime}$ and hence $x=0$, proving the claim in this situation, too.

Next we claim that the natural map $R^{\prime} \rightarrow\left(R^{\prime}\right)_{Q}$ (where $Q=Q(R)$ ) is oneto-one, that is, every regular element $d$ of $R$ has a regular image in $R^{\prime}$. Since regular elements of $R$ remain regular in $\hat{R}$ it suffices to show that regular elements of $\hat{R}$ remain regular in $R^{\prime}$. But this follows since, by the previous paragraph, $R^{\prime}=\hat{R} / N(\hat{R})$, and regular elements of a commutative ring remain regular modulo the nil radical.

Although the unadorned $Q$ in (6.7.5) denotes $Q(R)$, we claim that $\left(R^{\prime}\right)_{Q}=$ $\left(R^{\prime}\right)_{Q\left(R^{\prime}\right)}$; that is (since $R^{\prime}$ has no nilpotent elements), $\left(R^{\prime}\right)_{Q}$ is the total quotient ring of $R^{\prime}$. Since $R \subseteq\left(R^{\prime}\right)_{Q}$ by the preceding paragraph, it suffices to show that $\left(R^{\prime}\right)_{Q}$ is artinian. But $\hat{R}_{Q}$ is artinian by $(3.1 .1)$, and $\left(R^{\prime}\right)_{Q}=\hat{R}_{Q} / N(\hat{R})_{Q}$, completing the proof of the claim.

By the claim in the previous paragraph we have $\left(\Omega^{\prime}\right)_{Q\left(R^{\prime}\right)}=\left(\Omega^{\prime}\right)_{Q}=A^{\prime}$, and therefore $\Omega^{\prime}$ is an $R^{\prime}$-order in the semisimple artinan ring $A^{\prime}$.

Moreover, the semilocal ring $R^{\prime}$ is complete in its $J\left(R^{\prime}\right)$-adic topology. [Proof. This topology coincides with the $J(\hat{R})$-adic topology of $R^{\prime}$, and the completion of $R^{\prime}$ in this topology is $\hat{R} \otimes_{\hat{R}} R^{\prime}=R^{\prime}$.]

Next we claim that $\Gamma^{\prime}$ is integral over $R^{\prime}$. It suffices to prove that $\Gamma^{\prime}$ is a set-theoretic union of module-finite $R^{\prime}$-algebras. And for this it suffices to prove that $\ddot{\Gamma}$ is a set-theoretic union of module-finite $\hat{R}$-algebras. Since tensor products commute with direct limits, it suffices to show that $\Gamma$ is a directed set-theoretic union of module-finite $R$-algebras; and this is proved in (4.1.1).

Next we claim that $\Gamma^{\prime}$ is a module-finite $R^{\prime}$-algebra. Since $\Gamma^{\prime}$ is integral over $R^{\prime}$, a simple application of Zorn's Lemma shows that some normalization $\Gamma^{\prime \prime}$ of 
$\Omega^{\prime}$ contains $\Gamma^{\prime}$. (In fact, when the proof of the lemma is complete, we will know that $\Gamma^{\prime \prime}=\Gamma^{\prime}$.) Since $R^{\prime}$ is complete in its $J\left(R^{\prime}\right)$-adic topology, it follows from Proposition 4.4 that $\Gamma^{\prime \prime}$ is a f.g. $R^{\prime}$-module, hence so is $\Gamma^{\prime}$, as claimed.

Since $R^{\prime} \cong \hat{R} / N(\hat{R})$ and $\Gamma^{\prime} \cong \ddot{\Gamma} / N(\ddot{\Gamma})$, statement (iii) of our lemma is now proved, except for the assertion that the $R^{\prime}$-order $\Gamma^{\prime}$ is a maximal order.

Let $\delta$ be as in statement (ii), and $\delta^{\prime}$ its image in $\Gamma^{\prime}$. We claim that $\delta^{\prime}$ is a regular element of $\Gamma^{\prime}$.

Since $N(\ddot{\Gamma}) \subseteq J(\ddot{\Gamma})$ it follows from statement (ii) that $J\left(\Gamma^{\prime}\right)=\Gamma^{\prime} \delta^{\prime}$. As in the proof of (6.7.2), let $c$ be a regular element in $J(R)$. Then the natural image $c^{\prime}$ of $c$ in $R^{\prime}$ is a non-zero-divisor in $\Gamma^{\prime}$, as proved two paragraphs below (6.7.5). Also, it follows from (6.7.1) that $c^{\prime} \in J\left(\Gamma^{\prime}\right)$. Since $J\left(\Gamma^{\prime}\right)=\Gamma^{\prime} \delta^{\prime}$ contains the non-zerodivisior $c^{\prime}$, it follows that $\delta^{\prime}$ is not a left zero divisor in $\Gamma^{\prime}$, and therefore in $\left(\Gamma^{\prime}\right)_{Q}$. Since $\left(\Gamma^{\prime}\right)_{Q}$ is an artinian ring, $\delta^{\prime}$ is therefore a unit in $\left(\Gamma^{\prime}\right)_{Q}$ and therefore a regular element of $\Gamma^{\prime}$, as claimed.

Next we claim that $\Gamma^{\prime}$ is a hereditary $R^{\prime}$-order (i.e., all left and right ideals are projective $\Gamma^{\prime}$-modules). Since $\delta^{\prime}$ is a regular element of $\Gamma^{\prime}$, the left ideal $J\left(\Gamma^{\prime}\right)=\Gamma^{\prime} \delta^{\prime}$ is a free, hence projective $\Gamma^{\prime}$-module. In the situation that $R^{\prime}$ is a discrete valuation ring, a well-known theorem of Auslander and Goldman states that our claim is true. In fact, the proof of this result given in [ $\mathrm{R}$ '75, (39.1)] works in our more general situation, with the following very minor change. Instead of choosing the element $\pi$, in that proof, to be a generator of $J\left(R^{\prime}\right)$, which might not be principal in our situation, choose it to be the regular element $c^{\prime} \in J\left(R^{\prime}\right)$. The proof then works without additional changes.

Since $\Gamma^{\prime}$ is a hereditary, noetherian semiprime ring, it is easy to see that $\Gamma^{\prime}$ is a direct sum of hereditary noetherian prime rings [MR '87, 5.4.6]. Since $\Gamma^{\prime}$ is modulefinite over a commutative ring, it satisfies a polynomial identity. Therefore, by a theorem of Robson and Small [MR '87, 13.9.16], [RS '74], the centers of the prime summands of $\Gamma^{\prime}$ are Dedekind domains (complete discrete valuation rings, in our situation), and therefore $\Gamma^{\prime}$ is a direct sum of hereditary classical orders. It now suffices to show that all of these prime summands are maximal orders.

The structure theorem for hereditary orders over complete discrete valuation rings in simple algebras [ $\left.\mathrm{R}{ }^{\prime} 75,(39.14)\right]$ states that every such order is isomorphic to a tiled block lower pseudo-triangular matrix ring of the form

$$
T=\left[\begin{array}{ccccc}
(\Delta) & (\mathbf{p}) & (\mathbf{p}) & \ldots & (\mathbf{p}) \\
(\Delta) & (\Delta) & (\mathbf{p}) & \ldots & (\mathbf{p}) \\
(\Delta) & (\Delta) & (\Delta) & \ldots & (\mathbf{p}) \\
\vdots & \vdots & \vdots & \ddots & \vdots \\
(\Delta) & (\Delta) & (\Delta) & \ldots & (\Delta)
\end{array}\right]^{\left\{n_{1}, \ldots, n_{r}\right\}}
$$

which we now explain. The superscript $\left\{n_{1}, \ldots, n_{r}\right\}$ denotes a sequence of $r \geq 1$ positive integers $n_{i}$. For each $(i, j)$, the symbol $(\Delta)$ or $(\mathbf{p})$ in the $(i, j)$-position of the matrix in (6.7.6) indicates the set of all $n_{i} \times n_{j}$ matrices with enries in $(\Delta)$ or (p) respectively. Moreover, $\Delta$ denotes a maximal $R^{\prime}$-order in a division algebra, and $\mathbf{p}=J(\Delta)$.

The structure theorem quoted above also states that $T$ is a maximal order if and only if $r=1$, that is, $T=M_{n_{1}}(\Delta)$.

It is easy to see that one can form $J(T)$ by changing every $n_{i} \times n_{i}$ ("main diagonal") block from $(\Delta)$ to $(\mathbf{p})$. Note that the only central idempotents of $T$ 
are $\{0,1\}$. Therefore, if $r \neq 1$, then $T / J(T)$ has central idempotents that cannot be lifted to central idempotents of $T$. We conclude: If every primitive central idempotent of every $T / J(T)$ can be lifted to a central idempotent of $T$, then $\Gamma^{\prime}$ is a maximal order.

We now verify that every primitive central idempotent of $\Gamma^{\prime} / J\left(\Gamma^{\prime}\right)$ can be lifted to a central idempotent of $\ddot{\Gamma}$.

We have $\Gamma^{\prime} / J\left(\Gamma^{\prime}\right) \cong \ddot{\Gamma} / J(\ddot{\Gamma}) \cong \Gamma / J(\Gamma)$, the last isomorphism by (6.7.3). Therefore, to complete the proof of the lemma it suffices to verify that every primitive central idempotent of $\bar{\Gamma}=\Gamma / J(\Gamma)$ can be lifted to a central idempotent of $\ddot{\Gamma}$.

Let $\Omega$ be any splitting $R$-order contained in $\Gamma$. We have $\bar{\Gamma}=\Omega / J(\Omega)=\hat{\Omega} / J(\hat{\Omega})$ by (6.1.4) and Proposition 3.8. It is obvious, from Lemma 6.5 , that every indecomposable ring-direct summand of $\hat{\Omega} / J(\hat{\Omega})$ is the image of an indecomposable ring-direct summand of $\hat{\Omega}$. This is equivalent to saying that every primitive central idempotent of the former ring can be lifted to a primitive central idempotent of the latter ring. In view of inclusions (6.7.4), our proof is complete.

The next results show that primitive idempotents of $\hat{\Omega}$ remain primitive in $\hat{\Omega}_{Q}$. The commutative case of this was proved by D. Katz [Kt '86], but from a somewhat different point-of-view. Katz's results, in dimension 1 , are that if $R$ is a local domain, then the kernel of the natural map $\hat{R} \rightarrow(\tilde{R})^{\wedge}$ is nilpotent and both rings have the same number of minimal prime ideals, namely, the number of maximal ideals of $\tilde{R}$. Our proof is an adaptation of Katz's proof, but must be modified to avoid his use of integral closures of ideals (which do not exist in our noncommutative setting), and to accomodate the presence of non-central idempotents (which are not related to prime ideals). We are grateful to W. Heinzer for informing us about Katz's work, and to L. Klingler for a key idea that enabled us to use this alternative approach. See also Remark 6.10.

Lemma 6.8. The kernel of the natural map $\nu: \hat{\Omega} \rightarrow \hat{\Gamma}$ is nilpotent, where $\hat{\Gamma}$ denotes the $J(\tilde{R})$-adic completion of $\Gamma$.

Note. To see that the kernel can be nonzero, consider the simplest commutative situation, in which $R=\Omega$ and is a local domain whose normalization $\tilde{R}=\Gamma$ is again local (hence a discrete valuation ring) but not module-finite over $R$. Then the $J(R)$-adic completion $\hat{R}$ of $R$ contains nilpotent elements [Mtl '73, 7.1], while the $J(\tilde{R})$-adic completion $\hat{\Gamma}$ of $\tilde{R}$ is a local domain, in fact a discrete valuation ring. Then the natural map in the statement of the lemma is clearly a nonzero nilpotent ideal of $\hat{R}=\hat{\Omega}$.

Proof of the lemma. We may assume that $\Lambda=\Omega$, since $\Lambda$ itself does not appear in the statement of the lemma. We also may assume that $R=Z(\Omega)$, by Lemma 6.2. By Lemma 1.3(iii), $J(R)$ contains regular elements of $R$. Fix such an element $a$ for the rest of this proof. Since $R / R a$ is an artinian ring, each of the ideals $R a$ and $J(R)$ contains some power of the other; and therefore the $J(R)$-adic completion $\hat{R}$ can be identified with the completion of $R$ with respect to the filter of ideals $\left\{R a^{n}: n=1,2, \ldots\right\}$. Consequently $\hat{\Omega}$ can be identified with the completion of $\Omega$ with respect to the filter of ideals $\left\{\Omega a^{n}\right\}$.

Since $\tilde{R}$ is integral over $R$, the commutative lying-over and going-up theorems show that $a \in J(\tilde{R})$; and since $a$ is regular in $R$ it is a unit in $R_{Q}$ and therefore 
regular in $\tilde{R}$. We conclude, as in the previous paragraph, that the $J(\tilde{R})$-adic completion $\hat{\Gamma}$ can be identified with the completion of $\Gamma$ with respect to the filter of ideals $\left\{\Gamma a^{n}\right\}$.

Let $\Gamma^{\prime}=\ddot{\Gamma} / N(\ddot{\Gamma})$, as in (6.7.5) and the paragraph below it, and let $a^{\prime}$ be the natural image of $a$ in $\Gamma^{\prime}$. We claim that

$$
\bigcap_{n=1}^{\infty} \Gamma^{\prime}\left(a^{\prime}\right)^{n}=0
$$

Call this intersection $X$. Since $a$ is a unit in $\Omega_{Q}$, hence in $\hat{\Omega}_{Q}, a^{\prime}$ is a unit in $A^{\prime}=\hat{\Omega}_{Q} / N\left(\hat{\Omega}_{Q}\right)$ and hence is a non-zero-divisor on $\Gamma^{\prime}$. Therefore $X=a^{\prime} X$. Since $\Gamma^{\prime}$ is a f.g. module over the noetherian ring $\hat{R} / N(\hat{R})$, by Lemma $6.7(\mathrm{iii})$, and $a^{\prime} \in J\left(R^{\prime}\right)$, it follows from Nakayama's Lemma that $X=0$, as claimed.

We now apply our adaptation of Katz's main step [Kt '86, last paragraph of proof of Theorem 4].

Choose any element $x^{*} \in \operatorname{ker}(\nu)$. We have $x^{*}=\lim _{n} x_{n}$ for some Cauchy sequence $\left\{x_{n}\right\}$ in $\Omega$. After passing to a suitable subsequence we have $x^{*}-x_{n} \in \hat{\Omega} a^{n}$ for every $n$. On the other hand, $\nu\left(x^{*}\right)=0$ shows that $\left\{x_{n}\right\}$ is a null sequence in $\Gamma$. Therefore, after passing to a suitable subsequence, we have $x_{n} \in \Gamma a^{n}$ for every $n$.

Note that $\Gamma$ and $\hat{\Omega}$ are subrings of $\ddot{\Gamma}$. Therefore we may write $x^{*}=x_{n}+\left(x^{*}-x_{n}\right)$, obtaining an expression of the form

$$
x^{*}=x_{n}+y_{n} \quad \text { with }\left\{\begin{array}{l}
x_{n} \in \Gamma a^{n}, \\
y_{n} \in \hat{\Omega} a^{n},
\end{array}\right.
$$

for every $n$. Therefore $x^{*} \in \ddot{\Gamma} a^{n}$ for every $n$. It follows from (6.8.1) that the image of $x^{*}$ in $\Gamma^{\prime}$ equals zero. Therefore

$$
x^{*} \in \operatorname{ker}\left(\ddot{\Gamma} \rightarrow \Gamma^{\prime}\right)=N(\ddot{\Gamma}) .
$$

Since $N(\ddot{\Gamma})$ is a nilpotent ideal [by Lemma 6.7 ] we conclude that $\operatorname{ker}(\nu)$ is nilpotent.

Theorem 6.9. Primitive idempotents of $\hat{\Omega}$ remain primitive in $\hat{\Omega}_{Q}$ (hence in its subring $\ddot{\Gamma}$ ) and $\hat{\Gamma}_{Q}$ (hence in its subring $\hat{\Gamma}$ ).

Proof. First we recall that $\ddot{\Gamma}$ is indeed a subring of $\hat{\Omega}_{Q}$, as shown in inclusions (6.7.4).

Next we note that primitive idempotents of $\hat{\Gamma}$ remain primitive in $\hat{\Gamma}_{Q}$. This is a restatement of a well-known fact about classical orders. For $\Gamma$ is a maximal $\tilde{R}$-order and is therefore a direct sum of maximal orders, over principal ideal domains, in simple algebras. [See (4.1.2) and (4.1.3).] It follows that $\hat{\Gamma}$ is a direct sum of full matrix rings over valuation rings in division rings. See [ $\mathrm{R}^{\prime} 75$, bottom of p. 170]. The claim now follows easily.

Let $d$ be a primitive idempotent in $\hat{\Omega}$. We claim that $d$ remains primitive in $\hat{\Gamma}$. If we knew that the natural map $\hat{\Omega} \rightarrow \hat{\Gamma}$ is a surjection, this would follow from nilpotence of its kernel. Instead, we proceed less directly. By completeness, an idempotent $d$ of $\hat{\Omega}$ is primitive in $\hat{\Omega}$ if and only its natural image in $\hat{\Omega} / J(\hat{\Omega})$ is primitive there (because idempotents can be lifted modulo the radical, in the complete case $[\mathrm{CR}$ ' $81,(6.7)])$. Since $\Omega$ is a splitting order, we have $\Omega / J(\Omega)=$ $\Gamma / J(\Gamma)$, and hence $\hat{\Omega} / J(\hat{\Omega})=\hat{\Gamma} / J(\hat{\Gamma})$. Thus, if $d$ is primitive in $\hat{\Omega}$, it remains 
primitive in $\hat{\Omega} / J(\hat{\Omega})=\hat{\Gamma} / J(\hat{\Gamma})$, and hence remains primitive in $\hat{\Gamma}$. Therefore, by the previous paragaph, $d$ also remains primitive in $\hat{\Gamma}_{Q}$.

Now suppose that $d=e+f$ with $e, f$ orthogonal, nonzero idempotents in $\hat{\Omega}_{Q}$ and $d$ primitive in $\hat{\Omega}$. Let $\nu$ denote both the natural homomorphism $\hat{\Omega} \rightarrow \hat{\Gamma}$ and its extension $\nu_{Q}: \hat{\Omega}_{Q} \rightarrow \hat{\Gamma}_{Q}$. Then we have $\nu(d)=\nu(e)+\nu(f)$, where the terms on the right-hand side are orthogonal idempotents of $\hat{\Gamma}_{Q}$. Since $\operatorname{ker}(\hat{\Omega} \rightarrow \hat{\Gamma})$ is a nilpotent ideal [Lemma 6.8], so is $\operatorname{ker}\left(\hat{\Omega}_{Q} \rightarrow \hat{\Gamma}_{Q}\right)$. Therefore both terms $\nu(e), \nu(f)$ are nonzero. But, as shown above, $\nu(d)$ is primitive in $\hat{\Gamma}$, and hence in $\hat{\Gamma}_{Q}$, a contradiction. Therefore $d$ remains primitive in $\hat{\Omega}_{Q}$.

Remark 6.10. We now know as much as we need to know about $\hat{\Omega}$ versus $\hat{\Gamma}$ versus $\ddot{\Gamma}$, in order to obtain our package deal theorem for completions. But our results are not completely satisfying. By passing to the direct limit, the natural maps $\hat{\Omega} \rightarrow \hat{\Gamma}$ induce a map $\ddot{\Gamma} \rightarrow \hat{\Gamma}$ whose kernel is nilpotent by Lemma 6.8. Is this map a surjection? If so, then the maximal order $\ddot{\Gamma} / N(\ddot{\Gamma})$ in Lemma 6.7 can presumably be identified with $\hat{\Gamma}$. If the map is not a surjection, is the induced map $\ddot{\Gamma}_{Q} \rightarrow \hat{\Gamma}_{Q}$ of artinian rings a surjection?

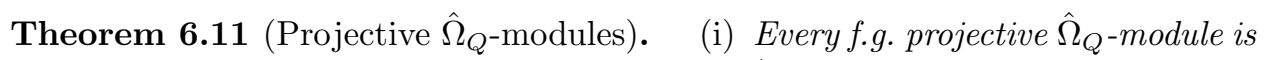
isomorphic to $U_{Q}$ for some f.g. projective $\hat{\Omega}$-module $U$ that is unique up to isomorphism.

(ii) (In more detail). Let $U_{k i}$ be the unique indecomposable f.g. projective $\hat{\Omega}_{k}$ module that maps onto the simple module $S_{k i}$, as in Theorem 6.6. Then the indexed set of modules $\left(U_{k i}\right)_{Q}$ forms a set of representatives for the isomorphism classes of indecomposable f.g. projective $\hat{\Omega}_{Q}$-modules.

Proof. Write the identity element of $\hat{\Omega}$ as a sum $1=\sum_{i} e_{i}$ of orthogonal primitive idempotents $e_{i}$. Since the Krull-Schmidt theorem holds for f.g. modules over the complete ring $\hat{\Omega}$, every indecomposable f.g. projective $\hat{\Omega}$-module is isomorphic to some $\hat{\Omega} e_{i}$. These idempotents $e_{i}$ remain primitive in $\hat{\Omega}_{Q}$ [Theorem 6.9]; and since the Krull-Schmidt theorem holds for f.g. modules over the artinian ring $\hat{\Omega}_{Q}$, we see that every indecomposable f.g. projective $\hat{\Omega}_{Q}$-module is isomorphic to some $\hat{\Omega}_{Q} e_{i}=\left(\hat{\Omega} e_{i}\right)_{Q}$.

Thus it suffices to show that if indecomposable f.g. projective $\hat{\Omega}$-modules satisfy $U \neq V$, then we have $U_{Q} \neq V_{Q}$. This follows from Theorem 6.6 and Lemma 6.5, which show that $U \cong U_{k i}$ and $V \cong U_{k^{\prime} i^{\prime}}$ where $U_{k i}$ and $U_{k^{\prime} i^{\prime}}$ are isomorphic to left ideals of distinct ring-direct summands of $\hat{\Omega}$, hence of $\hat{\Omega}_{Q}$.

Proposition 6.12. $\ddot{\Gamma}$ is a semiperfect ring.

Proof. We need to show that the ring $\ddot{\Gamma}$ becomes artinian modulo $J(\ddot{\Gamma})$ and idempotents of $\ddot{\Gamma} / J(\ddot{\Gamma})$ can be lifted to idempotents of $\ddot{\Gamma}$. We may assume that $R=Z(\Lambda)$.

Let $\bar{\Gamma}=\Gamma / J(\Gamma)$, and recall that $\Gamma / J(\Gamma)=\ddot{\Gamma} / J(\ddot{\Gamma})$ by (6.7.3). It follows that the ring $\ddot{\Gamma}$ becomes artinian modulo $J(\ddot{\Gamma})$. Thus it suffices to show that every idempotent $\bar{e} \in \bar{\Gamma}$ can be lifted to an idempotent $e \in \ddot{\Gamma}$.

Choose any splitting $R$-order $\Omega \subseteq \Gamma$. Then $\bar{\Gamma}=\Omega / J(\Omega)=\hat{\Omega} / J(\hat{\Omega})$, the first equality by (6.1.4) and the second by (6.1.7). By completeness of $\hat{\Omega}, \bar{e}$ can be lifted to an idempotent $e \in \hat{\Omega}$. But, by flatness of $\hat{R}$ as an $R$-module, $\hat{\Omega}=\hat{R} \otimes_{R} \Omega$ is 
a subring of $\ddot{\Gamma}=\hat{R} \otimes_{R} \Gamma$, and $e$ is therefore the desired idempotent of $\ddot{\Gamma}$ that lifts $\bar{e}$.

By (6.7.3) we have $\ddot{\Gamma} / J(\ddot{\Gamma})=\bar{\Gamma}$. Therefore the simple $\Gamma$-modules $S_{k i}$ form a set of representatives of the isomorphism classes of simple (left) $\ddot{\Gamma}$-modules.

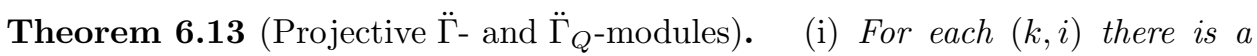
unique indecomposable f.g. projective $\ddot{\Gamma}$-module $\ddot{U}_{k i}$ that can be mapped onto $S_{k i}$. The indexed family of modules $\ddot{U}_{k i}$ forms a set of representatives of the isomorphism classes of indecomposable, f.g. projective $\ddot{\Gamma}$-modules.

(ii) The indexed family of modules $\left(\ddot{U}_{k i}\right)_{Q}$ forms a set of representatives of the isomorphism classes of indecomposable f.g. projective $\ddot{\Gamma}_{Q}$-modules.

(iii) Let $\Omega$ be a splitting $R$-order $(\Omega \subseteq \Gamma)$ and let $U_{k i}$ be the indecomposable f.g. projective $\hat{\Omega}$-module that maps onto $S_{k i}$, as in Theorem 6.6. Then $\left(U_{k i}\right)_{Q} \cong$ $\left(\ddot{U}_{k i}\right)_{Q}$.

Proof. (i). Since $\bar{\Gamma}$ is a semisimple artinian ring, each $S_{k i} \cong \bar{\Gamma} \bar{e}_{k i}$ for some primitive idempotent $\bar{e}_{k i} \in \bar{\Gamma}$. Since $\bar{\Gamma}=\ddot{\Gamma} / J(\ddot{\Gamma})$ and $\ddot{\Gamma}$ is a semiperfect ring [Proposition 6.12], each $\bar{e}_{k i}$ lifts to a primitive idempotent $e_{k i}$ of $\ddot{\Gamma}$, and the indexed family of modules $\ddot{U}_{k i}=\ddot{\Gamma} e_{k i}$ forms a set of representatives of the isomorphism classes of indecomposable f.g. projective $\ddot{\Gamma}$-modules.

(ii). Write $\ddot{\Gamma}=\bigoplus_{\nu} \ddot{\Gamma} e_{\nu}$ where the $e_{\nu}$ are primitive orthogonal idempotents with $\sum_{\nu} e_{\nu}=1$. Then we have $\ddot{\Gamma}_{Q}=\bigoplus_{\nu} \ddot{\Gamma}_{Q} e_{\nu}$. Since primitive idempotents of $\ddot{\Gamma}$ remain primitive in the artinian ring $\ddot{\Gamma}_{Q}$ [Theorem 6.9], every isomorphism class of indecomposable projective $\ddot{\Gamma}_{Q}$-module is isomorphic to some term $\ddot{\Gamma}_{Q} e_{\nu}$. Thus it suffices to show that non-isomorphic terms $\ddot{\Gamma} e_{\nu}$ remain non-isomorphic after $Q$ localization; and to show this it suffices to show that non-isomorphic terms $\ddot{\Gamma} e_{\nu}$ are contained in distinct ring-direct summands of $\ddot{\Gamma}_{Q}$. To do this, note that every finite set of elements of $\ddot{\Gamma}=\hat{R} \otimes_{R} \Gamma$ is contained in a ring of the form $\hat{\Omega}=\hat{R} \otimes_{R} \Omega$ where $\Omega$ is a splitting $R$-order contained in $\Gamma$ [Theorem 5.2]. Therefore we can choose a splitting order $\Omega$ such that $\hat{\Omega}$ contains every $e_{\nu}$. By Lemma 6.5 non-isomorphic $\hat{\Omega}$ modules $\hat{\Omega} e_{\nu}$ are left ideals of distinct ring-direct summands of $\hat{\Omega}$. Therefore their $Q$-localizations $\left(\hat{\Omega} e_{\nu}\right)_{Q}=\left(\ddot{\Gamma} e_{\nu}\right)_{Q}$ are contained in distinct ring-direct summands of $\hat{\Omega}_{Q}=\ddot{\Gamma}_{Q}$, as desired.

(iii) Let $S_{k i} \cong \bar{\Gamma} \bar{e}_{k i}$, as in the proof of part (i). Since $\hat{\Omega}$ is complete, $\bar{e}_{k i}$ lifts to a primitive idempotent $e_{k i} \in \hat{\Omega}$. Since $\hat{\Omega} e_{k i}$ is indecomposable and maps onto $S_{k i}$, the uniqueness part of Theorem 6.6(i) shows that $U_{k i} \cong \hat{\Omega} e_{k i}$. Since $\hat{\Omega}=\hat{R} \otimes_{R} \Omega$ is a subring of $\ddot{\Gamma}, e_{k i}$ is also an idempotent in $\ddot{\Gamma}$. Moreover, since $\ddot{\Gamma}$ is semiperfect and (6.7.3) holds, $e_{k i}$ is a primitive idempotent of $\ddot{\Gamma}$. Therefore our notation $e_{k i}$ is consistent with its use in the proof of part (i). In particular, $\ddot{U}_{k i} \cong \ddot{\Gamma} e_{k i}$. But $\hat{\Omega}_{Q}=\ddot{\Gamma}_{Q}$, as one sees by localizing the inclusions $\Omega \subseteq \Gamma \subseteq \Gamma_{Q}=\Omega_{Q}$ at $Q$ and then tensoring with $\hat{R}$. Therefore $\left(\hat{\Omega} e_{k i}\right)_{Q}=\left(\ddot{\Gamma} e_{k i}\right)_{Q}$, completing the proof of part (iii).

Notation 6.14 (Ranks). Let $X$ be a f.g. $\hat{\Lambda}$-module such that the $\hat{\Lambda}_{Q^{-}}$-module $X_{Q}$ is projective. We have $\ddot{\Gamma}_{Q}=\hat{\Lambda}_{Q}$, as one can see by localizing the inclusions $\Lambda \subseteq \Gamma \subseteq$ $\Lambda_{Q}$ at $Q$ and then tensoring with $\hat{R}$. Since the ring $\ddot{\Gamma}_{Q}=\hat{\Lambda}_{Q}$ is artinian [see (3.1.2)] we can express $X_{Q}$ uniquely as a direct sum of indecomposable projective $\ddot{\Gamma}_{Q^{-}}$ modules. Thus, in the notation of Theorem 6.13(ii), there exist unique nonnegative 
integers $\rho_{k i}(X)$ such that

$$
X_{Q} \cong \bigoplus_{k i}\left[\left(\ddot{U}_{k i}\right)_{Q}\right]^{\rho_{k i}(X)} .
$$

We call the array $\rho(X)=\left(\left(\rho_{k i}\right)\right)=\left(\left(\rho_{k i}(X)\right)\right)$ the array of ranks of $X$.

Alternatively, this definition can be phrased in terms of splitting orders. Let $\Omega$ be a splitting $R$-order $(\Omega \subseteq \Gamma)$. There is a unique indecomposable projective $\hat{\Omega}$-module $U_{k i}$ such that $\left(U_{k i}\right)_{Q} \cong\left(\ddot{U}_{k i}\right)_{Q}$ [Theorem 6.13(iii)]. If one wishes to avoid mentioning $\ddot{\Gamma}$-modules, $U_{k i}$ can be described as the unique indecomposable f.g. projective $\hat{\Omega}$-module that maps onto the simple module $S_{k i}$ (over any of the rings $\Omega, \hat{\Omega}, \Gamma, \bar{\Gamma})$. Then $(6.14 .1)$ can be rewritten:

$$
X_{Q} \cong \bigoplus_{k i}\left[\left(U_{k i}\right)_{Q}\right]^{\rho_{k i}(X)} .
$$

Note that the array of ranks $\rho(X)$ is not a matrix, because its rows need not all have the same length. The subscript $Q$ denotes $Q(R)$, as usual.

Package Deal Theorem 6.15 (Completions of orders). Let $X$ be a f.g. left $\hat{\Lambda}$ module and $\Omega$ a splitting $R$-order $(\Omega \subseteq \Gamma)$. Then the following conditions are equivalent.

(i) $X \cong \hat{A}$ for some f.g. $\Lambda$-module $A$.

(ii) $X_{Q}$ is $\hat{\Lambda}_{Q}$-projective and, for every $k$, the array of ranks $\rho(X)=\left(\left(\rho_{k i}\right)\right)$ satisfies

$$
\left(\rho_{k 1}, \rho_{k 2}, \ldots\right) \in \mathbb{N} \cdot\left(s_{k 1}, s_{k 2}, \ldots\right)
$$

where $\mathbb{N}$ denotes the nonnegative integers, and $s_{k 1}, s_{k 2}, \ldots$ are the splitting numbers of $\Gamma$, as defined in (6.1.2) and (6.1.3).

(iii) $X_{Q} \cong \hat{P}_{Q}$ for some f.g. projective $\Omega$-module P. [Alternatively: $X_{Q} \cong \hat{R} \otimes N_{Q}$ for some f.g. projective $\Gamma$-module $N$.]

Proof. (i) $\Rightarrow$ (iii). Condition (i) implies that $X_{Q} \cong \hat{A}_{Q}=\hat{R} \otimes_{R} A \otimes_{R} R_{Q}$. Since $\Lambda_{Q}$ is a semisimple artinian ring, the $\Lambda_{Q}$-module $A \otimes_{R} R_{Q}$ is projective. It now suffices to prove that every f.g. (necessarily projective) $\Lambda_{Q}$-module is isomorphic to $P_{Q}$ for some f.g. projective $\Omega$-module $P$. This holds since $\Omega$ is a splitting order [Theorem 5.5].

To obtain the alternative form of (iii), first recall that $\Omega \subseteq \Gamma \subseteq \Omega_{Q}$ and hence $\Omega_{Q}=\Gamma_{Q}$. Therefore it suffices to show that if we are given a f.g. projective $\Omega$ module $P$ there exists a f.g. projective $\Gamma$-module $N$ such that $P_{Q} \cong N_{Q}$; and conversely, if $N$ is given then $P$ exists such that $P_{Q} \cong N_{Q}$.

Given $P$, let $N=\Gamma \otimes_{\Omega} P$. Conversely, suppose $N$ is given. Then $N_{Q}$ is a f.g. projective $\Gamma_{Q}=\Omega_{Q}$-module, and therefore Theorem 5.5 yields $P$ such that $P_{Q} \cong N_{Q}$.

(iii) $\Rightarrow$ (ii). Write $P$ as a direct sum of copies of the indecomposable projective $\Omega$-modules $B_{k}$, yielding an expression $P=\bigoplus_{k}\left(B_{k}\right)^{m_{k}}$. Take the completion, and substitute $\hat{B}_{k}=\bigoplus_{i}\left(U_{k i}\right)^{s_{k i}}$ [Theorem 6.6]. Then localize at $Q$. This yields

$$
X_{Q} \cong \hat{P}_{Q} \cong \bigoplus_{k i}\left(\left(U_{k i}\right)_{Q}\right)^{m_{k} s_{k i}} .
$$

Therefore $X$ has the array of ranks whose $k^{\text {th }}$ row is $m_{k}\left(s_{k 1}, s_{k 2}, \ldots\right)$, as required. 
(ii) $\Rightarrow$ (i). By (6.15.1) we can let $\rho_{k i}=m_{k} s_{k i}$ for all $k, i$. Set $P=\bigoplus_{k}\left(B_{k}\right)^{m_{k}}$, a projective $\Omega$-module. Then, by Theorem $6.6, \hat{P} \cong \bigoplus_{k i}\left(U_{k i}\right)^{m_{k} s_{k i}}$, and therefore $\hat{P}_{Q} \cong X_{Q}$. Therefore, by the abstract form of the package deal theorem for completions, Theorem 3.4, $X$ is the $J(R)$-adic completion of some f.g. $\Lambda$-module.

Corollary 6.16. Suppose that

(i) Every row of the array of splitting numbers of $\Gamma$ consists of the single number $s_{k 1}$, and $s_{k 1}=1$.

Then:

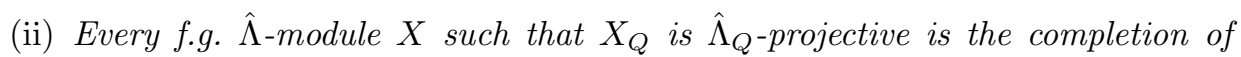
some f.g. $\Lambda$-module.

This, in turn, implies that:

(iii) The Krull-Schmidt theorem holds for f.g. $\Lambda$-modules.

Proof. (i) $\Rightarrow$ (ii). This is the trivial case of implication (ii) $\Rightarrow$ (i) of Theorem 6.15 .

(ii) $\Rightarrow$ (iii). (This observation is credited to I. Reiner in [H '61].) The KrullSchmidt theorem holds for f.g. modules over the complete ring $\hat{\Lambda}$ [CR '81, (6.12)]. Since f.g. $\Lambda$-modules are isomorphic if and only if their completions are isomorphic $\hat{\Lambda}$-modules, the Krull-Schmidt theorem therefore descends to f.g. $\Lambda$-modules when condition (ii) holds.

Remarks 6.17. (i) A less technical restatement of condition (i) of Corollary 6.16 is:

(6.17.1) Every $\Gamma_{k}$ is matrix-local [i.e. $\Gamma_{k} / J\left(\Gamma_{k}\right)$ is a simple artinian ring] and idempotents of $\Gamma_{k} / J\left(\Gamma_{k}\right)$ can be lifted to $\Gamma_{k}$.

Yet another equivalent condition is:

(6.17.2) (Heller's condition). The $J(R)$-induced completion of every simple (left)

$\Gamma_{Q}$-module is a simple $\hat{\Gamma}_{Q}$-module.

To prove this latter equivalence, first note that the $J(R)$-adic completion of any $\Gamma$ module is the same as its $J(\tilde{R})$-adic completion [see the paragraphs before (6.1.7)]; and similarly, the $J(R)$-induced completion of any $\Gamma_{Q}$-module is the same as its $J(\tilde{R})$-induced completion. [For the definition of " $J(R)$-induced completion", see either the comments about it in the discussion of $\S 3$ in the Introduction to this paper, or else the cautionary statement below statement (3.1.1)] Moreover, $\Gamma$ is a direct sum of classical maximal orders over semilocal principal ideals domains (namely, the indecomposable ring-direct summands of $\tilde{R}$ ), by (4.1.3). Therefore $\hat{\Gamma}_{Q}$ is a semisimple artinian ring, and therefore the simple $\hat{\Gamma}_{Q}$-modules in Heller's condition coincide with the indecomposable projective $\hat{\Gamma}_{Q}$-modules.

In the situation $R=\tilde{R}$ in which we may now work, $\Gamma$ is a maximal, hence splitting $R$-order. Hence we may take $\Omega=\Gamma$ in Theorem 6.6. Then Theorem 6.6 shows that we have $\hat{B}_{k} \cong \bigoplus_{i}\left(U_{k i}\right)^{s_{k i}}$ where $B_{k}$ denotes (in this situation) the indecomposable projective $\Gamma_{k}$-module and the $U_{k i}$ denote the indecomposable projective $\hat{\Gamma}_{k}$-modules. Since each $\left(U_{k i}\right)_{Q}$ is an indecomposable $\Gamma_{Q}$-module [by Theorem 6.11], our claim is now obvious, by comparing Heller's condition with condition (i) of Corollary 6.16. [Of course, one does not need the complicated machinery of the present paper to prove the present claim about classical orders.]

(ii) (Heller's theorem). The implication (6.17.2) $\Rightarrow$ Corollary 6.16(iii) generalizes a well-known theorem of Heller. His original result assumes that $\Lambda$ is contained in a 
maximal $R$-order in $\Lambda_{Q}$ (i.e. $\Gamma$ is module-finite over $R$ ) and $R$ is a discrete valuation ring of characteristic zero. The substance (but not statement) of this is contained in [H '61, paragraph before 2.5 and proof of 2.6]. The "characteristic zero" restriction was removed in [CR '81, (30.18)], but only for torsionfree modules.

In $[\mathrm{LO}]$ we refine Heller's theorem in such a way that it provides a necessary and sufficient condition for the Krull-Schmidt Theorem to hold for f.g. $\Lambda$-modules.

(iii) If $\Gamma$ is a f.g. $R$-module, the statements of both Theorem 6.15 and Corollary 6.16 can be simplified by not mentioning that $X_{Q}$ is $\hat{\Lambda}_{Q}$-projective [because the ring $\hat{\Lambda}_{Q}$ is semisimple artinian, and hence all of its modules are projective] and by setting $\Omega=\Gamma$. In this form the theorem is well-known in integral representation theory, although we do not know a precise reference to it.

\section{ARTINIAN PAIRS}

We now compare the preceding package deal theorem with its counterpart involving artinian pairs. This counterpart has the advantage of being simpler to apply, because it avoids the cumbersome machinery of completions. But it applies only to torsionfree $\Lambda$-modules.

Notation 6.18. Let $\Lambda \subseteq \Omega \subseteq \Lambda_{Q}$, where $\Lambda$ is an $R$-order in the semisimple ring $\Lambda_{Q}$ and $\Omega$ is a splitting $R$-order, and $R$ is semilocal. Let $C \subseteq J(\Lambda) \cap J(\Omega)$ be a conductor ideal for $\Lambda$ and $\Omega$; that is, an ideal of both rings such that $\bar{\Lambda}=\Lambda / C$ and $\bar{\Omega}=\Omega / C$ are $R$-modules of finite length, and therefore artinian rings. Since we are assuming that $Z(\Lambda)$ has no artinian ring-direct summands, $C$ exists by Lemma 1.4.

We call the ordered pair of rings $(\bar{\Lambda}, \bar{\Omega})$ an artinian pair. This is a slight generalization of what is called an artinian pair in [W' '89, GL '89, O '89], where $\bar{\Omega}$ is required to be a principal ideal ring. (This holds, in [W '89] and [GL '89] because $\Omega$ is a maximal order.) The decomposition (6.1.1) of $\Omega$ yields decompositions $C=\bigoplus_{k} C_{k}$ and $\bar{\Omega}=\bigoplus_{k} \bar{\Omega}_{k}$ where $\bar{\Omega}_{k}=\Omega_{k} / C_{k}$. Similarly, we let $\bar{B}_{k}=B_{k} / C_{k} B_{k}$, where $B_{k}$ is the unique indecomposable projective f.g. $\Omega_{k}$-module. (See Corollary 5.6.) The notation for maximal ideals $M_{k i} \subseteq \Omega_{k}$ and splitting numbers $s_{k i}$ retains its meaning, as defined in Notation 6.1, especially (6.1.5).

Caution. Throughout our discussion of artinian pairs, we use an overbar to denote some version of reduction modulo $C$ [e.g. $\bar{B}_{k}=B_{k} / C_{k} B_{k}$ ]. This differs from our earlier use of an overbar to denote reduction modulo the Jacobson radical, in the discussion of completions.

(6.18.1) Each $\bar{\Omega}_{k}=\bigoplus_{i} \bar{\Omega}_{k i}$ where each $\bar{\Omega}_{k i}$ is a matrix-local ring [i.e. $\bar{\Omega}_{k i} / J\left(\bar{\Omega}_{k i}\right)$ is a simple artinian ring] with maximal ideal $\bar{M}_{k i}$, where $M_{k i}$ is the maximal ideal of $\Omega_{k}$ defined in (6.1.5).

Proof. We have $\Omega / C \cong \hat{\Omega} / \hat{C}$, since $\Omega / C$ has finite length as an $R$-module [Proposition 3.8]; so the claim follows quickly from Lemma 6.5. But, since the spirit of artinian pairs is to avoid completions, we sketch an alternative proof. We can suppose that $\Omega=\Omega_{1}$ and $R=Z(\Omega)$. The commutative ring $\bar{R}=R /(C \cap R)$ is artinian, since it is an $R$-subalgebra of the $R$-module $\Omega / C$ of finite length. Therefore it is a direct sum of $m$ local rings, where $m$ is the number of maximal ideals of $\bar{R}$. This induces a decomposition of the $\bar{R}$-algebra $\bar{\Omega}$ into a direct sum of $m$ rings $\bar{\Omega}_{i}$. Thus $\bar{\Omega}$ has at least $m$ maximal ideals. But since the sets of maximal ideals of the rings $\Omega$ and $Z(\Omega)$ are in one-to-one correspondence via contraction [Lemma 5.3], the ring 
$\bar{\Omega}$ has the same number of maximal ideals as the ring $\bar{R}$. Therefore each $\bar{\Omega}_{i}$ must be matrix-local.

Since $C_{k} \subseteq J\left(\Omega_{k}\right)$, the simple (left) $\bar{\Omega}_{k}$-modules coincide with the simple $\Omega_{k^{-}}$ modules $S_{k i}$ [see (6.1.5)]. Therefore the following description of the projective $\bar{\Omega}$-modules is essentially the same as the description of the projective $\hat{\Omega}$-modules given in Theorem 6.6.

Theorem 6.19 (Projective $\bar{\Omega}$-modules). $\quad$ (i) For each $(k, i)$ there is a unique indecomposable f.g. projective (left) $\bar{\Omega}_{k}$-module $\bar{B}_{k i}$ that maps $\bar{\Omega}$-linearly onto $S_{k i}$. Every indecomposable f.g. projective $\bar{\Omega}$-module is isomorphic to some $\bar{B}_{k i}$.

(ii) $\bar{B}_{k} \cong \bigoplus_{i}\left(\bar{B}_{k i}\right)^{s_{k i}}$, and $\bar{\Omega}_{k} \cong \bigoplus_{i}\left(\bar{B}_{k i}\right)^{n_{k} s_{k i}}$, where $n_{k}$ is the matrix size of $\Omega_{k}$ [equivalently, of $\Gamma_{k}$ ]. [See (5.1.2) and (4.1.8).]

Proof. This and some other proofs that follow make use of projective covers of modules. See e.g. [LO, 8.8] for a review of their basic properties. Since the ring $\bar{\Omega}_{k}$ is artinian, each $S_{k i}$ has a projective cover $\bar{B}_{k i} \rightarrow S_{k i}$ as an $\bar{\Omega}_{k}$-module, and $\left\{\bar{B}_{k i}\right\}$ is a full set of nonisomorphic indecomposable projective left $\bar{\Omega}_{k}$-modules. Thus statement (i) holds.

To obtain (ii) note that by (6.1.5) there is a surjective $\bar{\Omega}$-module homomorphism: $\bar{B}_{k} \rightarrow \bigoplus_{i}\left(S_{k i}\right)^{s_{k i}}$ with kernel $J\left(\bar{\Omega}_{k}\right) \bar{B}_{k}$. This is a projective cover, as is $\bigoplus_{i}\left(\bar{B}_{k i}\right)^{s_{k i}} \rightarrow \bigoplus_{i}\left(S_{k i}\right)^{s_{k i}}$, the direct sum of the projective covers constructed earlier. Uniqueness of the projective cover now yields the first statement of (ii). The second statement of (ii) is obvious.

Notation 6.20. We define a $(\bar{\Lambda}, \bar{\Omega})$-module $[X \subseteq Y]$ to be a f.g. projective left $\bar{\Omega}$-module $Y$, together with a $\bar{\Lambda}$-submodule $X$ such that $\bar{\Omega} X=Y$. We say that $[X \subseteq Y] \cong\left[X^{\prime} \subseteq Y^{\prime}\right]$ if there is an $\bar{\Omega}$-isomorphism $Y \cong Y^{\prime}$ that carries $X$ onto $X^{\prime}$.

Every f.g. torsionfree $\Lambda$-module $A$ such that $\Omega A$ is $\Omega$-projective yields a $(\bar{\Lambda}, \bar{\Omega})$ module

$$
\Phi(A)=[A / C A \subseteq \Omega A / C A] .
$$

In order to state which $(\bar{\Lambda}, \bar{\Omega})$-modules arise from such an $A$, we define the array of ranks $\rho[X \subseteq Y]=\left(\left(\rho_{k i}\right)\right)$ to be the array of nonnegative integers $\rho_{k i}$ such that $Y \cong \bigoplus_{k i}\left(\bar{B}_{k i}\right)^{\rho_{k i}}$. See Remark 6.23, below, for the relationship between this and the "array of ranks" used in connection with the package deal theorem for completions. As with that definition, this array is not a matrix because it need not have the same number of entries in every row. Note that $\rho[X \subseteq Y]$ has one row for each prime ring-direct summand $\Omega_{k}$ of $\Omega$. In addition, $\rho[X \subseteq Y]$ is completely determined by $Y$.

Package Deal Theorem 6.21 (Artinian pairs). The following statements about a $(\bar{\Lambda}, \bar{\Omega})$-module $[X \subseteq Y]$ and a splitting order $\Omega \supseteq \Lambda$ are equivalent.

(i) $[X \subseteq Y] \cong \Phi(A)$ for some f.g. torsionfree $\Lambda$-module $A$ such that $\Omega A$ is $\Omega$ projective.

(ii) For each $k$, the array of ranks $\left(\left(\rho_{k i}\right)\right)=\rho[X \subseteq Y]$ satisfies

$$
\left(\rho_{k 1}, \rho_{k 2}, \ldots\right) \in \mathbb{N} \cdot\left(s_{k 1}, s_{k 2}, \ldots\right)
$$

where $\mathbb{N}$ denotes the nonnegative integers.

(iii) $Y \cong P / J(\Omega) \cdot P$ for some f.g. projective $\Omega$-module $P$. 
Moreover, $A$ is unique up to isomorphism when it exists.

Proof. (i) $\Rightarrow$ (ii). When $A$ exists, $Y \cong \Omega A / C A$. Since $\Omega A$ is $\Omega$-projective, we have $\Omega A \cong \bigoplus_{k}\left(B_{k}\right)^{m_{k}}$ for suitable nonnegative integers $m_{k}$. Theorem 6.19(ii) then shows that row $k$ of the resulting array of ranks is $m_{k}\left(s_{k 1}, s_{k 2}, \ldots\right)$, as desired.

(ii) $\Rightarrow$ (i). For each $k$ define $m_{k}$ by $\left(\rho_{k 1}, \rho_{k 2}, \ldots\right)=m_{k}\left(s_{k 1}, s_{k 2}, \ldots\right)$, and let $P=\bigoplus_{k}\left(B_{k}\right)^{m_{k}}$. Then $P / C P \cong Y$, and we can take $A$ to be the inverse image of $X$ in $P$.

(ii) $\Longleftrightarrow$ (iii). Statement (iii) is clearly just a way of restating (ii). It is included to emphasize the parallel between the present theorem and its counterpart for completions, Theorem 6.15.

Supplementary statement. Let $\Phi(A)=[X \subseteq Y]$ and $\Phi\left(A^{\prime}\right)=\left[X^{\prime} \subseteq Y^{\prime}\right]$, and suppose that $\Phi(A) \cong \Phi\left(A^{\prime}\right)$, that is, some $\bar{\Omega}$-isomorphism $\theta: Y \cong Y^{\prime}$ carries $X$ onto $X^{\prime}$. As already observed, the natural surjection $\Omega A \rightarrow Y$ is a projective cover. A similar statement applies to $A^{\prime}$. By uniqueness of the projective cover, $\theta$ can be lifted to an isomorphism $\Theta: \Omega A \cong \Omega A^{\prime}$. Since $\theta(X)=X^{\prime}$ we have $\Theta(A)=A^{\prime}$, as desired.

Proposition 6.22. The Krull-Schmidt theorem holds for modules over the artinian pair $(\bar{\Lambda}, \bar{\Omega})$.

Proof. We can identify a $(\bar{\Lambda}, \bar{\Omega})$-module $[X \subseteq Y]$ with the external direct sum $X \oplus Y$, its elements written in the form $\left[\begin{array}{l}x \\ y\end{array}\right]$. Then the collection of elements of the form $\left[\begin{array}{l}x \\ y\end{array}\right]$ can be regarded as a f.g. left module over the artinian ring $\left(\bar{\Lambda} \frac{0}{\Lambda}\right)$. Therefore the desired result follows from the Krull-Schmidt theorem for f.g. modules over artinian rings.

Remark 6.23. We have given two definitions of "array of ranks", one in connection with artinian pairs, and one in connection with completions [Notation 6.14]. The following fact shows that the two definitions define the same collection of arrays when they arise from a torsionfree $\Lambda$-module.

(6.23.1) Let $A$ be a torsionfree $\Lambda$-module such that $\Omega A$ is $\Omega$-projective, and $[A / C A \subseteq \Omega A / C A]=[X \subseteq Y]$ its associated $(\bar{\Lambda}, \bar{\Omega})$-module. Then $\rho[X \subseteq Y]$ $=\rho(\hat{A})$.

To prove this, first observe that $\Omega A / C A \cong \hat{\Omega} \hat{A} / \hat{C} \hat{A}$, since the left-hand side is an $R$-module of finite length [Lemma 1.6]. Then note that, since $C \subseteq J(\Omega)$, the natural maps $\Omega A \rightarrow \Omega A / C A$ and $\hat{\Omega} A \rightarrow \hat{\Omega} A / \hat{C} \hat{A}$ are projective covers, with respect to $\Omega$ and $\hat{\Omega}$, respectively. Uniqueness of projective covers, together with the structure of projective $\bar{\Omega}$ and $\hat{\Omega}$-modules given in Theorems 6.19 and 6.6 , then finishes the proof.

Acknowledgements. We wish to thank Robert Guralnick, William Heinzer, Lee Klingler, and Nikolaus Vonessen for their valuable suggestions which helped us avoid the standard hypothesis that orders are contained in maximal orders. Without these suggestions our Krull-Schmidt results in [LO] would not have been as complete as they now are.

\section{REFERENCES}

[B '72] N. Bourbaki, Commutative Algebra, Hermann, Paris, 1972. MR 50:12997

[CR '81] C. W. Curtis and I Reiner, Methods of Representation Theory, with Applications to Finite Groups and Orders, vol. 1, Wiley-Interscience, New York, 1981. MR 82i:20001 
[G '65] A. Grothendieck, "Éléments de Géométrie Algébrique, IV", in Publ. Math. IHES 24 (1965). MR 33:7330

[GL '88] R. M. Guralnick and L. S. Levy, "Presentations of modules when ideals need not be principal", Ill. J. Math. 32 (1988), 593-653. MR 90c:16004

[GL '89] R. M. Guralnick, and L. S. Levy, "Cancellation and direct summands in dimension 1", J. Alg. 142 (1991), 310-347. MR 92h:13012

[H '61] A. Heller, "On group representations over a valuation ring", Proc. Nat. Acad. of Sci. 47 (1961), 1194-1197. MR 23:A2468

[HL '87] J. Haefner and L. S. Levy, "Commutative orders whose lattices are direct sums of ideals", J. Pure and Appl. Algebra, 50 (1988), 1-20. MR 89e:13005

[H '73] M. Hochster, "Non-openness of loci in noetherian rings", Duke Math J. 40 (1973), 215219. MR 47:215

[J '56] N. Jacobson, Structure of Rings, Amer. Math. Soc. Colloq. Publ. 37, New York, 1956. MR 18:373d

[K '70] I. Kaplansky, Commutative Rings, Allyn and Bacon, Boston, 1970. MR 40:7234

[Kt '86] D. Katz, "On the number of minimal prime ideals in the completion of a local domain", Rocky Mountain J. Math. 16 (1986), 575-578. MR 87k:13014

[L '89] L. S. Levy, "Projectives of large uniform-rank, in Krull dimension 1", Bulletin London Math. Soc. 21 (1989), 57-64. MR 89k:16048

[LO] L. S. Levy and C. J. Odenthal, "Krull-Schmidt Theorems in Dimension 1", Trans. Amer. Math. Soc. 348 (1996), 3391-3455.

[MR '87] J. C. McConnell and J. C. Robson, Noncommutative Noetherian Rings, WileyInterscience, Chichester, 1987. MR 89j:16023

[Mtl '73] E. Matlis, 1-Dimensional Cohen-Macaulay Rings, Lecture Notes in Math 327, Springer, New York, 1973. MR 50:9859

[Mts '80] H. Matsumura, Commutative Algebra, second edition, Bemjamin-Cummings, Reading, Mass., 1980. MR 82i:13003

[N '62] M. Nagata, Local Rings, Interscience, New York, 1962. MR 27:5790

[O '89] C. J. Odenthal, "Presentations over HNP rings with enough invertible ideals and torsionfree cancellation over neoclassical orders", J. Algebra 127 (1989), 290-319. MR 90m:16008

[R '75] I. Reiner, Maximal Orders, Academic Press, New York, 1975. MR 52:13910

[RS '74] J. C. Robson and L. Small, "Hereditary prime P.I. rings are classical orders", J. London Math. Soc. (2) 8 (1974), 499-503. MR 50:2236

[W '89] R. Wiegand, "Noetherian rings of bounded representation type", in Proceedings of Microprogram on Commutative Algebra, MSRI, Berkeley, 1987 (Springer, New York, 1989), 497-516. MR 90i: 13010

Department of Mathematics, University of Wisconsin, Madison, Wisconsin 53706-1388 E-mail address: levy@math.wisc.edu

Department of Mathematics, University of Toledo, Toledo, Ohio 43606-3390

E-mail address: codentha@math.utoledo.edu 